EUROPEAN ORGANIZATION FOR NUCLEAR RESEARCH

CERN-PPE/95-140

September 12, 1995

\title{
Tau hadronic branching ratios
}

\author{
The ALEPH Collaboration*
}

\begin{abstract}
From 64492 selected $\tau$-pair events, produced at the $Z^{0}$ resonance, the measurement of the tau decays into hadrons from a global analysis using 1991, 1992 and 1993 ALEPH data is presented. Special emphasis is given to the reconstruction of photons and $\pi^{0}$ s, and the removal of fake photons. A detailed study of the systematics entering the $\pi^{0}$ reconstruction is also given. A complete and consistent set of tau hadronic branching ratios is presented for 18 exclusive modes. Most measurements are more precise than the present world average. The new level of precision reached allows a stringent test of $\tau-\mu$ universality in hadronic decays, $g_{\tau} / g_{\mu}=1.0013 \pm 0.0095$, and the first measurement of the vector and axial-vector contributions to the non-strange hadronic $\tau$ decay width: $R_{\tau, V}=1.788 \pm 0.025$ and $R_{\tau, A}=1.694 \pm 0.027$. The ratio $\left(R_{\tau, V}-R_{\tau, A}\right) /\left(R_{\tau, V}+\right.$ $\left.R_{\tau, A}\right)$, equal to $(2.7 \pm 1.3) \%$, is a measure of the importance of QCD non-perturbative contributions to the hadronic $\tau$ decay width.
\end{abstract}

(Submitted to Zeitschrift für Physik)

*) See next pages for the list of authors 


\section{The ALEPH Collaboration}

D. Buskulic, D. Casper, I. De Bonis, D. Decamp, P. Ghez, C. Goy, J.-P. Lees, A. Lucotte, M.-N. Minard, P. Odier, B. Pietrzyk

Laboratoire de Physique des Particules (LAPP), IN ${ }^{2} P^{3}$-CNRS, 74019 Annecy-le-Vieux Cedex, France

F. Ariztizabal, M. Chmeissani, J.M. Crespo, I. Efthymiopoulos, E. Fernandez, M. Fernandez-Bosman, V. Gaitan, Ll. Garrido, ${ }^{15}$ M. Martinez, S. Orteu, A. Pacheco, C. Padilla, F. Palla, A. Pascual, J.A. Perlas, F. Sanchez, F. Teubert

Institut de Fisica d'Altes Energies, Universitat Autonoma de Barcelona, 08193 Bellaterra (Barcelona), Spain $^{7}$

A. Colaleo, D. Creanza, M. de Palma, A. Farilla, G. Gelao, M. Girone, G. Iaselli, G. Maggi, ${ }^{3}$ M. Maggi, N. Marinelli, S. Natali, S. Nuzzo, A. Ranieri, G. Raso, F. Romano, F. Ruggieri, G. Selvaggi, L. Silvestris, P. Tempesta, G. Zito

Dipartimento di Fisica, INFN Sezione di Bari, 70126 Bari, Italy

X. Huang, J. Lin, Q. Ouyang, T. Wang, Y. Xie, R. Xu, S. Xue, J. Zhang, L. Zhang, W. Zhao

Institute of High-Energy Physics, Academia Sinica, Beijing, The People's Republic of China ${ }^{8}$

G. Bonvicini, M. Cattaneo, P. Comas, P. Coyle, H. Drevermann, A. Engelhardt, R.W. Forty, M. Frank, R. Hagelberg, J. Harvey, R. Jacobsen, ${ }^{24}$ P. Janot, B. Jost, E. Kneringer, J. Knobloch, I. Lehraus, C. Markou, ${ }^{23}$

E.B. Martin, P. Mato, A. Minten, R. Miquel, T. Oest, P. Palazzi, J.R. Pater, ${ }^{27}$ J.-F. Pusztaszeri, F. Ranjard, P. Rensing, L. Rolandi, D. Schlatter, M. Schmelling, O. Schneider, W. Tejessy, I.R. Tomalin, A. Venturi, H. Wachsmuth, W. Wiedenmann, T. Wildish, W. Witzeling, J. Wotschack

European Laboratory for Particle Physics (CERN), 1211 Geneva 23, Switzerland

Z. Ajaltouni, M. Bardadin-Otwinowska, ${ }^{2}$ A. Barres, C. Boyer, A. Falvard, P. Gay, C. Guicheney, P. Henrard, J. Jousset, B. Michel, S. Monteil, J-C. Montret, D. Pallin, P. Perret, F. Podlyski, J. Proriol, J.-M. Rossignol, F. Saadi

Laboratoire de Physique Corpusculaire, Université Blaise Pascal, IN $N^{2} P^{3}$-CNRS, Clermont-Ferrand, 63177 Aubière, France

T. Fearnley, J.B. Hansen, J.D. Hansen, J.R. Hansen, P.H. Hansen, B.S. Nilsson

Niels Bohr Institute, 2100 Copenhagen, Denmark ${ }^{9}$

A. Kyriakis, E. Simopoulou, I. Siotis, A. Vayaki, K. Zachariadou

Nuclear Research Center Demokritos (NRCD), Athens, Greece

A. Blondel, ${ }^{21}$ G. Bonneaud, J.C. Brient, P. Bourdon, L. Passalacqua, A. Rougé, M. Rumpf, R. Tanaka, A. Valassi, ${ }^{6}$ M. Verderi, H. Videau

Laboratoire de Physique Nucléaire et des Hautes Energies, Ecole Polytechnique, IN $N^{2} P^{3}$-CNRS, 91128

Palaiseau Cedex, France

D.J. Candlin, M.I. Parsons

Department of Physics, University of Edinburgh, Edinburgh EH9 3JZ, United Kingdom ${ }^{10}$

E. Focardi, G. Parrini

Dipartimento di Fisica, Università di Firenze, INFN Sezione di Firenze, 50125 Firenze, Italy

M. Corden, M. Delfino, ${ }^{12}$ C. Georgiopoulos, D.E. Jaffe 
Supercomputer Computations Research Institute, Florida State University, Tallahassee, FL 32306$4052, \operatorname{USA}^{13,14}$

A. Antonelli, G. Bencivenni, G. Bologna, ${ }^{4}$ F. Bossi, P. Campana, G. Capon, V. Chiarella, G. Felici, P. Laurelli, G. Mannocchi, ${ }^{5}$ F. Murtas, G.P. Murtas, M. Pepe-Altarelli

Laboratori Nazionali dell'INFN (LNF-INFN), 00044 Frascati, Italy

S.J. Dorris, A.W. Halley, I. ten Have, ${ }^{6}$ I.G. Knowles, J.G. Lynch, W.T. Morton, V. O'Shea, C. Raine, P. Reeves, J.M. Scarr, K. Smith, M.G. Smith, A.S. Thompson, F. Thomson, S. Thorn, R.M. Turnbull

Department of Physics and Astronomy, University of Glasgow, Glasgow G12 8QQ,United Kingdom ${ }^{10}$

U. Becker, O. Braun, C. Geweniger, G. Graefe, P. Hanke, V. Hepp, E.E. Kluge, A. Putzer, B. Rensch, M. Schmidt, J. Sommer, H. Stenzel, K. Tittel, S. Werner, M. Wunsch

Institut für Hochenergiephysik, Universität Heidelberg, 69120 Heidelberg, Fed. Rep. of Germany ${ }^{16}$

R. Beuselinck, D.M. Binnie, W. Cameron, D.J. Colling, P.J. Dornan, N. Konstantinidis, L. Moneta, A. Moutoussi, J. Nash, G. San Martin, J.K. Sedgbeer, A.M. Stacey

Department of Physics, Imperial College, London SW7 2BZ, United Kingdom ${ }^{10}$

G. Dissertori, P. Girtler, D. Kuhn, G. Rudolph

Institut für Experimentalphysik, Universität Innsbruck, 6020 Innsbruck, Austria $^{18}$

C.K. Bowdery, T.J. Brodbeck, P. Colrain, G. Crawford, A.J. Finch, F. Foster, G. Hughes, T. Sloan, E.P. Whelan, M.I. Williams

Department of Physics, University of Lancaster, Lancaster LA1 4YB, United Kingdom ${ }^{10}$

A. Galla, A.M. Greene, K. Kleinknecht, G. Quast, J. Raab, B. Renk, H.-G. Sander, R. Wanke, P. van Gemmeren C. Zeitnitz

Institut für Physik, Universität Mainz, 55099 Mainz, Fed. Rep. of Germany ${ }^{16}$

J.J. Aubert, A.M. Bencheikh, C. Benchouk, A. Bonissent, ${ }^{21}$ G. Bujosa, D. Calvet, J. Carr, C. Diaconu, F. Etienne, M. Thulasidas, D. Nicod, P. Payre, D. Rousseau, M. Talby

Centre de Physique des Particules, Faculté des Sciences de Luminy, IN ${ }^{2} P^{3}$-CNRS, 13288 Marseille,

France

I. Abt, R. Assmann, C. Bauer, W. Blum, D. Brown, ${ }^{24}$ H. Dietl, F. Dydak,${ }^{21}$ G. Ganis, C. Gotzhein, K. Jakobs, H. Kroha, G. Lütjens, G. Lutz, W. Männer, H.-G. Moser, R. Richter, A. Rosado-Schlosser, S. Schael, R. Settles, H. Seywerd, R. St. Denis, G. Wolf

Max-Planck-Institut für Physik, Werner-Heisenberg-Institut, 80805 München, Fed. Rep. of Germany ${ }^{16}$

R. Alemany, J. Boucrot, O. Callot, A. Cordier, F. Courault, M. Davier, L. Duflot, J.-F. Grivaz, Ph. Heusse, M. Jacquet, D.W. Kim ${ }^{19}$ F. Le Diberder, J. Lefrançois, A.-M. Lutz, G. Musolino, I. Nikolic, H.J. Park, I.C. Park, M.-H. Schune, S. Simion, J.-J. Veillet, I. Videau

Laboratoire de l'Accélérateur Linéaire, Université de Paris-Sud, IN $\mathrm{N}^{2} \mathrm{P}^{3}-\mathrm{CNRS}, 91405$ Orsay Cedex, France

D. Abbaneo, P. Azzurri, G. Bagliesi, G. Batignani, S. Bettarini, C. Bozzi, G. Calderini, M. Carpinelli, M.A. Ciocci, V. Ciulli, R. Dell'Orso, R. Fantechi, I. Ferrante, L. Foà, ${ }^{1}$ F. Forti, A. Giassi, M.A. Giorgi, A. Gregorio, F. Ligabue, A. Lusiani, P.S. Marrocchesi, A. Messineo, G. Rizzo, G. Sanguinetti, A. Sciabà, P. Spagnolo, J. Steinberger, R. Tenchini, G. Tonelli, ${ }^{26}$ G. Triggiani, C. Vannini, P.G. Verdini, J. Walsh

Dipartimento di Fisica dell'Università, INFN Sezione di Pisa, e Scuola Normale Superiore, 56010 Pisa, Italy

A.P. Betteridge, G.A. Blair, L.M. Bryant, F. Cerutti, Y. Gao, M.G. Green, D.L. Johnson, T. Medcalf, Ll.M. Mir, P. Perrodo, J.A. Strong

Department of Physics, Royal Holloway \& Bedford New College, University of London, Surrey TW20 OEX, United Kingdom ${ }^{10}$

V. Bertin, D.R. Botterill, R.W. Clifft, T.R. Edgecock, S. Haywood, M. Edwards, P. Maley, P.R. Norton, J.C. Thompson

Particle Physics Dept., Rutherford Appleton Laboratory, Chilton, Didcot, Oxon OX11 OQX, United Kingdom ${ }^{10}$ 
B. Bloch-Devaux, P. Colas, S. Emery, W. Kozanecki, E. Lançon, M.C. Lemaire, E. Locci, B. Marx, P. Perez, J. Rander, J.-F. Renardy, A. Roussarie, J.-P. Schuller, J. Schwindling, A. Trabelsi, B. Vallage

CEA, DAPNIA/Service de Physique des Particules, CE-Saclay, 91191 Gif-sur-Yvette Cedex, France ${ }^{17}$

R.P. Johnson, H.Y. Kim, A.M. Litke, M.A. McNeil, G. Taylor

Institute for Particle Physics, University of California at Santa Cruz, Santa Cruz, CA 95064, USA ${ }^{22}$

A. Beddall, C.N. Booth, R. Boswell, S. Cartwright, F. Combley, I. Dawson, A. Koksal, M. Letho, W.M. Newton, C. Rankin, L.F. Thompson

Department of Physics, University of Sheffield, Sheffield S3 7RH, United Kingdom ${ }^{10}$

A. Böhrer, S. Brandt, G. Cowan, E. Feigl, C. Grupen, G. Lutters, J. Minguet-Rodriguez, F. Rivera, ${ }^{25}$ P. Saraiva, L. Smolik, F. Stephan,

Fachbereich Physik, Universität Siegen, 57068 Siegen, Fed. Rep. of Germany ${ }^{16}$

M. Apollonio, L. Bosisio, R. Della Marina, G. Giannini, B. Gobbo, F. Ragusa ${ }^{20}$

Dipartimento di Fisica, Università di Trieste e INFN Sezione di Trieste, 34127 Trieste, Italy

J. Rothberg, S. Wasserbaech

Experimental Elementary Particle Physics, University of Washington, WA 98195 Seattle, U.S.A.

S.R. Armstrong, L. Bellantoni, ${ }^{30}$ P. Elmer, Z. Feng, D.P.S. Ferguson, Y.S. Gao, S. González, J. Grahl, J.L. Harton, ${ }^{28}$ O.J. Hayes, H. Hu, P.A. McNamara III, J.M. Nachtman, W. Orejudos, Y.B. Pan, Y. Saadi, M. Schmitt, I.J. Scott, V. Sharma, ${ }^{29}$ J.D. Turk, A.M. Walsh, Sau Lan Wu, X. Wu, J.M. Yamartino, M. Zheng, G. Zobernig

Department of Physics, University of Wisconsin, Madison, WI 53706, USA ${ }^{11}$

\footnotetext{
${ }^{1}$ Now at CERN, 1211 Geneva 23, Switzerland.

${ }^{2}$ Deceased.

${ }^{3}$ Now at Dipartimento di Fisica, Università di Lecce, 73100 Lecce, Italy.

${ }^{4}$ Also Istituto di Fisica Generale, Università di Torino, Torino, Italy.

${ }^{5}$ Also Istituto di Cosmo-Geofisica del C.N.R., Torino, Italy.

${ }^{6}$ Supported by the Commission of the European Communities, contract ERBCHBICT 941234.

${ }^{7}$ Supported by CICYT, Spain.

${ }^{8}$ Supported by the National Science Foundation of China.

${ }^{9}$ Supported by the Danish Natural Science Research Council.

${ }^{10}$ Supported by the UK Particle Physics and Astronomy Research Council.

${ }^{11}$ Supported by the US Department of Energy, grant DE-FG0295-ER40896.

${ }^{12}$ On leave from Universitat Autonoma de Barcelona, Barcelona, Spain.

${ }^{13}$ Supported by the US Department of Energy, contract DE-FG05-92ER40742.

${ }^{14}$ Supported by the US Department of Energy, contract DE-FC05-85ER250000.

${ }^{15}$ Permanent address: Universitat de Barcelona, 08208 Barcelona, Spain.

${ }^{16}$ Supported by the Bundesministerium für Forschung und Technologie, Fed. Rep. of Germany.

${ }^{17}$ Supported by the Direction des Sciences de la Matière, C.E.A.

${ }^{18}$ Supported by Fonds zur Förderung der wissenschaftlichen Forschung, Austria.

${ }^{19}$ Permanent address: Kangnung National University, Kangnung, Korea.

${ }^{20}$ Now at Dipartimento di Fisica, Università di Milano, Milano, Italy.

${ }^{21}$ Also at CERN, 1211 Geneva 23, Switzerland.

${ }^{22}$ Supported by the US Department of Energy, grant DE-FG03-92ER40689.

${ }^{23}$ Now at University of Athens, 157-71 Athens, Greece.

${ }^{24}$ Now at Lawrence Berkeley Laboratory, Berkeley, CA 94720, USA.

${ }^{25}$ Partially supported by Colciencias, Colombia.

${ }^{26}$ Also at Istituto di Matematica e Fisica, Università di Sassari, Sassari, Italy.

${ }^{27}$ Now at Schuster Laboratory, University of Manchester, Manchester M13 9PL, UK.

${ }^{28}$ Now at Colorado State University, Fort Collins, CO 80523, USA.

${ }^{29}$ Now at University of California at San Diego, La Jolla, CA 92093, USA.

${ }^{30}$ Now at Fermi National Accelerator Laboratory, Batavia, IL 60510, USA.
} 


\section{Contents}

1 Introduction $\quad 1$

2 The ALEPH detector 2

3 Tau event selection $\quad 3$

4 Particle Identification 4

5 Photons $\quad 7$

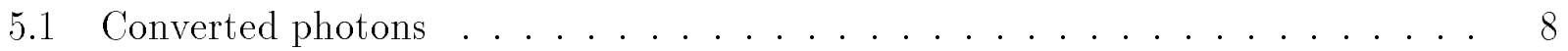

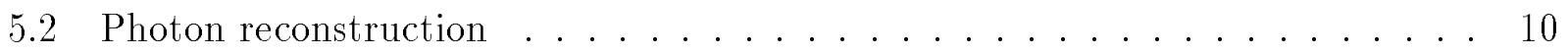

5.3 Likelihood method . . . . . . . . . . . . . . . . 11

$6 \pi^{0}$ reconstruction $\quad 14$

6.1 Resolved $\pi^{0}{ }_{\mathrm{s}} \ldots \ldots \ldots \ldots \ldots \ldots \ldots \ldots$

6.2 Unresolved $\boldsymbol{\pi}^{0} \mathrm{~s} \ldots \ldots \ldots \ldots \ldots \ldots \ldots$

6.3 Residual single photons . . . . . . . . . . . . . . . . . . 19

6.4 Summary on $\pi^{0}$ reconstruction ........................ 23

7 Tau decay classification $\quad 26$

8 Extraction of branching ratios $\quad 33$

9 Systematic uncertainties $\quad 34$

9.1 Photon and $\pi^{0}$ reconstruction . . . . . . . . . . . . . 34

9.2 Tau selection and particle identification . . . . . . . . . . . . . . . 38

9.3 Tracking and secondary interactions . . . . . . . . . . . . . . 40

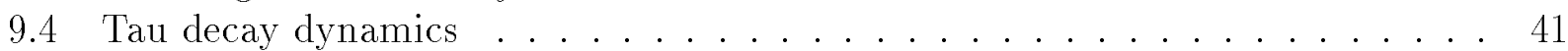

9.5 Summary on systematic uncertainties . . . . . . . . . . . . . 42

10 Quasi-exclusive and exclusive results 43

11 Update on $\tau$ decays to charged and neutral kaons $\quad 48$

12 Corrected branching ratio results $\quad 52$

13 Discussion of the results $\quad 53$

14 Conclusions $\quad 63$ 
. 


\section{Introduction}

The $\tau$ leptons produced in $Z^{0}$ decays provide a powerful tool to study in detail the neutral and charged weak currents [1]. From the $\tau$ decays several precise lepton universality tests can be performed and the hadronic weak current can be investigated directly. Hadronic $\tau$ decays have proved to be a useful QCD laboratory, justifying detailed studies of the different final states. Finally, the measurement of the $\tau$ polarization in $Z^{0}$ decays requires an accurate knowledge of the $\tau$ properties and its decay dynamics. Past experiments have produced some controversial results regarding the decay rate of the dominant channels for one- and threeprong decays, leading to a certain deficit between the inclusive one-prong decay fraction and the sum of the exclusive one-prong decay rates, the so-called one-prong problem.

A systematic effort to resolve these difficulties was undertaken by the CELLO [2] and ALEPH [3] collaborations, by adopting a global method for measuring the $\tau$ branching ratios. These analyses resulted in a picture of $\tau$ decays consistent with the Standard Model. The global analysis presented in this paper follows the same approach, taking advantage of a large data sample collected between 1991 and 1993 and of improvements in the $\pi^{0}$ reconstruction.

Only the hadronic modes from the global branching ratio analysis are discussed in this publication. Given that around $70 \%$ of the hadronic modes have at least one $\pi^{0}$ in the final state, special emphasis is given to the reconstruction of photons and $\pi^{0}$ 's, the fake photon rejection, and the corresponding systematic effects.

The electronic and muonic branching ratios are determined both by the global approach and by a specific analysis described in a separate paper [4]. Both methods are in fact formally equivalent, but in practice they have used slightly different selection procedures and they are not based on exactly the same data sample. Since the analysis of the leptonic branching ratios involves an accurate study of particle identification, its detailed description is given in reference [4], while this paper concentrates on the $\pi^{0}$ reconstruction.

An update with the 1993 data of the $\tau$ branching ratios involving kaons is also given, following the same method as in the previous ALEPH publications [5, 6]. This information is

used to extract branching ratios of exclusive modes taking the kaon production properly into account.

The new level of precision reached measuring the $\tau$ hadronic branching ratios, due to the increased statistics and to the new algorithm developed for $\pi^{0}$ and fake photon identification, allows more stringent tests of the Standard Model through isospin invariance of the quark currents (generally expressed as the Conserved Vector Current hypothesis), together with an additional test of $\tau-\mu$ universality using the $\pi$ and $K$ decay rates. Moreover, a consistent study of the vector and axial vector contributions to the total $\tau$ hadronic width is also possible given the complete classification of $\tau$ decays provided in this paper.

The organization of this paper is as follows. After $\tau$ selection and particle identification, 
the photon and $\pi^{0}$ reconstruction are presented. Then, the $\tau$ decay classification and the branching ratio extraction are given. The different systematic components affecting the branching ratios are discussed in detail. The results from the constrained global analysis are given. Finally, the complete set of branching ratios obtained allows tests of the $\tau$ decay description in the Standard Model.

\section{The ALEPH detector}

A detailed description of the ALEPH detector can be found elsewhere [7, 8]. The features relevant to this analysis are briefly mentioned here.

Charged particles are measured by means of three detectors. The closest detector to the interaction region is a silicon vertex detector (VDET), which consists of two concentric barrels of microstrip silicon detectors. An inner tracking chamber (ITC), with eight drift chamber layers, surrounds the VDET detector. The ITC is followed by a time projection chamber (TPC), a cylindrical three-dimensional imaging drift chamber, providing up to 21 space points for charged particles, and up to 338 measurements of the ionization loss. Combining the coordinate measurements of these detectors, a momentum resolution $\delta p_{T} / p_{T}^{2}=6 \cdot 10^{-4} \oplus 5 \cdot 10^{-3} / p_{T}$ (with $p_{T}$ in $\mathrm{GeV} / \mathrm{c}^{-1}$ ) is achieved in the presence of a 1.5 Tesla magnetic field.

The electromagnetic calorimeter (ECAL), located inside the coil, is constructed from 45 layers of lead interleaved with proportional wire chambers. The position and energy of electromagnetic showers are measured using cathode pads subtending a solid angle of $0.9^{\circ} \times 0.9^{\circ}$ and connected internally to form projective towers. Each tower is read out in three segments with a depth of 4,9 and 9 radiation lengths, yielding an energy resolution $\delta E / E=18 \% / \sqrt{E}+0.9 \%$ (with $\mathrm{E}$ in $\mathrm{GeV}$ ). The inactive zones of this detector represent $2 \%$ in the barrel and $6 \%$ in the endcaps. The analysis of the hadronic $\tau$ decays presented in this paper benefits from the fine granularity and from the longitudinal segmentation of the calorimeter, which play a crucial role in the photon and $\pi^{0}$ reconstruction, and in the identification of fake photons.

The hadron calorimeter (HCAL) is composed of the iron of the magnet return yoke interleaved with 23 layers of streamer tubes and has a projective tower cathode pad readout of hadronic energy with a resolution of about $85 \% / \sqrt{E}$. Outside this calorimeter structure are located two additional double layers of streamer tubes, providing three-dimensional coordinates for particles passing through the HCAL.

The trigger efficiency for $\tau$ pair events is measured by comparing redundant and independent triggers involving the tracking detectors and the main calorimeters. The measured trigger efficiency is better than $99.99 \%$ within the selection cuts of this analysis. 
Tau-pair events are simulated by means of a Monte Carlo program which includes initial state radiation computed up to order $\alpha^{2}$ and exponentiated, and final state radiative corrections to order $\alpha[9]$. The simulation of the subsequent $\tau$ decays also includes single photon radiation for the decays with up to three hadrons in the final state. The longitudinal spin correlation is taken into account [10]. This simulation, with the detector acceptance and resolution effects, is used to evaluate the corresponding relative efficiencies and backgrounds. It also includes the tracking, the secondary interactions of hadrons, bremsstrahlung and conversions. Electromagnetic showers are generated in ECAL according to parameterizations obtained from test beam data [7].

\section{Tau event selection}

Tau pair candidates are selected by retaining low-multiplicity events coming mainly from lepton-pair decays of the $Z^{0}$, starting with a $\tau$ pre-selection as given in reference [11], except for the modifications regarding the cuts against $Z^{0} \rightarrow q \bar{q}$ background. A description of the additional cuts applied to suppress the Bhabha and dimuon events, $\gamma \gamma$ processes and cosmic rays is found in reference [4]. In this section, only the background reduction of $q \bar{q}$ events will be described with some detail, since it is the most severe non- $\tau$ background for the branching ratios considered in this analysis.

Each event is divided in two hemispheres with the plane perpendicular to the thrust axis, and their respective jets are obtained with an energy-flow algorithm [8] which calculates all the visible energy avoiding double-counting between the TPC and the calorimeters information. In this procedure the energy of the electromagnetic and hadronic neutral calorimeter objects (clusters not associated to charged tracks) must exceed 1 and $1.5 \mathrm{GeV}$, respectively, in order to be used in the event selection. This minimal energy requirement for the neutral calorimeter objects is introduced in order to be less sensitive to shower fluctuations and to reduce systematic effects. The $\tau$ pair events are required to have fewer than nine charged tracks coming from the interaction region. A charged track must have at least four TPC coordinates, its impact parameter in the plane perpendicular to the beam direction $\left(d_{0}\right)$ must be smaller than $2 \mathrm{~cm}$ and the distance from the interaction region along the beam axis must lie within $\pm 10 \mathrm{~cm}$.

Events where at least one hemisphere has only one charged track or an invariant mass smaller than $0.8 \mathrm{GeV} / \mathrm{c}^{2}$ (" $\tau$-like hemispheres") are preserved from the cuts designed to remove hadronic $Z$ decays. Hadronic event rejection is obtained by imposing that the product of the number of charged and neutral objects in each hemisphere $\left(n_{1} \cdot n_{2}\right)$ is smaller than 40 and the sum of the maximum angle between two tracks in each hemisphere $\left(\theta_{1}^{o p}+\theta_{2}^{o p}\right)$ is smaller than $0.25 \mathrm{rad}$. The values used for the cuts are slightly different in a first version of the $\tau$ selection used in reference [4]. The new version of the selection used in the present paper, is introduced in order to reduce systematic effects in the hadronic channels. Given the importance of this 
rejection for the hadronic $\tau$ decays selection, the corresponding efficiency is measured from data and Monte Carlo samples. Taking advantage of the fact that the two jets are essentially uncorrelated, unbiased samples of hadronic jets are constructed both in data and Monte Carlo (using the hadronic hemispheres opposite to the above defined $\tau$-like hemispheres), allowing a direct measurement of the hadronic cut efficiency. After a correction for the small jet correlation obtained from the Monte Carlo [4], the values $94.41 \pm 0.13 \%$ and $94.65 \pm 0.08 \%$ are obtained for data and Monte Carlo, respectively, in fair agreement. After taking into account all the cuts in the $\tau$ event selection affecting hadronic final states, the measured correction to the Monte Carlo efficiency in the hadron-hadron topology is found to be

$$
\frac{\Delta \varepsilon_{h h}}{\varepsilon_{h h}}=(-1.5 \pm 0.8) \cdot 10^{-3}
$$

This result shows the robustness of the hadronic cuts against the quality of the detector simulation. Nevertheless a correction is applied in the analysis to take into account the possible small discrepancy. Similar studies are carried out for the $\epsilon$-X and $\mu$-X final states. They are described in reference [4]. Good agreement is observed between the selection efficiencies measured from data and those using simulation. Thus, it is concluded that the efficiencies of $\tau$ event selection used in this analysis are correctly predicted by the simulation at the $10^{-3}$ level. The resulting systematic effects in the branching ratio analysis are discussed in Section 9.2.

The remaining $q \bar{q}$ background is estimated from the Monte Carlo samples to be $(0.31 \pm$ $0.09) \%$. A consistency check is obtained by comparing the $n_{1} \cdot n_{2}$ versus $\theta_{1}^{o p}+\theta_{2}^{o p}$ distribution between data and Monte Carlo, where no discrepancy is found. In addition, a normalization constant for the $q \bar{q}$ background can be fitted to data in the aforementioned two-dimensional distribution using its shape as predicted by the Monte Carlo. The result of the fit confirms the absolute Monte Carlo estimate at the $10 \%$ level. Finally, to illustrate the separation of $\tau$ pairs and $Z^{0} \rightarrow q \bar{q}$ events in a simple way, the distribution for the product of the $\theta_{1}^{o p}+\theta_{2}^{o p}$ and $n_{1} \cdot n_{2}$ variables is plotted in Figure 1 for data and Monte Carlo, and a good agreement is observed.

The study presented here is based on $67 \mathrm{pb}^{-1}$ of integrated luminosity collected with the ALEPH detector between 1991 and 1993 around the $Z^{0}$ resonance, of which $68 \%$ is taken

at the $Z^{0}$ peak energy. Table 1 summarizes the values obtained for the efficiency and the contaminations from all the involved processes. The total non- $\tau$ background contribution amounts to $(0.85 \pm 0.10) \%$ for the 64492 selected $\tau$ pair events in the data sample.

\section{Particle Identification}

The global branching ratio analysis presented in this paper uses an improved version of the likelihood method for charged particle identification originally described in reference [3] and 


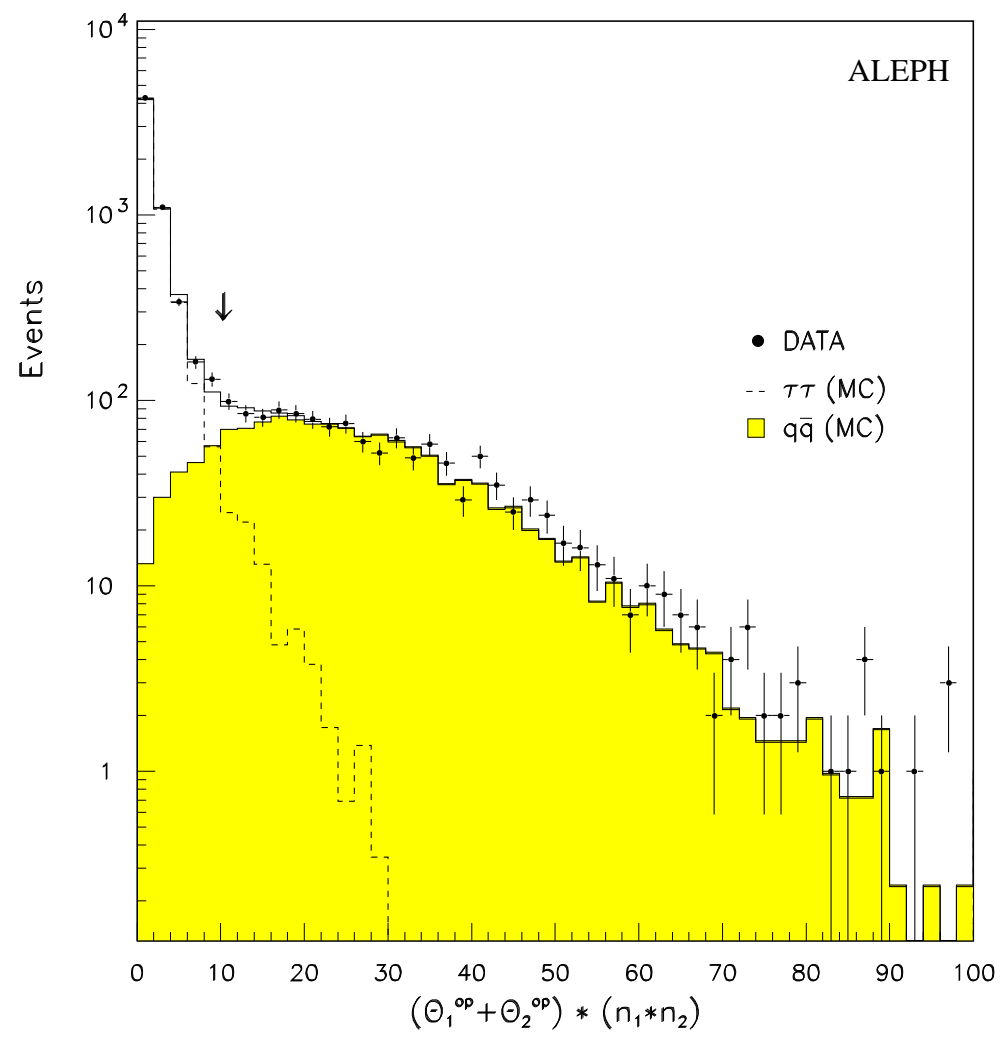

Figure 1: The distribution for the product of $n_{1} \cdot n_{2}$ and $\theta_{1}^{o p}+\theta_{2}^{o p}$ variables is plotted. The points with error bars show the data and the solid histogram the Monte Carlo expectation. The shaded histogram shows the contribution for the $q \bar{q}$ events and the arrow indicates the location of the product of the individual cut values for $n_{1} \cdot n_{2}$ and $\theta_{1}^{o p}+\theta_{2}^{o p}$.

explained in detail in [4].

Charged particle identification is required at two different stages. First, electrons are identified among charged tracks in any given topology in order to reconstruct the converted photons. Secondly, once converted photons are identified, global particle identification is used for the remaining tracks to separate electrons, muons and hadrons in one-prong hemispheres.

The particle identification is based on the following information:

- $\frac{d E}{d x}$ in the TPC,

- longitudinal and transverse shower profile in ECAL near the extrapolated track,

- energy and average shower width in HCAL, together with the number of fired planes in the last 10 planes of HCAL and hits in the muon chambers. 


\begin{tabular}{|c|c|c|}
\hline Physics processes & Efficiency (\%) & Contamination (\%) \\
\hline \hline$Z^{0} \rightarrow \tau^{+} \tau^{-}$ & $78.84 \pm 0.13$ & \\
\hline Bhabha & & $0.15 \pm 0.03$ \\
\hline$Z^{0} \rightarrow \mu^{+} \mu^{-}$ & & $0.07 \pm 0.02$ \\
\hline$\gamma \gamma \rightarrow e^{+} e^{-}$ & & $0.07 \pm 0.02$ \\
\hline$\gamma \gamma \rightarrow \mu^{+} \mu^{-}$ & & $0.08 \pm 0.02$ \\
\hline four-fermion & & $0.14 \pm 0.02$ \\
\hline cosmic rays & & $0.02 \pm 0.01$ \\
\hline$Z^{0} \rightarrow q \bar{q}$ & & $0.31 \pm 0.09$ \\
\hline
\end{tabular}

Table 1: Global $\tau$ selection efficiency and non- $\tau$ backgrounds in the $\tau$ event selection at $Z^{0}$ peak energy. The values are obtained from Monte Carlo corrected with data. The errors include both statistical and systematic uncertainties.

\begin{tabular}{|c|r|r|r|}
\hline Id. $\downarrow \quad$ True $\rightarrow$ & \multicolumn{1}{|c|}{$e$} & \multicolumn{1}{c|}{$\mu$} & \multicolumn{1}{c|}{$h$} \\
\hline \hline$e$ & $99.49 \pm 0.10$ & $\leq 0.01$ & $0.79 \pm 0.06$ \\
\hline$\mu$ & $\leq 0.01$ & $99.32 \pm 0.10$ & $0.90 \pm 0.06$ \\
\hline$h$ & $0.51 \pm 0.10$ & $0.68 \pm 0.10$ & $98.31 \pm 0.08$ \\
\hline
\end{tabular}

Table 2: Particle identification efficiencies and misidentification probabilities (in percent) as measured from data in one-prong $\tau$ decays for particles with momentum above $2 \mathrm{GeV} / \mathrm{c}$ and avoiding the cracks between ECAL modules. 
The performance of this identification has been studied in detail using Bhabha, $\mu$-pair and $\gamma \gamma$ induced lepton pair samples over the full angular and momentum range above $2 \mathrm{GeV} / \mathrm{c}$ [4]. In addition, two complementary data samples have been used to understand and test the hadron misidentification probability. The first sample is obtained using the $d E / d x$ measurement to veto electron candidates (muons are rejected by HCAL and the muon chamber estimators) and the second one is tagged by the presence of at least one reconstructed $\pi^{0}$. Both samples indicate a higher probability for the misidentification of hadrons as electrons with respect to the Monte Carlo expectation, with some momentum dependence. After the convolution of this hadron misidentification probability with the hadron momentum distribution from $\tau$ decays, the value $(0.59 \pm 0.02) \%$ is obtained for the Monte Carlo while $(0.79 \pm 0.06) \%$ is measured from data. The identification efficiencies and the misidentification probabilities are corrected using these measurements. Table 2 shows the efficiency matrix for one-prong $\tau$ decays used for this analysis, i.e., for particles with a momentum larger than $2 \mathrm{GeV} / \mathrm{c}$ and not in a crack region. The particle identification is extended below the $2 \mathrm{GeV} / \mathrm{c}$ momentum region only for electrons because of the good $d E / d x$ separation in this region.

Figure 2 shows the distribution of the measured hadron identification estimator $P_{h}$ [4] with the Monte Carlo expectation, both for the identified hadron samples. According to the likelihood method introduced in reference [4], charged hadrons are identified as such if $P_{h}$ exceeds the value 0.5. Charged particles identified as electrons or muons ( $P_{h}$ less than 0.5$)$ are assigned to be hadrons if a $\pi^{0}$ is reconstructed in the same hemisphere. Also charged particles below $2 \mathrm{GeV} / \mathrm{c}$ or traversing a crack between ECAL modules are classified as hadrons if a reconstructed $\pi^{0}$ is present. The hadron estimator for the tagged hadrons above $2 \mathrm{GeV} / \mathrm{c}$ is also shown in Figure 2.

The hadron and electron identification is also tested separately in the three-prong $\tau$ hemispheres using conversions reconstructed topologically. In this case, the electron efficiency is measured to be $(97.8 \pm 0.3) \%$ from data and $(98.8 \pm 0.1) \%$ in the simulation. The effect of this discrepancy is taken into account in the systematic errors in Section 9.2. The hadron efficiency is found to be correctly described by the simulation. The kaon/pion separation using $d E / d x$ is used for the analysis of $\tau$ decays into kaons, as described in reference [5].

\section{Photons}

In this section the photon identification is described. First the identification of converted photons is explained. Secondly, the reconstruction procedure for photons developing a shower in the electromagnetic calorimeter is given. The high collimation of $\tau$ decays quite often makes photon reconstruction difficult, since these photons are close to one another or close to the showers generated by charged hadrons. Of particular relevance is the rejection of fake photons which may occur because of hadronic interactions, electromagnetic shower fluctuations, or the overlapping of several showers. In this section, a procedure to overcome this problem is 

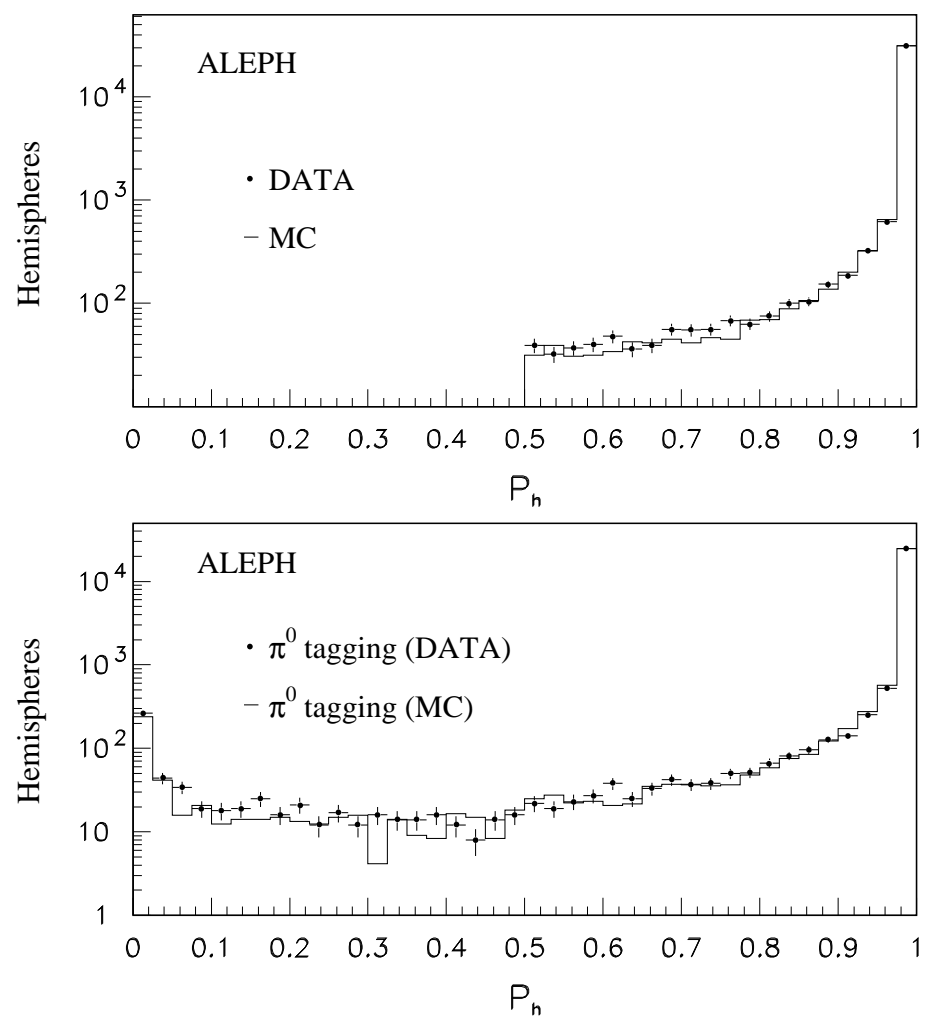

Figure 2: Distribution of the identification estimator for charged hadrons with a momentum larger than $2 \mathrm{GeV} / \mathrm{c}$ in one-prong hemispheres. Points with error bars show the observed distribution and the solid histogram corresponds to the simulation. In the upper plot only particles identified by the likelihood method are shown. The lower plot corresponds to the particles accompanied by a reconstructed $\pi^{0}$. The part of the distribution below 0.5 corresponds to hadrons which would be misidentified in the absence of a $\pi^{0}$.

presented.

\subsection{Converted photons}

In order to identify photons which convert inside the tracking volume all oppositely charged track pairs of a given hemisphere in which at least one track is identified as an electron are considered. These candidates are required to have an invariant mass smaller than $30 \mathrm{MeV} / \mathrm{c}^{2}$ and the minimal distance between the two helices in the $\mathrm{x}-\mathrm{y}$ plane must be smaller than $0.5 \mathrm{~cm}$. Finally, all remaining unpaired charged tracks identified as electrons are kept as single track photon conversions. These include Compton scatters or asymmetric conversions where the other track was either lost or poorly reconstructed. 

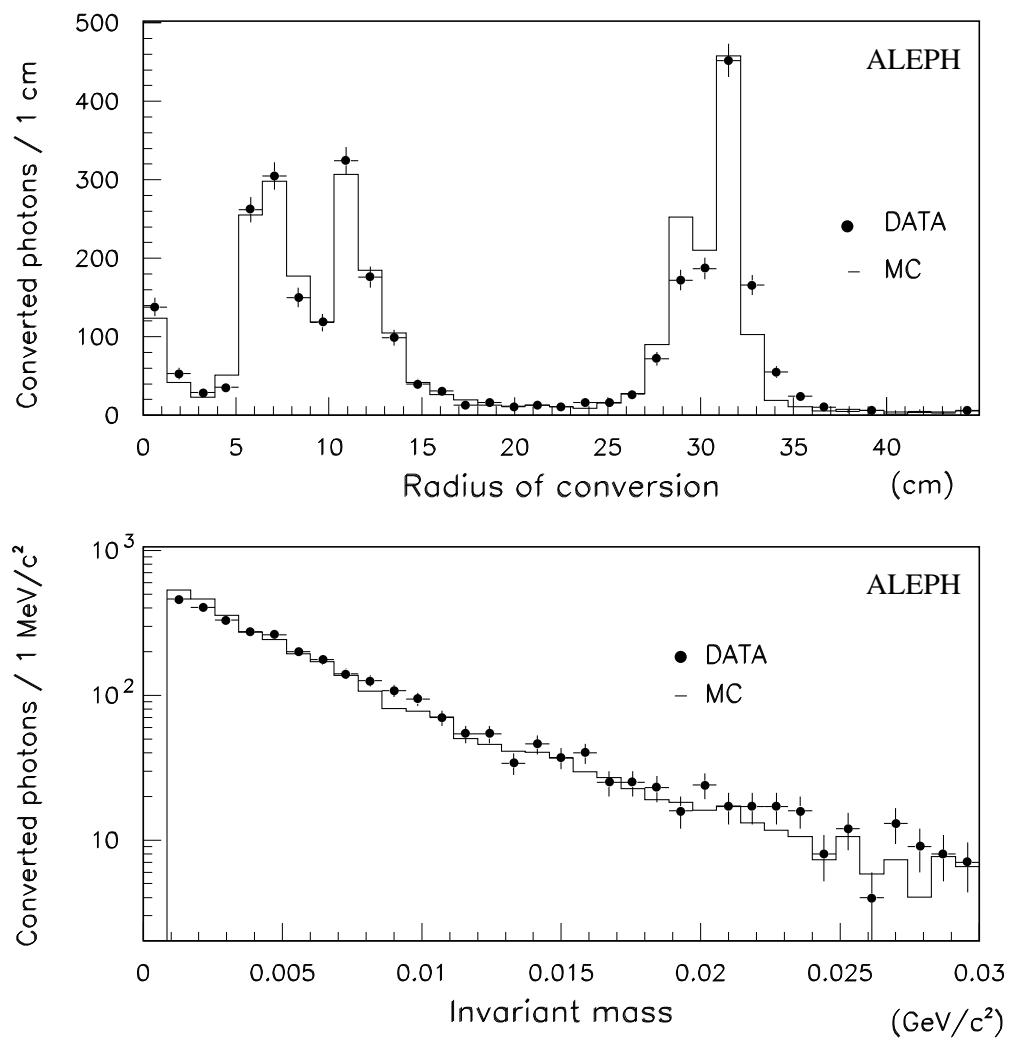

Figure 3: The upper plot shows the radial distance to the beam axis for the converted photons for the simulation (solid histogram) and for the observed converted photons (points with error bars). This material description corresponds to the beam pipe $(5.4 \mathrm{~cm}), \operatorname{VDET}(6-11 \mathrm{~cm})$, inner and outer ITC walls $(13,29 \mathrm{~cm})$, and the inner TPC wall $(31 \mathrm{~cm})$. Dalitz decays are also observed at the origin. The lower plot shows the invariant mass distribution for the observed and simulated converted photons.

The radial distribution of the materialization point for the observed converted photons shows in Figure 3 that the amount of material in the detector is properly modelled, with the exception of the outer ITC and inner TPC walls. The invariant mass distribution for the observed conversions is also shown. The fraction of converted photons with respect to genuine photons is measured in data to be $(9.8 \pm 0.2) \%$, whilst in the Monte Carlo it is $(9.4 \pm 0.1) \%$. This possible discrepancy is analyzed further in Section 9.2. A detailed comparison of data and Monte Carlo is made for the two classes of conversions (electron-electron and electron with a non-identified particle) to test the particle identification entering the definition of converted photons. A good agreement is observed. The fraction of single track conversions is also well reproduced. No discrepancy with the simulation is found in spite of the small overall excess of converted photons from data.

Figure 4 shows the energy distribution for the single track and two-track converted photons 

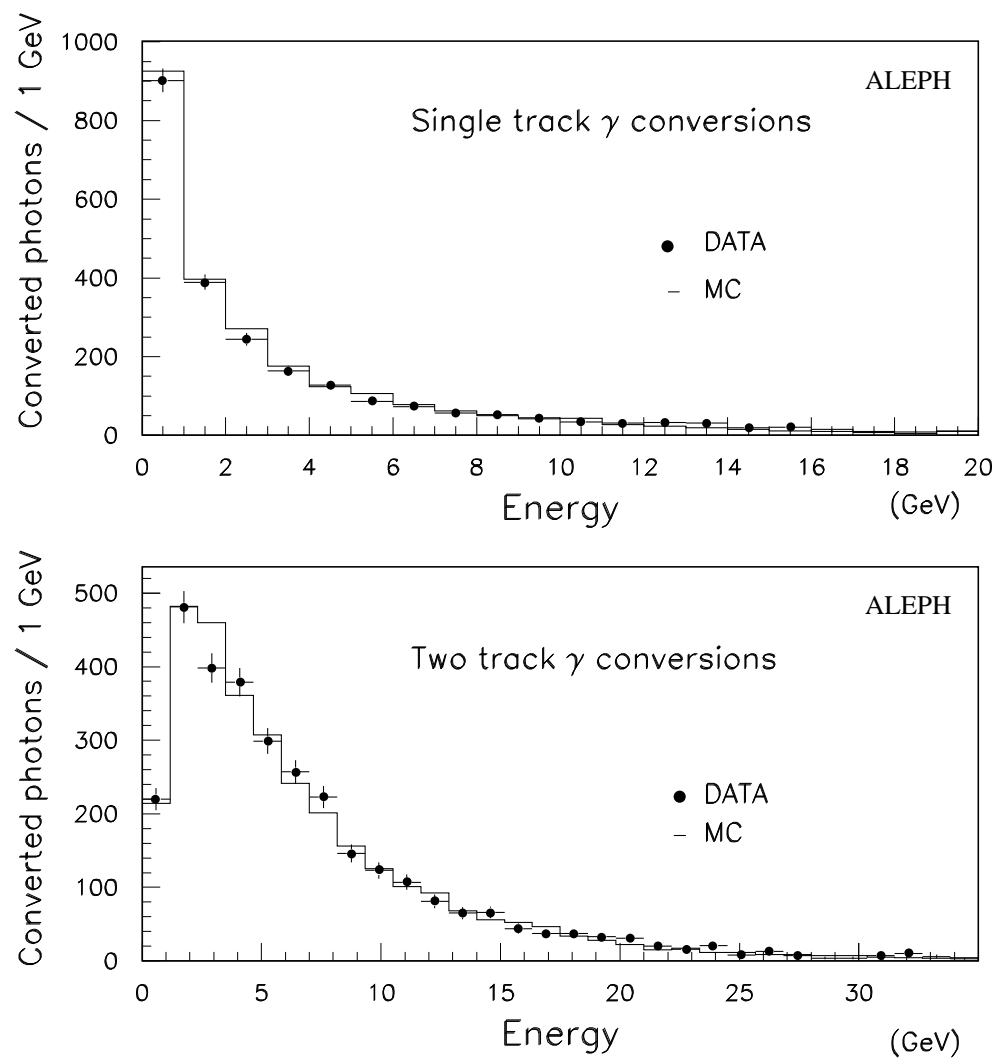

Figure 4: Energy spectra for the single track (upper distribution) and two-track (lower distribution) converted photons (points with error bars). The solid line corresponds to the simulation.

in good agreement with the Monte Carlo expectation.

\subsection{Photon reconstruction}

The clustering algorithm for the photon reconstruction [8] starts with a search for local maxima among the towers in the three ECAL stacks. The segments of a projective tower which share a face in common with the local maximum are linked together into a cluster. At the end of the procedure, every segment of a tower is clustered with its neighbour of maximal energy. Then, a cluster is accepted as a photon candidate if its energy exceeds $350 \mathrm{MeV}$ and if its barycentre is at least $2 \mathrm{~cm}$ away from the closest charged track extrapolation. Some events appear below this value (Figure 5) because the distance is recalculated after corrections for the finite size of the pads. The energy of the photon is calculated from the energy of the four central towers only when the energy distribution of the cluster is consistent with the expectation of a single photon, otherwise the sum of the tower energies is taken. The direction of the photon is determined from the barycentre of energy deposition. 
After the clustering procedure, the number of fake photons reaches $20 \%$ over the whole photon sample according to the Monte Carlo simulation, depending on the hadron final state. The fractions of fake photons originating from hadronic interactions and electromagnetic showers are approximately $60 \%$ and $40 \%$, respectively.

\subsection{Likelihood method}

In order to distinguish fake photons from photons originating from $\pi^{0}$ decays or other physical sources several estimators are constructed and a likelihood method is used. In fact, the likelihood method is found to be less sensitive to systematic effects on the reference distributions with respect to the procedure that consists of applying cuts on the same discriminating distributions. For every photon the following estimator is defined:

$$
P_{\gamma}=\frac{P^{\text {genuine }}}{P^{\text {genuine }}+P^{\text {fake }}},
$$

where $P^{i}$ is the estimator under the photon hypothesis of type $i$, which is given by

$$
P^{i}=\prod_{j} \mathcal{P}_{j}^{i}\left(x_{j}\right)
$$

and $\mathcal{P}_{j}^{i}$ is the probability density for the photon hypothesis of type $i$ associated to the discriminating variable $x_{j}$.

Several discriminating variables are used to distinguish between genuine and fake photons:

- fractions of energy in the first and second stacks of ECAL,

- fraction of energy outside the four central ECAL towers,

- transverse size of the photon shower,

- angular distance $\left(d_{\gamma \gamma}\right)$ to the nearest photon,

- distance between the barycentre of the photon and the closest charged track. A sign is computed depending on the position of the photon shower with respect to the track bending in the $r-\phi$ projection.

- energy of the photon.

Reference distributions of these discriminating variables have been established from the Monte Carlo simulations for genuine and fake photons, depending on the number of photons and charged tracks in the hemisphere. For this purpose, in the simulation sample, the reference distributions for genuine photons are set up with photons whose physical origin 

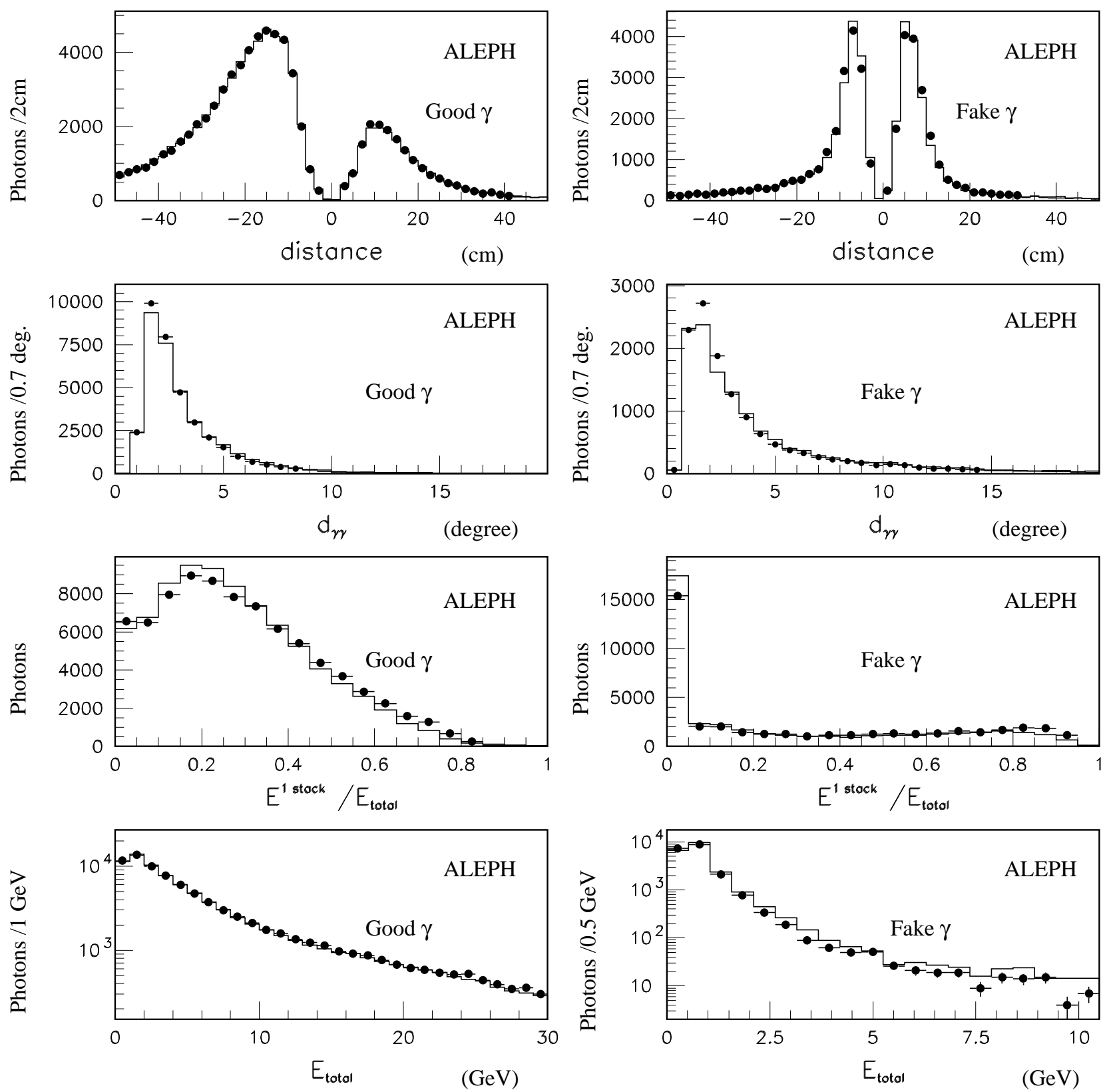

Figure 5: Data and Monte Carlo distributions of some of the discriminating variables used for the photon identification. The upper figures (left and right) show the distributions of the distance between a photon and the closest charged track (labeled "distance") and the minimal angular distance between two photons (labeled " $d_{\gamma \gamma}$ ") for good and fake photons respectively. The distribution for the fraction of energy deposited in the first stack for good and fake photons is given in the middle figures; whilst the energy spectra are shown in the lowest figures. Note the different scales in the last two plots. Points with error bars show the distributions for observed photons and the solid histograms the simulated ones. In each plot the distributions for data and Monte Carlo are normalized to the same number of photons. 

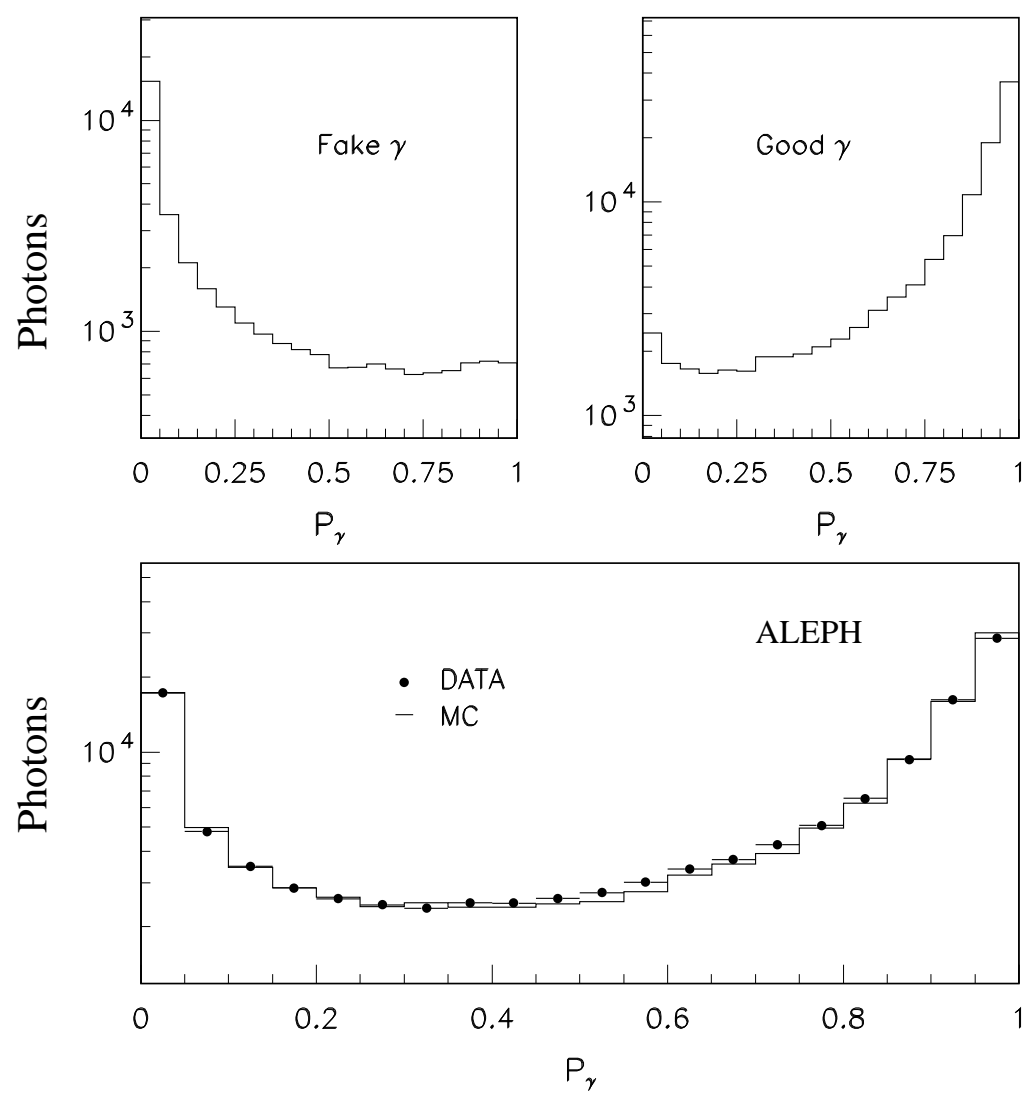

Figure 6: In the upper plots, the $P_{\gamma}$ distribution for good and fake photons from the simulation. In the lower distribution the points with error bars show the $P_{\gamma}$ distribution for all the observed photons and the solid histogram shows this distribution from the simulation.

(radiation, $\pi^{0}$ decay, etc.) is determined by the information at the generator level. The remaining reconstructed photons with no identified source are declared fake and used for the corresponding reference distributions.

All these reference distributions have been confronted with data. For this purpose, a criterion based on the value of the $P_{\gamma}$ estimator together with the information whether the photon belongs or not to a $\pi^{0}$ is used for data and Monte Carlo. Figure 5 shows the distributions for genuine and fake photons for some of the discriminating variables, namely the distance between the photon and the nearest charged track, the minimal distance between two photons, the fraction of energy in the first stack and their energy spectra. Although the Monte Carlo is able to reproduce satisfactorily the distributions for genuine photons, Figure 5 shows that some discrepancies appear in the distributions for fake photons. An iterative procedure has been used to derive from data the corrections to be applied to the reference distributions.

Figure 6 shows the distributions for the $P_{\gamma}$ estimator which distinguishes genuine and fake 
photons. In the computation of $P_{\gamma}$, at this stage, the energy of the photon is not used in order to avoid a bias for the low energy photons from $\pi^{0}$ decays. From a linear fit of the observed $P_{\gamma}$ distribution to the distributions for genuine and fake photons shown in the upper plots of Figure 6, it is found that the Monte Carlo simulation underestimates the number of fake photons by $(16 \pm 2) \%$. The lower plot in Figure 6 shows the $P_{\gamma}$ distribution for data and Monte Carlo after the fraction of fake photons is increased by this amount. The shape of the distributions are in agreement. The systematic effect in the branching ratio analysis induced by the discrepancy on the fraction of fake photons is treated in Section 9. It should be noticed that no cut is applied at the initial level of the likelihood procedure to reject photons with low values of the $P_{\gamma}$ estimator.

\section{$6 \quad \pi^{0}$ reconstruction}

The goal of the $\pi^{0}$ reconstruction procedure is to reach the highest possible efficiency. Three types of $\pi^{0}$ s are considered here:

- first, photons are paired to reconstruct $\pi^{0}$ s (Section 6.1, "resolved $\pi^{0}$ 's"),

- high energy $\pi^{0}$ 's often lead to one single cluster in the electromagnetic calorimeter, and are searched for separately (Section 6.2, "unresolved $\pi^{0}$ s"),

- finally, because of the loss of one of the photons some of the $\pi^{0}$ s appear as a single photon (Section 6.3, "residual single photons").

In totality, these procedures lead to an overall efficiency of $\sim 84 \%$ for finding $\pi^{0}$ 's.

\subsection{Resolved $\pi^{0}$ 's}

The first step of $\pi^{0}$ reconstruction is the pairing of all photon candidates within one hemisphere, considering all possible combinations. Only photons inside a cone of $45^{\circ}$ around the thrust axis are considered for the pairing. A $\pi^{0}$ identification estimator $D_{i, j}^{\pi^{0}}$ for two photons $i$ and $j$ is defined in the following way:

$$
D_{i, j}^{\pi^{0}}=P_{\gamma_{i}} \cdot P_{\gamma_{j}} \cdot P_{\pi^{0}}
$$

where $P_{\gamma_{i}}$ is the estimator for photon $i$ to be genuine according to equation (1) and $P_{\pi^{0}}$ is the probability coming from a kinematic $\pi^{0}$-mass constrained fit.

An observed energy dependence of the $\pi^{0}$ mass is derived from fits of $\gamma \gamma$ invariant mass distributions in different energy bins. Above $10 \mathrm{GeV}$ the showers from the two photons tend 


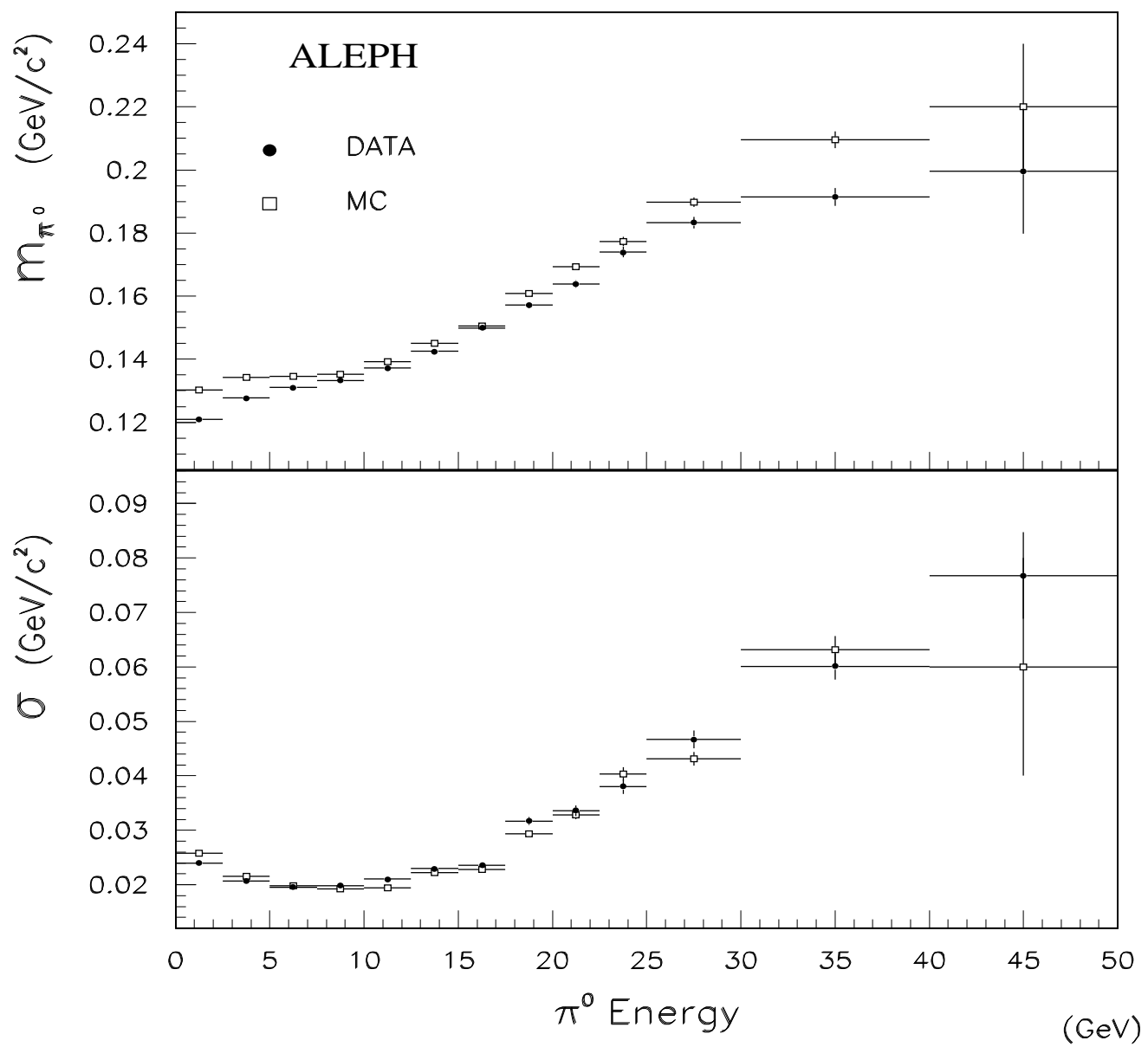

Figure 7: The upper plot shows the observed mean $\pi^{0}$ mass as a function of the $\pi^{0}$ energy for the data (points with error bars) and for the simulation (open squares). The lower plot shows the resolution of the $\pi^{0}$ mass as a function of the $\pi^{0}$ energy.

to overlap in the calorimeter. Thus, the sample of resolved $\pi^{0}$ 's at high energy is biased towards larger masses due to a systematic overestimate of the opening angle. This explains the trend observed in the upper plot of Figure 7 for both data and Monte Carlo. This effective $\pi^{0}$ mass dependence is taken into account for the constrained fit used to obtain $P_{\pi^{0}}$.

In spite of the general dependence in agreement with the simulation, small but significant differences exist which are taken into account. On one hand, at high $\pi^{0}$ energy the opening angle between the two photons is slightly overestimated in the simulation. On the other hand, the lower $\pi^{0}$ mass observed in the data at low energy is due in part to the excess of fake photons in data producing on average lower masses, and in part to low energy photon calibration. Thus, the effective $\pi^{0}$ mass dependence is taken separately for data and Monte Carlo.

A detailed study has also been performed for the energy resolution of the photons 

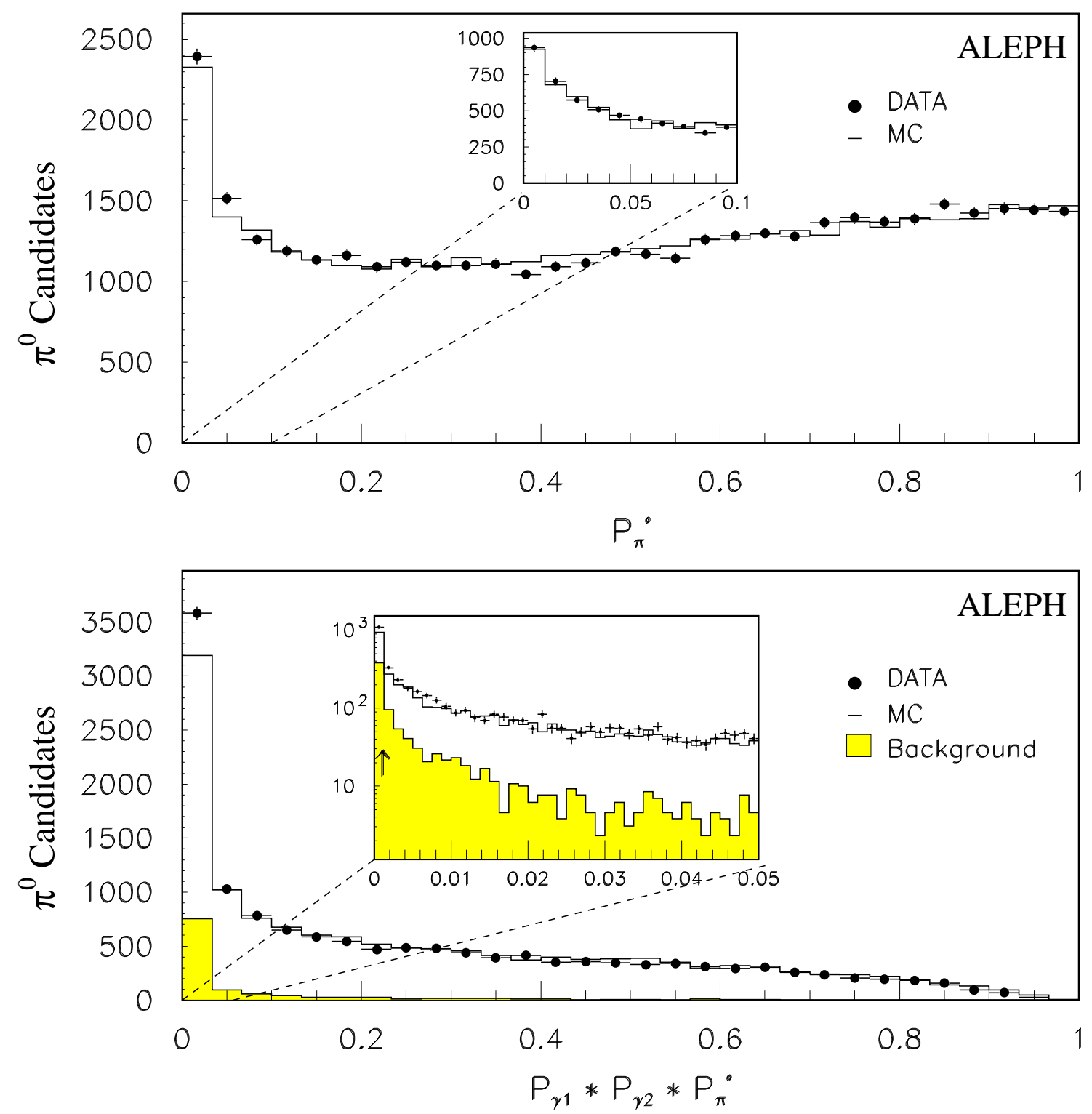

Figure 8: The upper figure shows the comparison of the $P_{\pi^{0}}$ probability distribution for the resolved $\pi^{0}$ candidates in data and Monte Carlo. In the lower figure, the distributions of $D^{\pi^{0}}$ for the data $\pi^{0}$ 's (points with error bars) and for the simulation (solid histogram) are given. The shaded histogram shows the expected background coming from either the wrong pairing of good photons or the pairing with fake photons. From all the possible $\gamma_{i} \gamma_{j}$ combinations, only the $\pi^{0}$ candidates retained in the final decay configuration are plotted. 


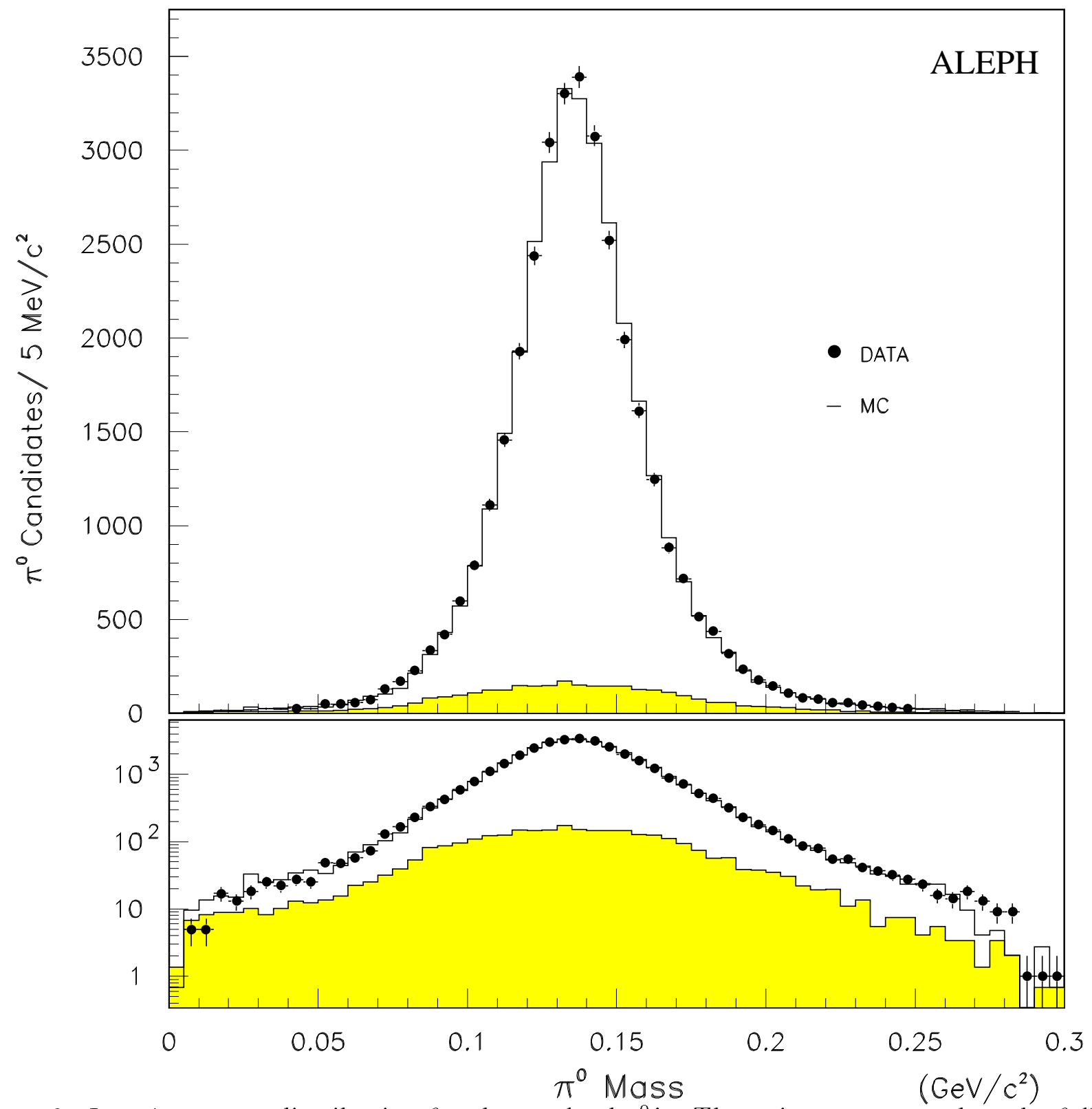

Figure 9: Invariant mass distribution for the resolved $\pi^{0}$ 's. The points correspond to the full data sample and the solid histogram shows the simulated $\pi^{0}$ 's. The shaded histogram shows the expected background. 
depending on the kinematics. The lower plot of Figure 7 shows the resolution for the $\pi^{0}$ mass as a function of the $\pi^{0}$ energy. In the case where one of the paired photons is a converted photon other parameterizations for the $\pi^{0}$ mass and resolution are derived from data to compute the $P_{\pi^{0}}$ probability, since these photons have a different resolution and are potentially subject to different systematic effects.

A pair of photons is considered to be a $\pi^{0}$ candidate if the $D_{i, j}^{\pi^{0}}$ value is greater than 0.0009. For an average value of $P_{\gamma_{i}} \cdot P_{\gamma_{j}}$ for a pair of genuine photons, this corresponds to a $P_{\pi^{0}}$ threshold probability for an invariant mass three standard deviations away from the expected value. Figure 8 shows the comparison of the $P_{\pi^{0}}$ probability distribution for Monte Carlo and data, in good agreement. The lower plot of this figure shows the $D_{i, j}^{\pi^{0}}$ distributions with the expected background from incorrect pairings.

In addition, a criterion must be established for choosing among all the accepted $i-j$ pairs in a multiphoton environment. The overall configuration for the maximum number of $\pi^{0} \mathrm{~s}$ allowed given the photon multiplicity is chosen to maximize the product of all $D_{i, j}^{\pi^{0}}$. Then, only the photon pairs whose $D_{i, j}^{\pi^{0}}$ values satisfy the criterion previously mentioned are taken definitively as $\pi^{0}$ 's. The comparison of the invariant mass distribution for the resolved $\pi^{0}$ 's, once the $\pi^{0}$ mass shift between the simulation and data is corrected, is plotted in Figure 9. The shapes of the distributions are in excellent agreement.

Once the resolved $\pi^{0}$ 's are identified, a second kinematic constrained fit is performed to the nominal $\pi^{0}$ mass, which allows a better determination of the $\pi^{0}$ energy as shown in Figure 10. This second fit allows to compensate the low energy threshold effects. The remaining photons that were not paired are treated as discussed next.

\subsection{Unresolved $\pi^{0}$ s}

As the $\pi^{0}$ energy increases it becomes more difficult to resolve the two photons and the clustering algorithm may yield a single cluster. The two-dimensional energy distribution in the plane transverse to the shower direction is examined and energy-weighted moments are computed. Assuming only two photons are present, the second moment provides a measure of the $\gamma \gamma$ invariant mass. Figure 11 shows this invariant mass distribution for Monte Carlo simulation and data, in excellent agreement. For photon energies lower than $8 \mathrm{GeV}$ the two photons are expected to always be resolved by the clustering algorithm and the mass distribution only reflects fluctuations in single showers. As the photon energy increases this technique reveals a wide peak at the $\pi^{0}$ mass. All single clusters not entering the $\pi^{0}$ reconstruction in Section 6.1 but having an invariant mass larger than $100 \mathrm{MeV} / \mathrm{c}^{2}$ according to this method are kept as $\pi^{0}$ candidates. It must be stressed that this procedure does not allow a clear separation between high energy radiative photons and high energy $\pi^{0}$ 's when the $\gamma \gamma$ separation is comparable to the transverse shower size. However, in $\tau$ events such energetic photons originate predominantly from $\pi^{0}$ decays. 


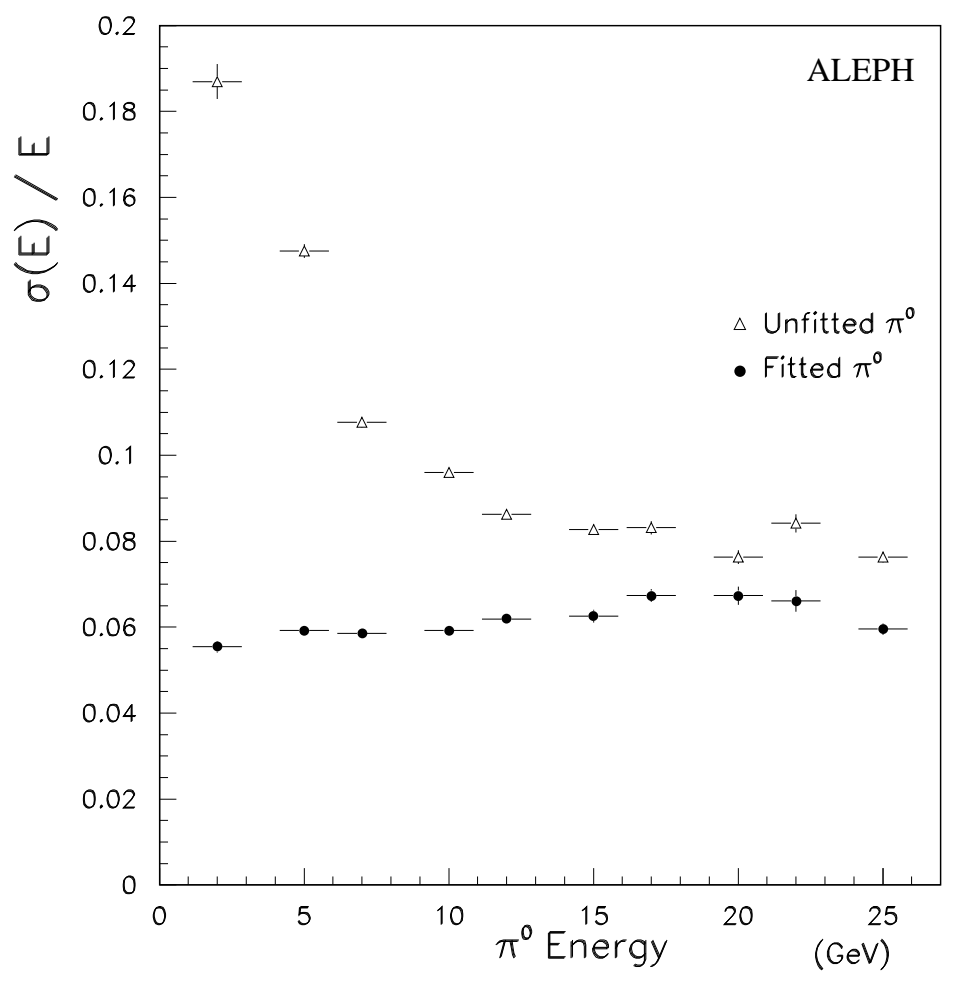

Figure 10: Energy resolution of the exclusive $\pi^{0}$ 's as a function of energy before and after the kinematic fit according to the simulation.

\subsection{Residual single photons}

After the pairing of photons and the cluster moment analysis, all the remaining photons inside a cone of $30^{\circ}$ around the thrust axis are called residual single photons. They come from several sources:

- bremsstrahlung photons from radiation along the final charged particle in $\tau$ decay (including the detector material for electrons),

- initial and final state radiation,

- genuine photons from $\pi^{0}$ decays where the partner photon is lost because of threshold, cracks or overlap with another electromagnetic or hadronic shower,

- genuine photons from $\omega \rightarrow \pi^{0} \gamma$ and $\eta \rightarrow \gamma \gamma$ decays,

- fake photons.

The fraction of fake photons in the residual single photon sample is observed to be about $50 \%$. 


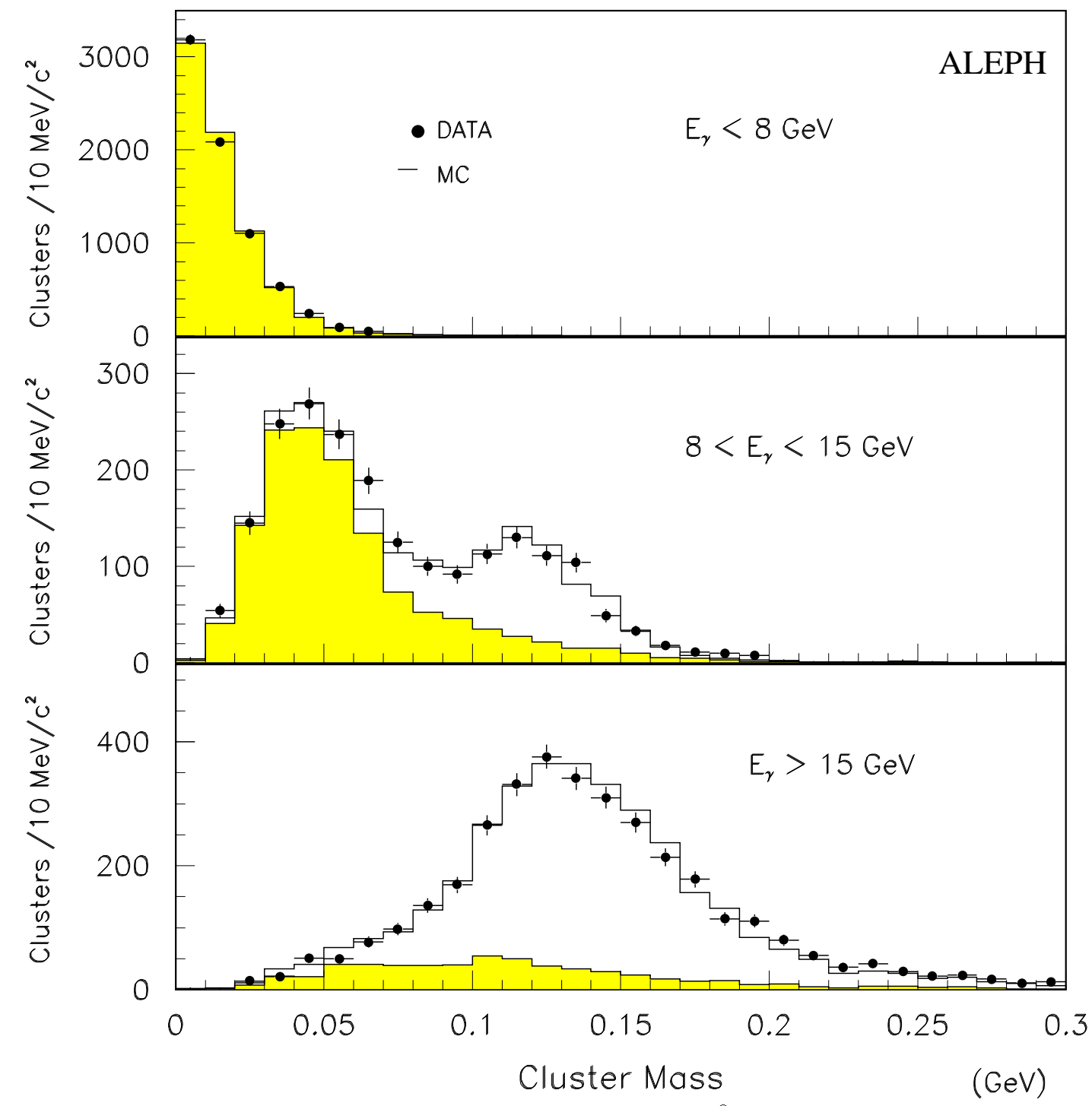

Figure 11: Invariant mass distributions for the unresolved $\pi^{0}$ 's in three different photon energy ranges. Points with error bars come from data and the solid histogram shows the distribution from the simulation. The shaded histogram corresponds to single photons (radiative photons or $\pi^{0}$ decays with a lost photon). 

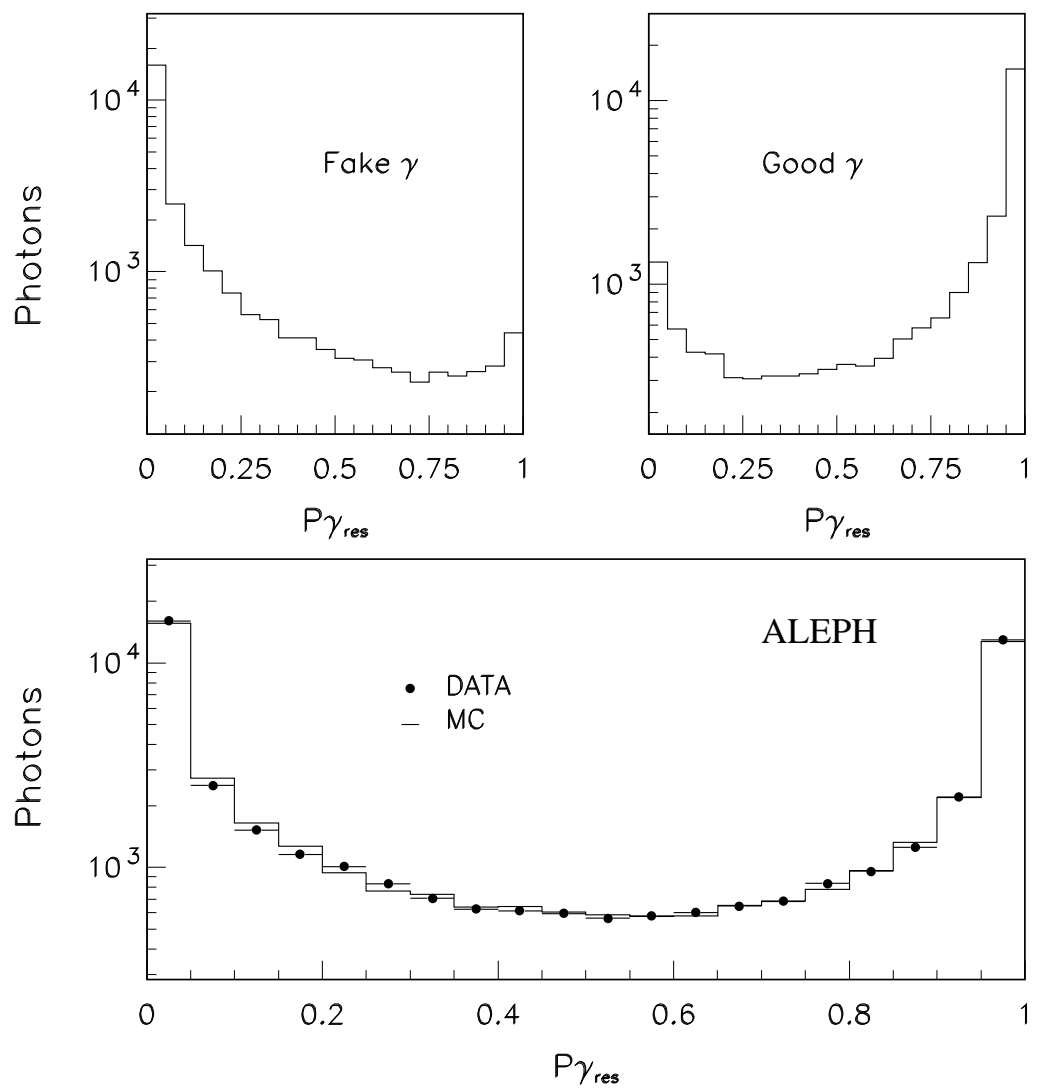

Figure 12: $P_{\gamma_{R e s}}$ distribution for good and fake photons from the simulation. In the lower distribution, the points with error bars show the $P_{\gamma_{\text {Res }}}$ distribution for all the observed photons and the solid histogram is from the simulation.

To proceed with the removal of these fake photons, another estimator $P_{\gamma_{\text {Res }}}$ is computed along the lines described in Section 5, but this time the energy of the photon is used and the reference distributions are set up depending on the numbers of charged hadrons, reconstructed $\pi^{0}$ 's and residual photons. The behaviour of this estimator is shown in Figure 12 for the fake and genuine photons. To obtain a good description of the full $P_{\gamma_{R e s}}$ distribution the fraction of fake photons in the Monte Carlo must be increased by $(17 \pm 2) \%$, consistent with the previous determination. After this normalization the shapes of the Monte Carlo and data distributions agree well. Photons with a value of $P_{\gamma_{R e s}}$ smaller than 0.5 are declared fake and are therefore rejected. In this procedure $90 \%$ of the fake photons are rejected, whilst $18 \%$ of genuine photons are lost. Systematic checks are carried out to assess the validity of the probability densities used in the calculation of estimators and some correction functions are estimated from data to account for the observed discrepancies between Monte Carlo and data following the procedure described in Section 5.3.

To distinguish among the different physical sources feeding the sample of genuine residual single photons, new estimators $P_{B r e m}, P_{R a d}$ and $P_{\pi^{0} \rightarrow \gamma}$ are calculated to select photons from 

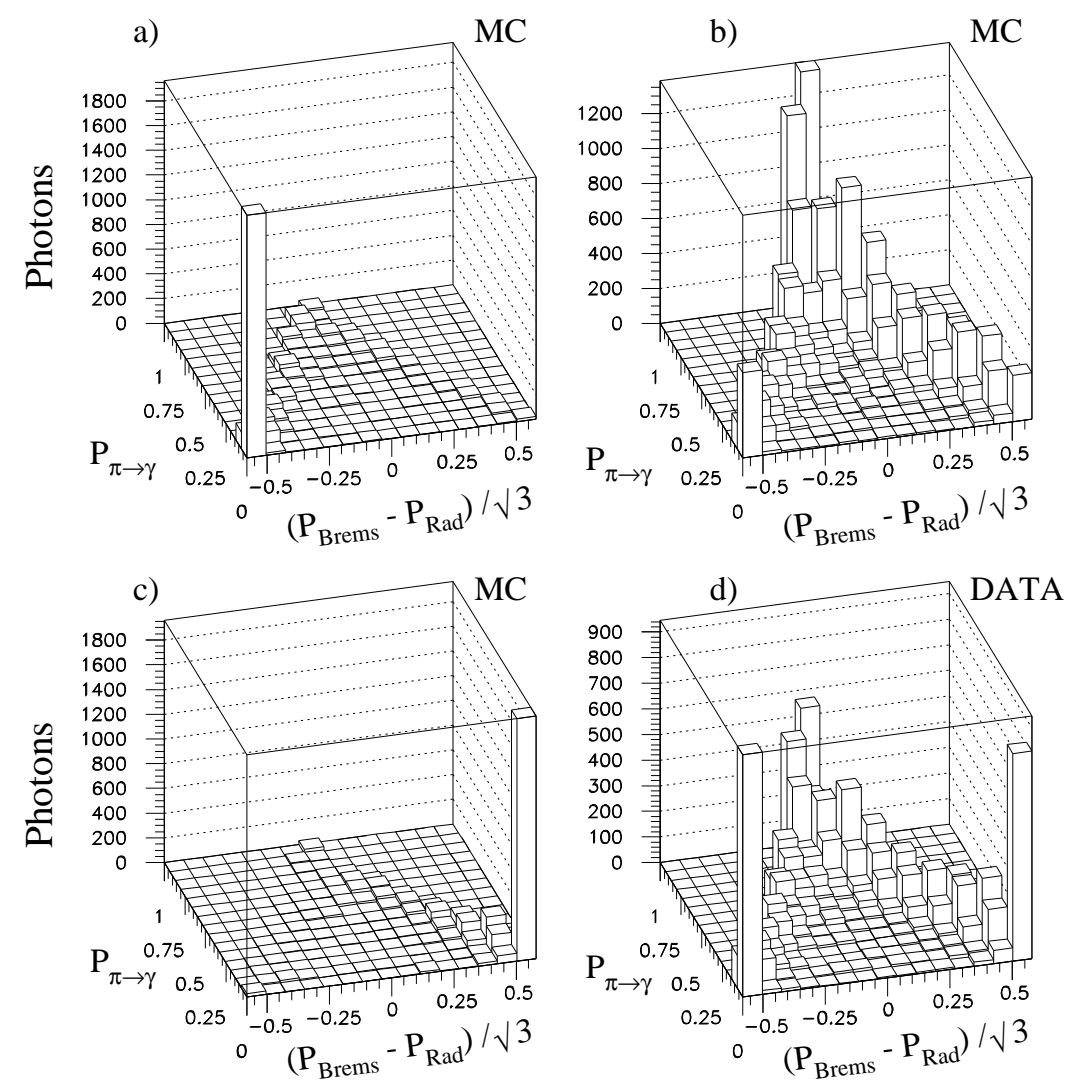

Figure 13: Figures a), b) and c) show triangular plots for the probabilities in the simulation for a single photon coming from an initial or final state radiation process, or from a $\pi^{0}$ decay, or from bremsstrahlung, respectively, to be identified as one of these three sources. Figure d) gives the same plot for the selected single photons in the data.

bremsstrahlung processes, from radiative processes and from $\pi^{0}$ decays, respectively. To compute these estimators, the angle between the photon and the most energetic charged track is used, in addition to the discriminating variables introduced in Section 5.3. The behaviour of those estimators is shown in Figure 13 for Monte Carlo and data. Residual single photons with a $P_{\pi^{0} \rightarrow \gamma}$ value larger than 0.07 are declared single photons coming from $\pi^{0}$ decay. The remaining single photons with $P_{\pi^{0} \rightarrow \gamma}$ smaller than 0.07 are classified as bremsstrahlung photons or radiative photons according to values of $P_{B r e m}$ and $P_{R a d}$. Possible systematics uncertainties arising from the reference distributions have been studied by comparing Monte Carlo and data estimators and distributions for the different configurations. The overall agreement is found to be satisfactory. The number of radiative photons found in the data sample is $1652 \pm 41$ which compares well with the $1659 \pm 33$ predicted by the Monte Carlo. The corresponding numbers for the bremsstrahlung photons are $1640 \pm 40$ and $1672 \pm 33$, mostly concentrated in the electron channel. Radiative and bremsstrahlung photons are not used in the $\tau$ decay classification discussed in the next section. 


\subsection{Summary on $\pi^{0}$ reconstruction}

The fractions of resolved $\pi^{0}$ 's, unresolved $\pi^{0}$ 's and single photons coming from a $\pi^{0}$ decay are shown in Figure 14 as a function of the $\pi^{0}$ energy. For the resolved $\pi^{0}$ 's, the contribution of the $\pi^{0}$ decays where at least one of the photons is converted into a $e^{+} e^{-}$pair is observed to be around $14 \%$ above $10 \mathrm{GeV}$ in good agreement with the simulation. The fraction of resolved $\pi^{0}$ 's without converted photons remains at a relatively high level above $25 \mathrm{GeV}$ considering the granularity of the electromagnetic calorimeter. This is caused by the fact that the corresponding showers quite often have large fluctuations yielding two separate photon candidates in the clustering algorithm. This effect is not well reproduced by the Monte Carlo, where this shower splitting occurs less frequently. The excess of resolved $\pi^{0}$ 's at high energy corresponds to a deficit in the unresolved $\pi^{0}$ fraction, so that the sum of the resolved and unresolved $\pi^{0}$ contributions is well described by the simulation. Apart from the high $\pi^{0}$ energy region, the fractions of resolved and unresolved $\pi^{0}$ 's are reasonably well simulated. Also, a small excess of resolved $\pi^{0}$ 's is observed in data for $\pi^{0}$ energies smaller than $4 \mathrm{GeV}$ due to the aforementioned excess of fake photons.

In fact, Figure 14 illustrates the $\pi^{0}$ and photon treatment in the hadronic $\tau$ decay classification and the complementarity of different estimators. Resolved $\pi^{0}$ 's are reconstructed over a wide range of energy, with maximal efficiency between 5 and $15 \mathrm{GeV}$, while the cluster moment analysis is essential to retain a good $\pi^{0}$ efficiency above $20 \mathrm{GeV}$. On the other hand, at low $\pi^{0}$ energy, where often one of the photons is lost, the estimators described in the previous section recover a substantial fraction of the sample. Figure 15 shows the $\pi^{0}$ energy spectrum including the contributions from all three $\pi^{0}$ types. The agreement between data and Monte Carlo is satisfactory as the ratio indicates.

Including the detector acceptance, this treatment yields an overall " $\pi^{0}$ " efficiency of $83.7 \%$ with respect to all produced $\pi^{0}$ s $\mathrm{s}$ in the selected $\tau$ pair events with a background fraction of $8.7 \%$. This background is estimated with the Monte Carlo. Any " $\pi^{0}$ " candidate with either an energy $3 \sigma$ away from true value or differing from the true direction by more than $17 \mathrm{mrad}$ is classified as a fake $\pi^{0}$. For the three different $\pi^{0}$ types, the efficiencies are $55.1 \%, 10.5 \%$ and $18.1 \%$ for resolved $\pi^{0}$ s, unresolved $\pi^{0}$ 's and residual single photons, respectively. The backgrounds are $8.5 \%, 5.0 \%, 10.9 \%$, respectively. 


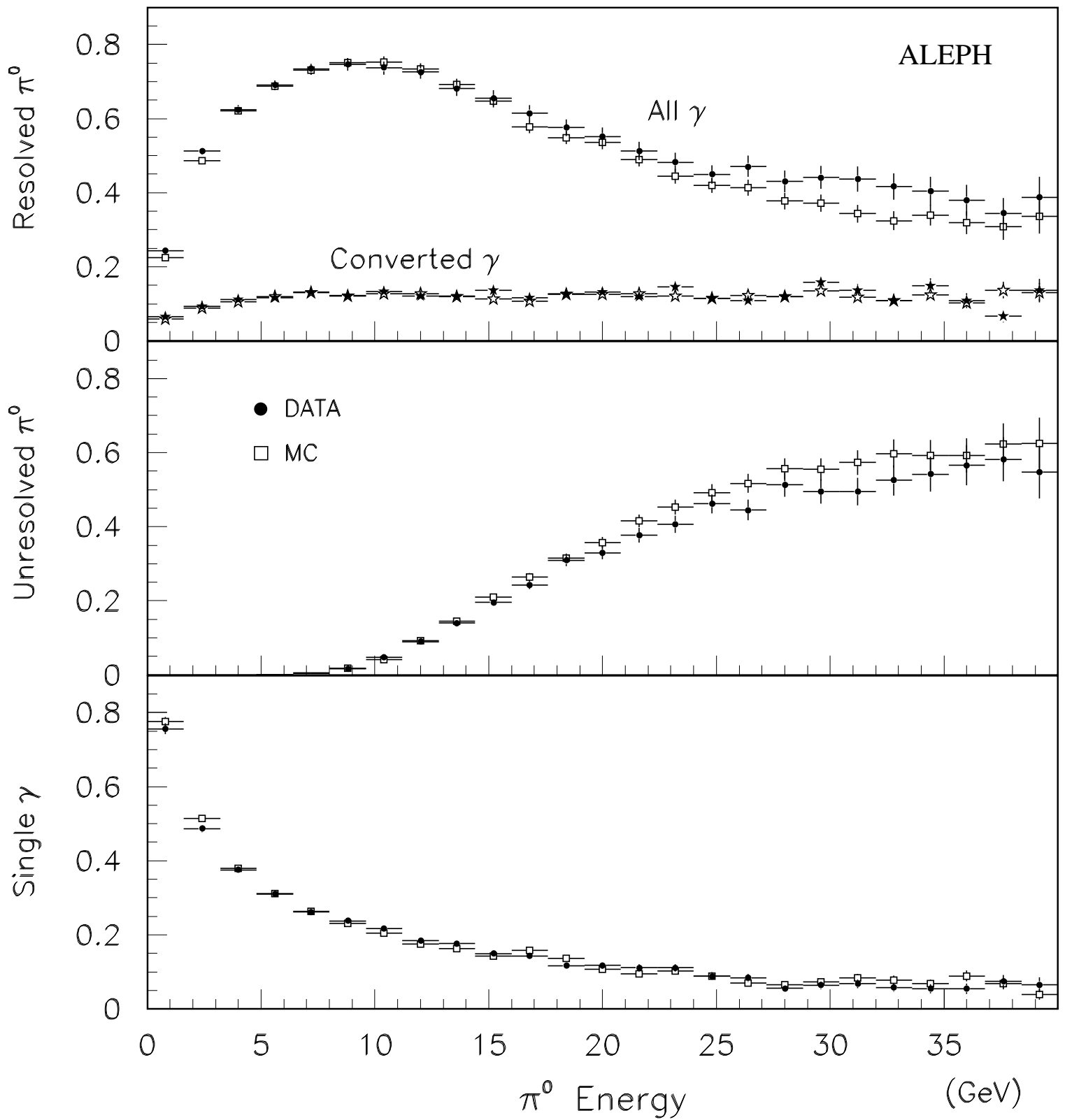

Figure 14: Fractions of resolved and unresolved $\pi^{0}$ and single photons as a function of the $\pi^{0}$ energy. The points represent the data, and the open squares the simulation. The proportion of $\pi^{0}$ 's containing at least one converted photon is plotted for the resolved $\pi^{0}$ 's; the full stars correspond to the data and the open ones to the simulation. 

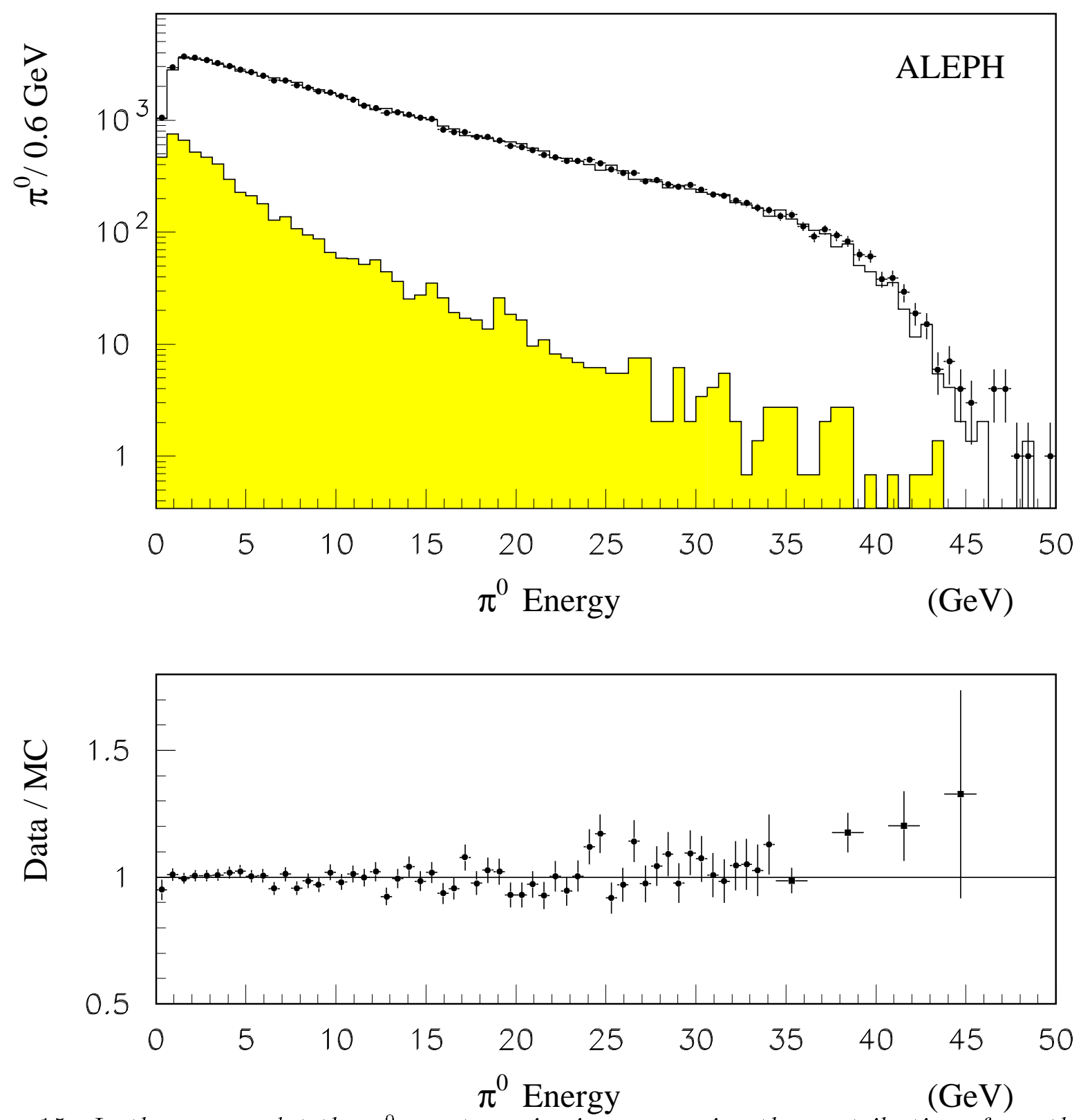

Figure 15: In the upper plot the $\pi^{0}$ spectrum is given summing the contributions from the three types of $\pi^{0}$ 's. For the resolved $\pi^{0}$ 's, the energy coming from the $\pi^{0}$ mass constraint is used. The points with error bar show the data and the solid histogram corresponds to the simulation. The shaded histogram refers to the $\pi^{0}$ 's containing fake photons or obtained from wrong pairings. The lower plot shows the ratio between data and Monte Carlo. 


\section{Tau decay classification}

Decays are classified unambiguously in one of the 13 classes which are schematically presented in Table 3 according to the number of charged tracks and their identification, and the number of reconstructed $\pi^{0}$ s. The leptonic $\tau$ decays are identified following the criteria described in reference [4]. Particle identification is not required in the three- and five-prong hemispheres, as discussed in Section 4. All selected $\tau$ decays are classified, except for single charged tracks going into an ECAL crack or with a momentum smaller than $2 \mathrm{GeV} / \mathrm{c}$ without a reconstructed $\pi^{0}$.

In Table 3, the right-most column shows how the different $\tau$ decays in the Monte Carlo contribute to the signal in each defined class at the reconstructed level. These events are processed through the full simulation chain and their feedthrough among different classes is calculated.

A poor modeling of the dynamics of some $\tau$ decays in the simulation can lead to systematic effects in the calculation of the relative efficiencies. Such uncertainties, when relevant, are discussed in Section 9.2. In fact, some progress has recently been made in several $\tau$ decays such as $\tau \rightarrow 3 \pi 2 \pi^{0} \nu_{\tau}$ [12], found to be dominated by the $\omega$ resonance, and the observation of the decay $\tau \rightarrow \pi \pi^{0} \eta \nu_{\tau}[13]$, both implemented in the simulation.

So far no attempt is made in this classification to explicitly take into account charged and neutral kaons. As the inclusive $\tau$ decays involving strange particles amount to $3.5 \%$, they can play a significant role in some particular channels, and their effect is included. A dedicated analysis was performed for the $\tau$ decays involving kaons in the final state $[5,6]$ and an update is given in Section 11. In the global analysis, a $K_{S}^{0} \rightarrow \pi^{+} \pi^{-}$decay counts as two hadrons at the generator level, since in general one charged pion is attached to the main vertex, and one-prong final states with $K_{S}^{0}$ 's are classified in the $\geq 2$ prong topology. A $K_{S}^{0} \rightarrow \pi^{0} \pi^{0}$ decay counts as two $\pi^{0}$ 's, whilst the $K_{L}^{0}$ 's is not taken into account in the classification of Table 3 . The non $-\pi^{0}$ photons from $\eta$ or $\omega$ decays are considered as $\pi^{0}$ 's. Because of the different kinematics the efficiencies for the modes with kaons are not identical to those with only pions. For this reason the efficiency for an identified class contains implicitly a weight depending on the branching ratio of the involved strange particles. In the global analysis, the Monte Carlo simulation uses branching ratio values in agreement with the ALEPH measurements of $\tau$ decays into kaons, which are summarized in Table 14. For the decays $\tau^{-} \rightarrow K^{-} \pi^{+} \pi^{-} \nu_{\tau}$ and $\tau^{-} \rightarrow K^{-} K^{+} \pi^{-} \nu_{\tau}$, which are not yet measured by ALEPH, the values $(0.39 \pm 0.12) \%$ and $(0.17 \pm 0.07) \%$ are used, respectively [14, 15]. For the decay $\tau \rightarrow \pi K^{0} \overline{K^{0}} \nu_{\tau}$ theoretical estimates are used, which yield a branching ratio of $0.3 \%$ [16].

Two $\tau$ decay analyses are performed according to quasi-exclusive and exclusive classifications. In the quasi-exclusive $\tau$ decay classification $\pi^{0}$ 's are registered as "resolved", "unresolved" or "residual single photon" from $\pi^{0}$. On the other hand, the exclusive classification is more strict as residual single photons are not counted as $\pi^{0}$ 's. The quasi-exclusive analysis 


\begin{tabular}{|c|c|c|}
\hline Class label & $\begin{array}{l}\text { Reconstruction } \\
\text { criteria }\end{array}$ & Generated $\tau$ decay \\
\hline$e$ & $1 e$ & $\tau \rightarrow e^{-\overline{\nu_{e}} \nu_{\tau}}$ \\
\hline$\mu$ & $1 \mu$ & $\tau \rightarrow \mu^{-\overline{\nu_{\mu}}} \nu_{\tau}$ \\
\hline$h$ & $1 h$ & $\begin{array}{cc}\tau \rightarrow \pi^{-} \nu_{\tau} & \tau \rightarrow \pi^{-} K^{0} \overline{K^{0}} \nu_{\tau} \\
\tau \rightarrow K^{-} \nu_{\tau} & \tau \rightarrow K^{-} K^{0} \nu_{\tau} \\
\tau \rightarrow K^{*} \nu_{\tau} & \end{array}$ \\
\hline$h \pi^{0}$ & $1 h+\pi^{0}$ & $\begin{aligned} \tau \rightarrow \rho^{-} \nu_{\tau} & \tau \rightarrow K^{-} \pi^{0} K^{0} \nu_{\tau} \\
\tau \rightarrow \pi^{-} \pi^{0} \frac{K^{0}}{\nu_{\tau}} & \tau \rightarrow K^{*^{-}} \nu_{\tau}\end{aligned}$ \\
\hline$h 2 \pi^{0}$ & $1 h+2 \pi^{0}$ & $\begin{array}{cc}\tau \rightarrow a_{1}^{-} \nu_{\tau} & \tau \rightarrow \pi^{-} \omega \nu_{\tau}^{(1)} \\
\tau \rightarrow K^{*^{-}} \nu_{\tau} & \tau \rightarrow \pi^{-} K^{0} \frac{K^{0}}{\nu_{\tau}} \\
\tau \rightarrow K^{-} 2 \pi^{0} \nu_{\tau} & \tau \rightarrow K^{-} K^{0} \nu_{\tau}\end{array}$ \\
\hline$h 3 \pi^{0}$ & $1 h+3 \pi^{0}$ & $\begin{array}{cl}\tau \rightarrow \pi^{-} 3 \pi^{0} \nu_{\tau} & \tau \rightarrow K^{-} \pi^{0} K^{0} \nu_{\tau} \\
\tau \rightarrow \pi^{-} \pi^{0} \overline{K^{0}} \nu_{\tau} & \tau \rightarrow \pi^{-} \pi^{0} \eta \nu_{\tau}^{(2)}\end{array}$ \\
\hline$h 4 \pi^{0}$ & $1 h+\geq 4 \pi^{0}$ & $\begin{aligned} \tau & \rightarrow \pi^{-4} \pi^{0} \nu_{\tau} \\
\tau & \rightarrow \pi^{-} K^{0} \overline{K^{0}} \nu_{\tau}\end{aligned}$ \\
\hline $3 h$ & $2-4 h$ & $\begin{array}{cc}\tau \rightarrow a_{1}^{-} \nu_{\tau} & \tau \rightarrow K^{-} K^{+} \pi^{-} \nu_{\tau} \\
\tau \rightarrow K^{*-} \nu_{\tau} & \tau \rightarrow \pi^{-} K^{0} \overline{K^{0}} \nu_{\tau} \\
\tau \rightarrow K^{-} \pi^{+} \pi^{-} \nu_{\tau} & \tau \rightarrow K^{-} K^{0} \nu_{\tau}\end{array}$ \\
\hline $3 h \pi^{0}$ & $2-4 h+\pi^{0}$ & $\begin{aligned} \tau & \rightarrow 2 \pi^{-} \pi^{+} \pi^{0} \nu_{\tau}^{(3)} \\
& \tau \rightarrow \pi^{-} \pi^{0} \overline{K^{0}} \nu_{\tau}\end{aligned} \quad \tau \rightarrow K^{-} \pi^{0} K^{0} \nu_{\tau}$ \\
\hline $3 h 2 \pi^{0}$ & $2-4 h+2 \pi^{0}$ & $\begin{aligned} \tau & \rightarrow 2 \pi^{-} \pi^{+} 2 \pi^{0} \nu_{\tau}{ }^{(4)} \\
\tau & \rightarrow \pi^{-} K^{0} \overline{K^{0}} \nu_{\tau}\end{aligned}$ \\
\hline $3 h 3 \pi^{0}$ & $2-4 h+\geq 3 \pi^{0}$ & $\tau \rightarrow 2 \pi^{-} \pi^{+} 3 \pi^{0} \nu_{\tau}$ \\
\hline $5 h$ & $5 h$ & $\tau \rightarrow 3 \pi^{-} 2 \pi^{+} \nu_{\tau}$ \\
\hline $5 h \pi^{0}$ & $5 h+\pi^{0}$ & $\tau \rightarrow 3 \pi^{-} 2 \pi^{+} \pi^{0} \nu_{\tau}$ \\
\hline $\begin{array}{l}\text { Single } \\
\text { photon }\end{array}$ & $\geq 1 h+\geq 0 \pi^{0}+\geq 1 \gamma$ & $\tau \rightarrow \pi^{-} \pi^{0} \eta \nu_{\tau}{ }^{(2,5)}$ \\
\hline
\end{tabular}

${ }^{1}$ With $\omega \rightarrow \pi^{0} \gamma$

${ }^{2}$ With $\eta \rightarrow \gamma \gamma$

3 This channel includes $\tau \rightarrow \pi \omega \nu_{\tau}$ with $\omega \rightarrow \pi^{-} \pi^{+} \pi^{0}$

${ }^{4}$ This channel includes $\tau \rightarrow \pi \pi^{0} \omega \nu_{\tau}$ with $\omega \rightarrow \pi^{-} \pi^{+} \pi^{0}$

${ }^{5}$ With $\eta \rightarrow \pi^{-} \pi^{+} \gamma$

Table 3: Definition of the reconstructed quasi-exclusive $\tau$ decay categories. All $\tau$ decay modes implemented in the simulation are specified for each class. In addition, the single photon class used in the exclusive classification is defined. The notation $\tau$ stands for $\tau^{-}$and the charge conjugate states are implied. 


\begin{tabular}{|c|c|c|c|c|c|c|c|c|c|c|c|c|c|}
\hline Mode & $e$ & $\mu$ & $h$ & $h \pi^{0}$ & $h 2 \pi^{0}$ & $h 3 \pi^{0}$ & $h 4 \pi^{0}$ & $3 h$ & $3 h \pi^{0}$ & $3 h 2 \pi^{0}$ & $3 h 3 \pi^{0}$ & $5 h$ & $5 h \pi^{0}$ \\
\hline$e$ & $\begin{array}{r}72.08 \\
\pm 0.12\end{array}$ & $\begin{array}{c}0.01 \\
\pm 0.01\end{array}$ & $\begin{array}{c}0.49 \\
\pm 0.02\end{array}$ & $\begin{array}{c}0.29 \\
\pm 0.01\end{array}$ & $\begin{array}{c}0.30 \\
\pm 0.02\end{array}$ & $\begin{array}{c}0.19 \\
\pm 0.04\end{array}$ & 0. & $\begin{array}{c}0.01 \\
\pm 0.01\end{array}$ & $\begin{array}{c}0.01 \\
\pm 0.01\end{array}$ & $\begin{array}{c}0.02 \\
\pm 0.02\end{array}$ & 0. & 0. & 0. \\
\hline$\mu$ & 0. & $\begin{array}{r}74.74 \\
\pm 0.12 \\
\end{array}$ & $\begin{array}{c}0.67 \\
\pm 0.02 \\
\end{array}$ & $\begin{array}{c}0.22 \\
\pm 0.01\end{array}$ & $\begin{array}{c}0.05 \\
\pm 0.01\end{array}$ & $\begin{array}{c}0.19 \\
\pm 0.04 \\
\end{array}$ & 0. & $\begin{array}{c}0.01 \\
\pm 0.01\end{array}$ & 0. & 0. & 0. & 0. & 0. \\
\hline$h$ & $\begin{array}{c}0.33 \\
\pm 0.02\end{array}$ & $\begin{array}{c}0.40 \\
\pm 0.02\end{array}$ & $\begin{array}{l}65.27 \\
\pm 0.15\end{array}$ & $\begin{array}{c}3.56 \\
\pm 0.04\end{array}$ & $\begin{array}{c}0.40 \\
\pm 0.02\end{array}$ & $\begin{array}{c}0.14 \\
\pm 0.03\end{array}$ & 0 . & $\begin{array}{c}1.19 \\
\pm 0.04\end{array}$ & $\begin{array}{c}0.10 \\
\pm 0.02\end{array}$ & $\begin{array}{c}0.04 \\
\pm 0.03\end{array}$ & 0 . & 0 . & 0 . \\
\hline$h \pi^{0}$ & $\begin{array}{c}0.24 \\
\pm 0.01\end{array}$ & $\begin{array}{c}0.09 \\
\pm 0.01\end{array}$ & $\begin{array}{c}4.21 \\
\pm 0.06\end{array}$ & $\begin{array}{l}67.16 \\
\pm 0.10\end{array}$ & $\begin{array}{c}11.3 \\
\pm 0.11\end{array}$ & $\begin{array}{c}2.38 \\
\pm 0.14\end{array}$ & $\begin{array}{c}1.00 \\
\pm 0.45\end{array}$ & $\begin{array}{c}0.82 \\
\pm 0.03\end{array}$ & $\begin{array}{c}1.08 \\
\pm 0.05\end{array}$ & $\begin{array}{c}0.22 \\
\pm 0.07\end{array}$ & $\begin{array}{c}0.10 \\
\pm 0.10\end{array}$ & 0. & 0 . \\
\hline$h 2 \pi^{0}$ & $\begin{array}{c}0.05 \\
\pm 0.01\end{array}$ & $\begin{array}{c}0.01 \\
\pm 0.01\end{array}$ & $\begin{array}{c}0.36 \\
\pm 0.02\end{array}$ & $\begin{array}{c}5.41 \\
\pm 0.05\end{array}$ & $\begin{array}{r}58.06 \\
\pm 0.18\end{array}$ & $\begin{array}{r}22.11 \\
\pm 0.38\end{array}$ & $\begin{array}{c}6.86 \\
\pm 1.27\end{array}$ & $\begin{array}{c}0.14 \\
\pm 0.01\end{array}$ & $\begin{array}{c}0.95 \\
\pm 0.05\end{array}$ & $\begin{array}{c}1.14 \\
\pm 0.15\end{array}$ & $\begin{array}{c}0.67 \\
\pm 0.25\end{array}$ & 0. & $\begin{array}{c}0.24 \\
\pm 0.23\end{array}$ \\
\hline$h 3 \pi^{0}$ & 0. & 0 . & $\begin{array}{c}0.04 \\
\pm 0.01\end{array}$ & $\begin{array}{c}0.31 \\
\pm 0.01\end{array}$ & $\begin{array}{c}6.10 \\
\pm 0.09\end{array}$ & $\begin{array}{l}44.22 \\
\pm 0.46\end{array}$ & $\begin{array}{l}34.45 \\
\pm 2.16\end{array}$ & $\begin{array}{c}0.01 \\
\pm 0.01\end{array}$ & $\begin{array}{c}0.18 \\
\pm 0.02\end{array}$ & $\begin{array}{c}0.82 \\
\pm 0.13\end{array}$ & $\begin{array}{c}0.96 \\
\pm 0.30\end{array}$ & $\begin{array}{c}0.19 \\
\pm 0.19\end{array}$ & 0. \\
\hline$h \geq 4 \pi^{0}$ & 0 . & 0 . & $\begin{array}{c}0.01 \\
\pm 0.01\end{array}$ & $\begin{array}{c}0.02 \\
\pm 0.01\end{array}$ & $\begin{array}{c}0.25 \\
\pm 0.02\end{array}$ & $\begin{array}{c}4.00 \\
\pm 0.18\end{array}$ & $\begin{array}{r}\mathbf{2 5 . 0 1} \\
\pm 1.85\end{array}$ & 0 . & $\begin{array}{c}0.02 \\
\pm 0.01\end{array}$ & $\begin{array}{c}0.02 \\
\pm 0.02\end{array}$ & $\begin{array}{c}0.39 \\
\pm 0.19\end{array}$ & 0 . & 0 . \\
\hline $3 h$ & $\begin{array}{c}0.01 \\
\pm 0.01\end{array}$ & $\begin{array}{c}0.02 \\
\pm 0.01\end{array}$ & $\begin{array}{c}0.26 \\
\pm 0.01\end{array}$ & $\begin{array}{c}0.06 \\
\pm 0.01\end{array}$ & $\begin{array}{c}0.03 \\
\pm 0.01\end{array}$ & $\begin{array}{c}0.01 \\
\pm 0.01\end{array}$ & 0 . & $\begin{array}{r}66.76 \\
\pm 0.17\end{array}$ & $\begin{array}{c}4.87 \\
\pm 0.11 \\
\end{array}$ & $\begin{array}{c}0.88 \\
\pm 0.13\end{array}$ & $\begin{array}{c}0.19 \\
\pm 0.14\end{array}$ & $\begin{array}{c}18.56 \\
\pm 1.7\end{array}$ & $\begin{array}{c}2.09 \\
\pm 0.69\end{array}$ \\
\hline $3 h \pi^{0}$ & $\begin{array}{c}0.01 \\
\pm 0.01\end{array}$ & $\begin{array}{c}0.01 \\
\pm 0.01\end{array}$ & $\begin{array}{c}0.21 \\
\pm 0.01\end{array}$ & $\begin{array}{c}0.46 \\
\pm 0.02\end{array}$ & $\begin{array}{c}0.23 \\
\pm 0.02\end{array}$ & $\begin{array}{c}0.09 \\
\pm 0.03\end{array}$ & 0. & $\begin{array}{c}8.35 \\
\pm 0.10\end{array}$ & $\begin{array}{r}\mathbf{5 9 . 4 6} \\
\pm 0.25\end{array}$ & $\begin{array}{r}12.46 \\
\pm 0.49\end{array}$ & $\begin{array}{c}3.91 \\
\pm 0.60\end{array}$ & $\begin{array}{c}7.08 \\
\pm 1.12\end{array}$ & $\begin{array}{l}15.00 \\
\pm 1.72\end{array}$ \\
\hline $3 h 2 \pi^{0}$ & 0 . & 0 . & $\begin{array}{c}0.09 \\
\pm 0.01\end{array}$ & $\begin{array}{c}0.23 \\
\pm 0.01\end{array}$ & $\begin{array}{c}0.66 \\
\pm 0.03\end{array}$ & $\begin{array}{c}0.48 \\
\pm 0.06\end{array}$ & $\begin{array}{c}0.61 \\
\pm 0.35\end{array}$ & $\begin{array}{c}1.13 \\
\pm 0.04\end{array}$ & $\begin{array}{l}10.57 \\
\pm 0.16\end{array}$ & $\begin{array}{l}49.83 \\
\pm 0.71\end{array}$ & $\begin{array}{r}27.95 \\
\pm 1.39\end{array}$ & $\begin{array}{c}1.32 \\
\pm 0.50\end{array}$ & $\begin{array}{c}7.14 \\
\pm 1.24\end{array}$ \\
\hline $3 h \geq 3 \pi^{0}$ & 0 . & 0 . & $\begin{array}{c}0.06 \\
\pm 0.01\end{array}$ & $\begin{array}{c}0.10 \\
\pm 0.01\end{array}$ & $\begin{array}{c}0.31 \\
\pm 0.02\end{array}$ & $\begin{array}{c}0.95 \\
\pm 0.09\end{array}$ & $\begin{array}{c}1.80 \\
\pm 0.60\end{array}$ & $\begin{array}{c}0.23 \\
\pm 0.02\end{array}$ & $\begin{array}{c}1.35 \\
\pm 0.06\end{array}$ & $\begin{array}{r}10.60 \\
\pm 0.41\end{array}$ & $\begin{array}{r}39.06 \\
\pm 1.50\end{array}$ & $\begin{array}{c}0.96 \\
\pm 0.42\end{array}$ & $\begin{array}{c}1.40 \\
\pm 0.56\end{array}$ \\
\hline $5 h$ & 0. & 0 . & 0. & 0. & 0. & 0. & 0 . & $\begin{array}{c}0.03 \\
\pm 0.01\end{array}$ & $\begin{array}{c}0.01 \\
\pm 0.01\end{array}$ & 0. & 0. & $\begin{array}{c}41.9 \\
\pm 2.16\end{array}$ & $\begin{array}{c}5.08 \\
\pm 1.10\end{array}$ \\
\hline $5 h \pi^{0}$ & 0. & 0. & 0. & 0 . & 0. & 0. & 0. & $\begin{array}{c}0.01 \\
\pm 0.01\end{array}$ & $\begin{array}{c}0.01 \\
\pm 0.01 \\
\end{array}$ & 0. & 0. & $\begin{array}{c}2.86 \\
\pm 0.73 \\
\end{array}$ & $\begin{array}{r}37.21 \\
\pm 2.33 \\
\end{array}$ \\
\hline$\overline{\Sigma\left(\mathcal{E}_{i}\right)}$ & $\begin{array}{l}72.72 \\
\pm 0.12\end{array}$ & $\begin{array}{l}75.28 \\
\pm 0.12\end{array}$ & $\begin{array}{l}71.67 \\
\pm 0.17\end{array}$ & $\begin{array}{l}77.73 \\
\pm 0.12\end{array}$ & $\begin{array}{r}77.69 \\
\pm 0.24\end{array}$ & $\begin{array}{r}74.76 \\
\pm 0.65\end{array}$ & $\begin{array}{c}69.73 \\
\pm 3.22\end{array}$ & $\begin{array}{l}78.69 \\
\pm 0.21\end{array}$ & $\begin{array}{l}78.61 \\
\pm 0.33\end{array}$ & $\begin{array}{r}76.03 \\
\pm 0.99\end{array}$ & $\begin{array}{r}73.23 \\
\pm 2.18\end{array}$ & $\begin{array}{c}72.87 \\
\pm 3.13\end{array}$ & $\begin{array}{r}68.16 \\
\pm 3.46\end{array}$ \\
\hline
\end{tabular}

Table 4: Efficiency matrix for the quasi-exclusive classification of hadronic decays. The efficiencies are expressed in percent with their statistical error only. The generated classes are given in the first row, and the reconstructed classes in the first column.

is presented first and the exclusive one is postponed to Section 10. Table 4 describes the quasi-exclusive efficiency matrix $\mathcal{E}_{i j}$ for the 13 reconstructed categories. The matrix $\mathcal{E}_{i j}$ gives the probability of a $\tau$ decay generated in class $i$ to be reconstructed in class $j$ as given by the Monte Carlo but where the systematic effects related to the pion misidentification probability are already corrected for. The good diagonal behaviour of this matrix can be noticed. The off-diagonal elements become more sizeable as the number of $\pi^{0}$ 's increases. The number of observed events in each class of the data sample is given in Table 5 for the two classifications.

Figure 16 shows the invariant mass for the quasi-exclusive selected $h \pi^{0}, h 2 \pi^{0}$ decays. For the $h 2 \pi^{0}$ invariant mass distribution a good agreement between the simulation and data is observed, despite a slight discrepancy in the invariant mass distributions of the sub-systems $h \pi^{0}$ and $\pi^{0} \pi^{0}$ discussed in Section 9.2.

The invariant mass distributions for the $h 3 \pi^{0}, h 4 \pi^{0}, 3 h$ and $3 h \pi^{0}$ decays are shown in Figure 17 in good agreement with the simulation. For the $3 \mathrm{~h}$ decays, a slight disagreement is

\footnotetext{
${ }^{6}$ For all the invariant mass plots the pion mass is assumed for the charged hadrons unless otherwise stated.
} 


\begin{tabular}{|c|c|c|c|c|}
\hline \multirow{2}{*}{ Mode } & \multicolumn{2}{|c|}{ Quuasi-exclusive } & \multicolumn{2}{c|}{ Exclusive } \\
\cline { 2 - 5 } & $N^{\text {obs }}$ & $N_{\text {non- }}^{\text {exp }}$ & $N^{\text {obs }}$ & $N_{\text {non- } \tau}^{\text {exp }}$ \\
\hline \hline$e$ & 21298 & $237.2 \pm 16$ & 21298 & $237.2 \pm 16$ \\
\hline$\mu$ & 21456 & $188.6 \pm 14$ & 21456 & $188.6 \pm 14$ \\
\hline$h$ & 15083 & $63.4 \pm 8$ & 15083 & $63.4 \pm 8$ \\
\hline$h \pi^{0}$ & 31246 & $78.7 \pm 9$ & 24840 & $58.9 \pm 8$ \\
\hline$h 2 \pi^{0}$ & 12217 & $46.3 \pm 7$ & 5691 & $10.5 \pm 3$ \\
\hline$h 3 \pi^{0}$ & 2270 & $16.8 \pm 4$ & 683 & $5.1 \pm 2$ \\
\hline$h \geq 4 \pi^{0}$ & 232 & $6.8 \pm 3$ & 41 & $1.1 \pm 1$ \\
\hline \hline $3 h$ & 11176 & $65.7 \pm 8$ & 11176 & $65.7 \pm 8$ \\
\hline $3 h \pi^{0}$ & 6156 & $92.1 \pm 10$ & 3897 & $52 . \pm 7$ \\
\hline $3 h 2 \pi^{0}$ & 1806 & $55 . \pm 7$ & 685 & $21.2 \pm 5$ \\
\hline $3 h \geq 3 \pi^{0}$ & 500 & $43.4 \pm 7$ & 172 & $12 . \pm 3$ \\
\hline \hline $5 h$ & 59 & $1.5 \pm 1$ & 59 & $1.5 \pm 1$ \\
\hline $5 h \pi^{0}$ & 33 & $15.4 \pm 4$ & 17 & $11.9 \pm 3$ \\
\hline
\end{tabular}

Table 5: Numbers of selected events in the quasi-exclusive and exclusive $\tau$ decay classifications for 1991-1993 periods.

also found for the invariant mass of the sub-systems with like-sign and unlike-sign particles. In the $3 h \pi^{0}$ decay, the $\omega$ and $\rho$ resonances are observed in a separate analysis [17].

The invariant mass distributions for the $3 h 2 \pi^{0}, 3 h 3 \pi^{0}, 5 h$ and $5 h \pi^{0}$ decays are shown in Figure 18 in agreement with the Monte Carlo expectation. The $3 h 3 \pi^{0}$ final state is dominated by background from other $\tau$ decays because of secondary interactions and fake photons. This results in a weak evidence for a signal, which is at the limit of the sensitivity of the global analysis. Stricter cuts to reduce background yield a consistent signal. In addition to the poor knowledge of the dynamics in the $3 h 3 \pi^{0}$ decay, the invariant mass distribution is affected by systematics effects (given in Section 9) which are not reflected in Figure 18. 

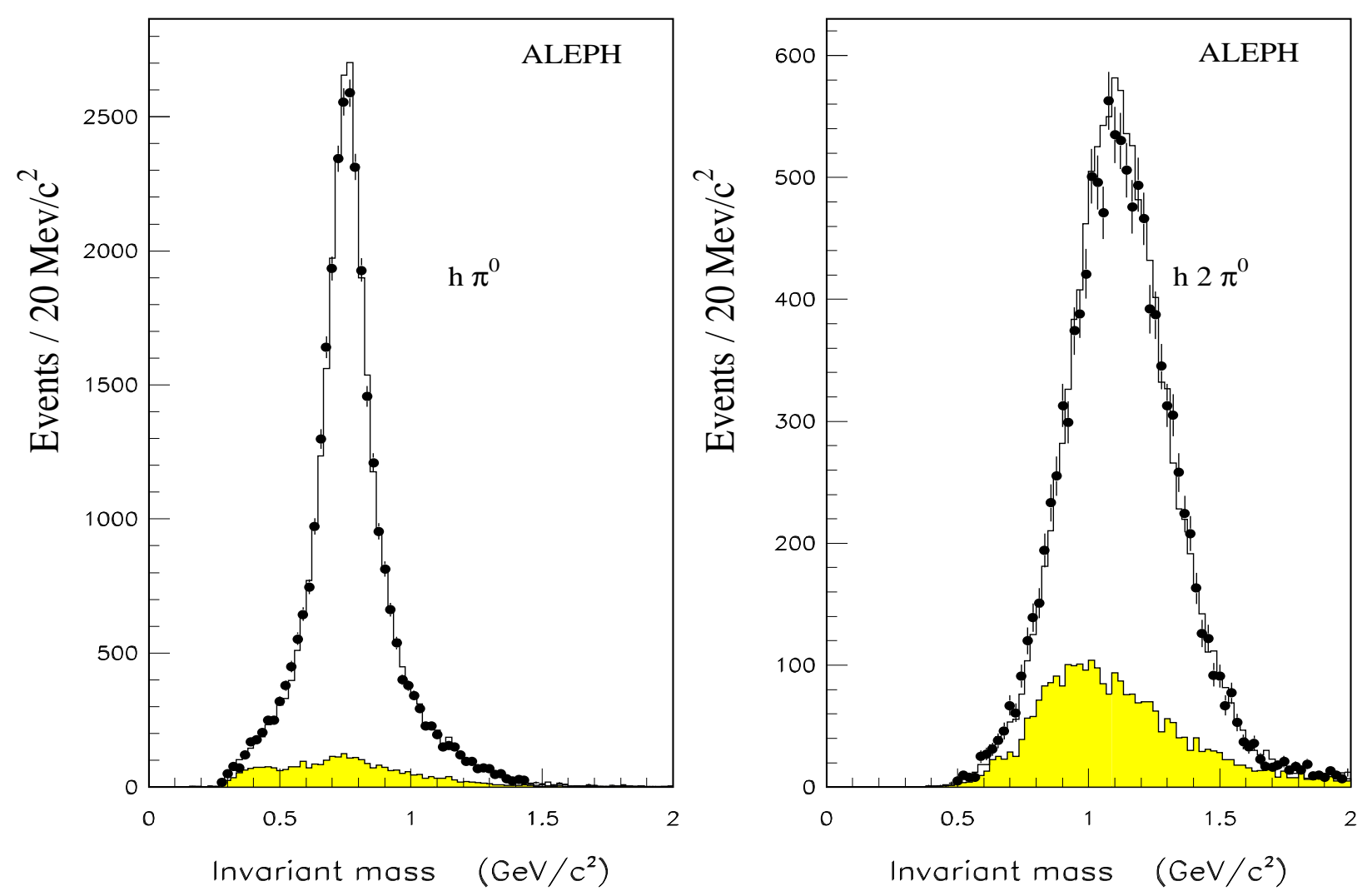

Figure 16: Invariant mass distributions for the $h \pi^{0}$ and $h 2 \pi^{0}$ quasi-exclusive selected samples. The points with error bars show the observed distributions, the solid histograms represent the simulated distributions and the shaded histograms account for the expected $\tau$ background from the Monte Carlo computed with the measured branching ratios. 

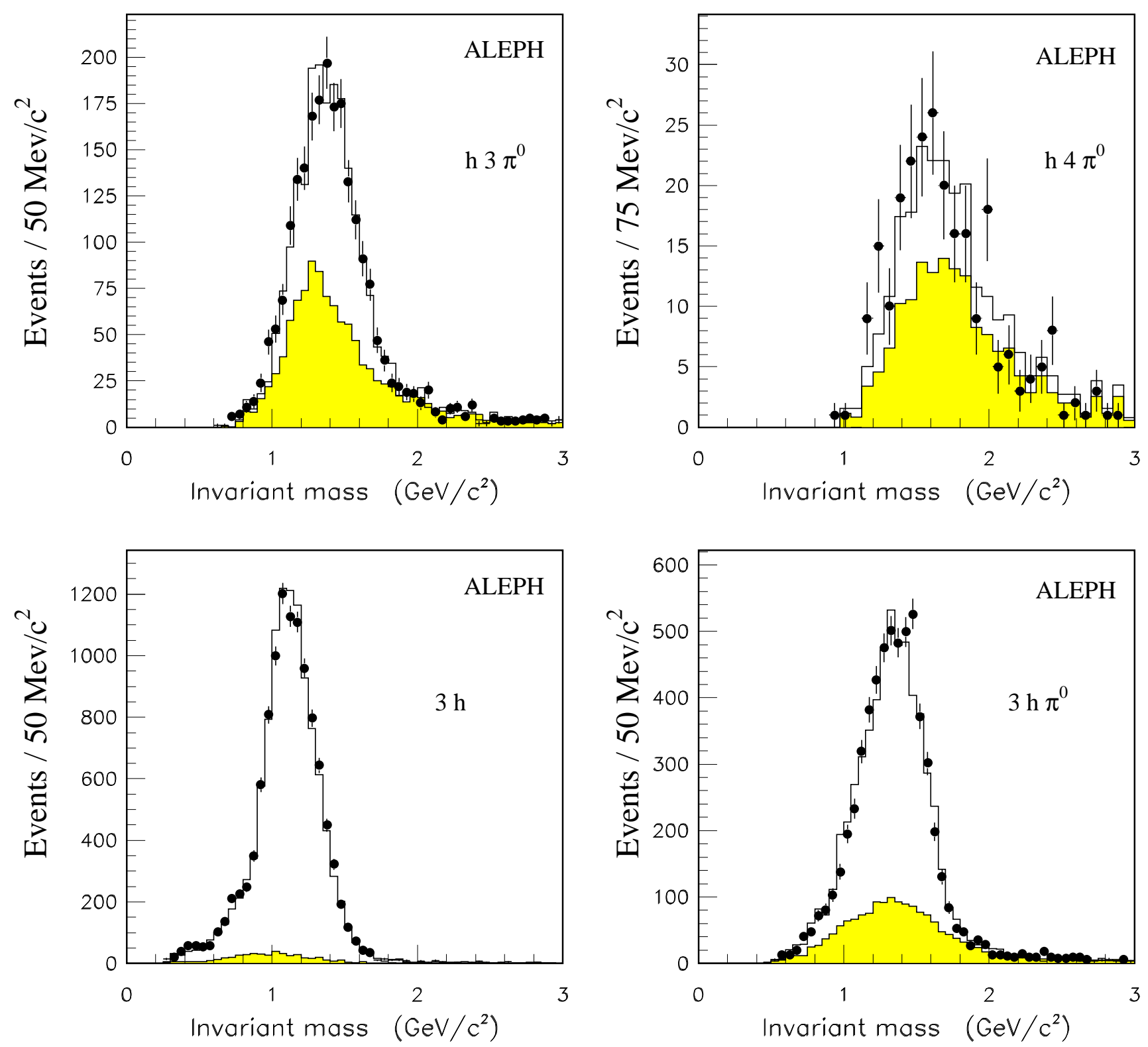

Figure 17: Invariant mass distributions for the $h 3 \pi^{0}, h \geq 4 \pi^{0}, 3 h$ and $3 h \pi^{0}$ quasi-exclusive selected samples. The points with error bars show the observed distributions, the solid histograms represent the simulated distributions and the shaded histograms account for the expected $\tau$ background from the Monte Carlo computed with the measured branching ratios. 

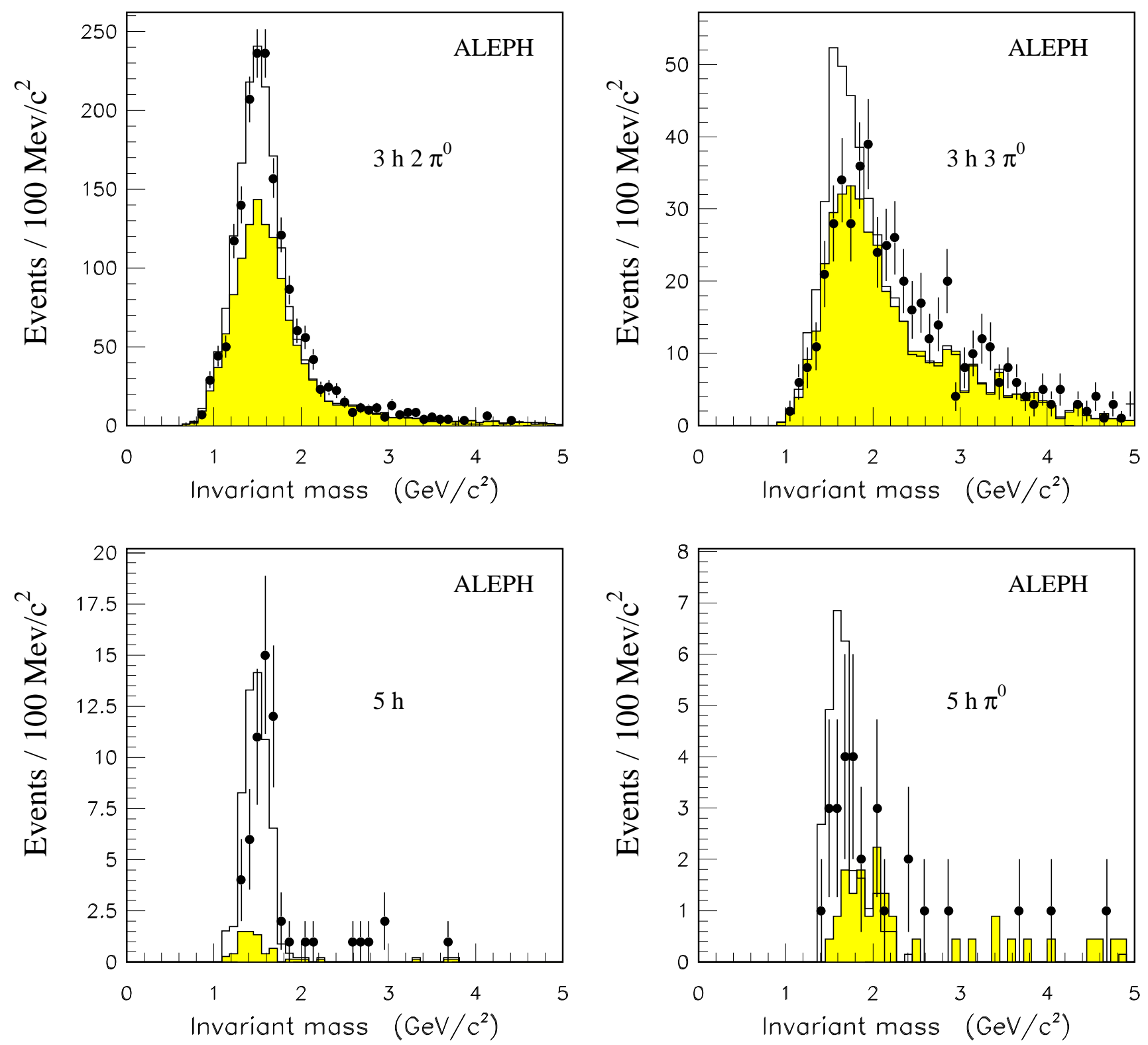

Figure 18: Invariant mass distributions for the $3 h 2 \pi^{0}, 3 h 3 \pi^{0}$, $5 h$ and $5 h \pi^{0}$ quasi-exclusive selected samples. The points with error bars show the observed distributions, the solid histograms represent the simulated distributions and the shaded histograms account for the expected $\tau$ background from the Monte Carlo computed with the measured branching ratios. 


\section{Extraction of branching ratios}

In order to extract a measurement of the $\tau$ branching ratios from this global analysis, the number of observed events in a given class $j$ after the non- $\tau$ background subtraction divided by the sum of all observed events defines the observed fraction of events $f_{j}$ in the class $j$. The fraction $f_{j}$ is related to the branching ratios $B_{i}$ to be determined by the following equation:

$$
f_{j}=\frac{\sum_{i} \mathcal{E}_{i j} B_{i}}{\sum_{i k} \mathcal{E}_{i k} B_{i}},
$$

where $\mathcal{E}_{i j}$ is the efficiency matrix (defined in the previous section). The linear system defined by the $f_{j}$ 's is solved with the additional constraint

$$
\sum_{i} B_{i}=1
$$

For convenience, a maximum likelihood technique is used to solve the system and to estimate the errors.

It should be noticed that the efficiency matrix $\mathcal{E}_{i j}$ is independent of the $\tau$ branching ratios used in the Monte Carlo simulation, except for the subclasses contributing to each defined class as shown in Table 3. The effect depends however on small branching ratios and the procedure used in this correction relies on measured values. In this analysis the branching ratios used for the Cabibbo-suppressed and Cabibbo-allowed decays are consistent with the ALEPH measurements [5, 6].

A correction to the $\mathcal{E}_{i j}$ efficiency matrix is applied to take into account the deficit of $16 \%$ of fake photons in the simulation. This correction is computed as the opposite of the effect obtained in the efficiency matrix when the number of fake photons in the Monte Carlo is reduced by $16 \%$. This linear procedure is justified from studies made with the Monte Carlo. As a check, a model producing this excess of fake photons was implemented in the simulation and the complete $\pi^{0}$ reconstruction procedure was repeated. The two methods yield consistent results.

The analysis relies on the standard $\tau$ decay description [10]. One could imagine unknown decay modes not included in the simulation but since large efficiencies are achieved in the $\tau$ selection it is difficult to overlook them in the analysis. A notable exception could be low momentum particles coming from decays like $\tau \rightarrow e N^{0}$ with $m_{N^{0}} \sim m_{\tau}$ and $N^{0}$ not decaying in the detector volume, so it is important to make sure that such decays are not present. An independent measurement of the branching ratio for undetected modes by a direct search was done in ALEPH [18], limiting this branching ratio to less than $0.11 \%$ at $95 \%$ CL and justifying the assumption that the sum of the branching ratios for visible $\tau$ decays is equal to one. 


\section{Systematic uncertainties}

The possible sources of systematic uncertainties come mainly from the particle identification, the selection of $\tau$ pair events, and the photon and $\pi^{0}$ reconstruction. The systematics on the particle identification and $\tau$ event selection are described in detail in reference [4]. For the $\tau$ hadronic decay modes reported in this paper special emphasis is given to the systematics affecting the $\pi^{0}$ reconstruction. In most cases, possible deviations in the Monte Carlo simulation compared to the data are propagated to the efficiency matrix and a new set of branching ratios is derived. The differences with the reference values or the statistical error of the test are quoted as systematic errors. With this procedure, the correlations among the several classes are also taken into account.

\subsection{Photon and $\pi^{0}$ reconstruction}

The relevant sources of systematic effects in the photon and $\pi^{0}$ reconstruction are described in the following. First, the systematics associated with the photon clustering algorithm are examined.

- The inefficiency of the clustering algorithm described in Section 5.2 is directly studied with an independent sample of electrons from $\tau$ decays. In this study the cut of the distance between the electromagnetic cluster and the charged track is removed, so electron showers are considered as photon candidates. Because of the magnetic bending this method only covers the domain above $1 \mathrm{GeV}$. This study shows that the efficiency is well described by the simulation with a ratio of data over Monte Carlo essentially energy independent, differing from unity by $(0.23 \pm 0.15) \%$. The column with the $\mathcal{E}_{\text {clust }}$ label shows the derived systematics in Table 6 .

- In the same table the systematic effect from a global energy scale (labeled as $E_{\text {scale }}$ ) is given. The uncertainty in the global energy scale error for the ECAL pad calibration is taken to be $0.3 \% \oplus 3 \% / \sqrt{E}$ (with $\mathrm{E}$ in $\mathrm{GeV}$ ) from imperfect pad clustering corrections [19].

- The spectrum of low energy photons belonging to resolved $\pi^{0}$ 's is given in Figure 19 after subtraction of the fake photon contribution affecting this sample. The systematics related to the fake photons are discussed below. The photon efficiency is fairly well reproduced by the simulation, in particular in the region below $1 \mathrm{GeV}$ which was not tested with electrons using the previous method. From the comparison in this region the inefficiency for photons in the data is larger by $(4.4 \pm 3.4) \%$ with respect to the Monte Carlo. This correction is applied in the analysis. Changing the relative efficiency between data and Monte Carlo by $3.4 \%$ yields the systematic uncertainties shown in 


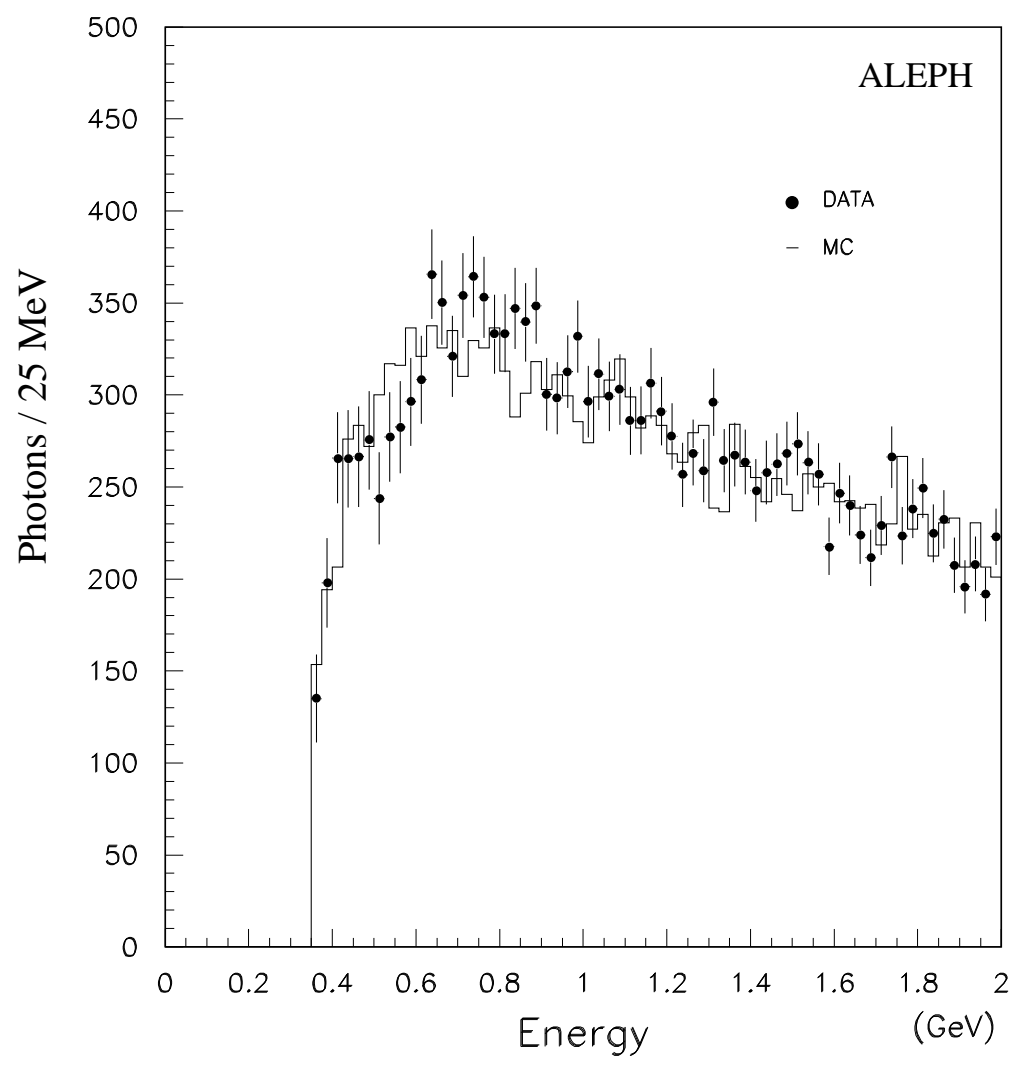

Figure 19: The points with error bars show the energy spectra below $2 \mathrm{GeV}$ for the reconstructed photons entering resolved $\pi^{0}$ 's and the solid histogram from simulation, after subtraction of fake photons.

Table 6 in the column labeled as $\mathcal{E}_{\text {low }}$. This error is equivalent to a shift of $20 \mathrm{MeV}$ in the effective threshold for $\pi^{0}$ reconstruction.

- The minimal distance between the barycentre of an electromagnetic cluster and the closest charged track in the same hemisphere is an important parameter to veto fake photon candidates from the hadron interactions in ECAL at the expense of some inefficiency for the signal ( $\sim 3 \%$ of the photons). The simulation of the efficiency for reconstructing genuine photons in the close environment of a charged hadron relies on the description of hadronic and electromagnetic showers in the Monte Carlo. If the photon shower is not identified as a separate cluster, it is expected that the barycentre of the compound shower will be shifted from the track impact point and the cluster will be registered as a photon candidate if the distance exceeds the $2 \mathrm{~cm}$ cut value. The distribution of this distance as given in Figure 20 for all photons reconstructed as genuine shows a step rise above the minimum value in agreement with the simulation (the fact that a few photons appear with a value below the cut is explained in Section 5.2). The raw distribution for photons at the Monte Carlo generator level is given for comparison, 


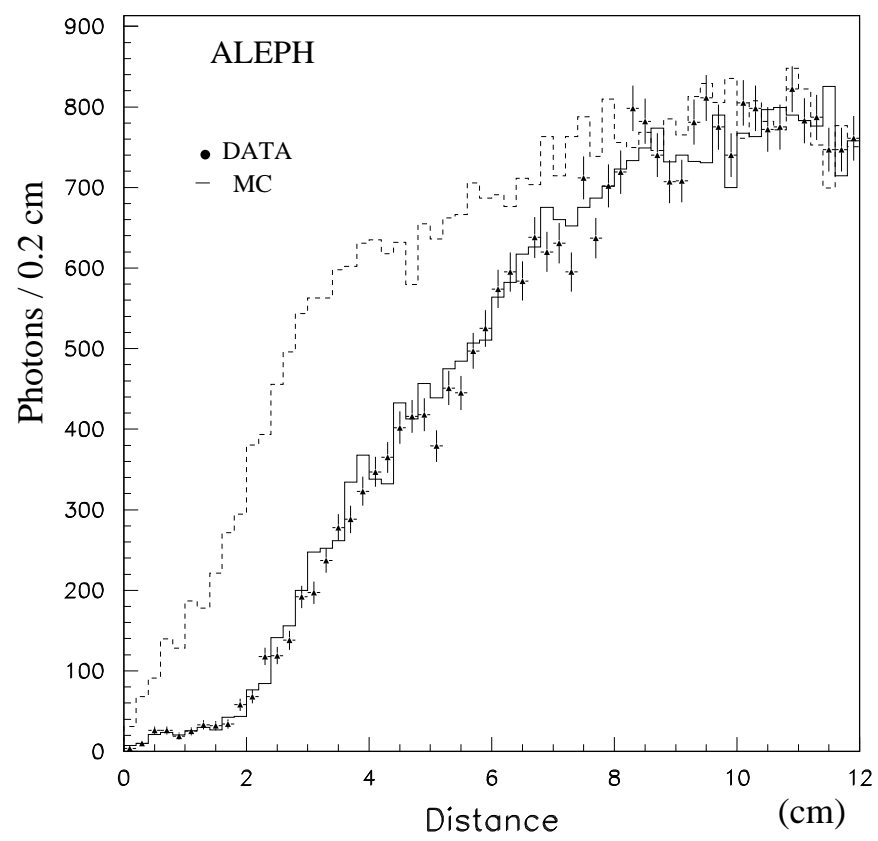

Figure 20: The distribution of the distance between the barycentre of a reconstructed photon from a $\pi^{0}$ and the closest charged track is given as points with error bars. The solid histogram shows the Monte Carlo simulation and the dotted histogram corresponds to the distribution at the generator level. Both Monte Carlo histograms are normalized for distances larger than $10 \mathrm{~cm}$.

showing the inefficiency associated to the hadronic environment in the electromagnetic calorimeter. The efficiencies can be directly compared for distances less than $8 \mathrm{~cm}$ and the systematic uncertainty is derived from the statistical error of the comparison. The possible disagreement between data and Monte Carlo can be effectively described by a change in the cut value by less than $1 \mathrm{~mm}$. The corresponding systematic uncertainties appear in Table 6 labeled as $d_{\gamma t}$.

- The variables that define a pair of charged tracks coming from a conversion, such as the invariant mass and the distance in the plane transverse to the beam between the two tracks at the point of closest approach, show a good agreement between data and Monte Carlo. The effect of the misidentification probabilities in the particle identification quoted in Section 4 is found to be negligible. The difference in the fraction of converted photons mentioned in Section 5.1 (which could produce a bias through the difference of efficiencies for converted and non-converted photons) also induces a negligible systematic uncertainty.

Next, the systematics of the photon likelihood identification are described. 


\begin{tabular}{|c|c|c|c|c|c|c|c|c|c|}
\hline Mode & $\mathcal{E}_{\text {low }}$ & $d_{\gamma t}$ & $\mathcal{E}_{\text {clust }}$ & $E_{\text {вcale }}$ & ref & $D^{\pi^{0}}$ & $m_{\pi^{0}}$ & fake & $\sigma_{\pi^{0}}^{\text {Total }}$ \\
\hline \hline$h$ & 0.027 & 0.034 & 0.032 & 0.026 & 0.033 & 0.010 & 0.006 & 0.060 & 0.097 \\
\hline$h \pi^{0}$ & 0.035 & 0.010 & 0.006 & 0.042 & 0.048 & 0.010 & 0.023 & 0.040 & 0.088 \\
\hline$h 2 \pi^{0}$ & 0.022 & 0.026 & 0.020 & 0.023 & 0.032 & 0.010 & 0.025 & 0.050 & 0.082 \\
\hline$h 3 \pi^{0}$ & 0.043 & 0.019 & 0.011 & 0.065 & 0.049 & 0.011 & 0.008 & 0.040 & 0.104 \\
\hline$h \geq 4 \pi^{0}$ & 0.041 & 0.020 & 0.010 & 0.065 & 0.013 & 0.011 & 0.008 & 0.020 & 0.085 \\
\hline $3 h$ & 0.032 & 0.033 & 0.007 & 0.030 & 0.016 & 0.004 & - & 0.048 & 0.075 \\
\hline $3 h \pi^{0}$ & 0.032 & 0.013 & 0.002 & 0.025 & 0.010 & 0.004 & 0.002 & 0.025 & 0.051 \\
\hline $3 h 2 \pi^{0}$ & 0.016 & 0.011 & 0.003 & 0.032 & 0.023 & 0.008 & 0.005 & 0.050 & 0.067 \\
\hline $3 h \geq 3 \pi^{0}$ & 0.015 & 0.010 & 0.008 & 0.026 & 0.004 & 0.005 & 0.006 & 0.019 & 0.040 \\
\hline $5 h$ & 0.008 & 0.001 & - & 0.002 & 0.004 & 0.002 & - & 0.001 & 0.010 \\
\hline $5 h \pi^{0}$ & 0.007 & 0.001 & - & 0.001 & 0.004 & 0.002 & - & 0.002 & 0.008 \\
\hline
\end{tabular}

Table 6: Systematic effects affecting the $\pi^{0}$ reconstruction in the quasi-exclusive classification. All these values are absolute (branching ratios in \%). Symbols are defined in the text.

- To check the likelihood method, which uses different estimators to tag the genuine and fake photons, a careful study of the reference distributions of the probability densities is needed. The simulation of hadronic interactions in the electromagnetic calorimeter is studied in a selected sample of pions in data and in Monte Carlo. A resonable description of the hadronic showers in the simulation is found, although slight discrepancies concerning the modeling of fluctuations of hadronic showers are observed as seen in Figure 5. The simulation of fake photons from electromagnetic showers presents as well some differences as shown in the $d_{\gamma \gamma}$ distribution in Figure 5. Different correction functions are evaluated with data and their uncertainties are propagated to the corresponding estimators as stated in Section 5.3 taking the correlations into account. The maximal effect observed in the branching ratios is given in Table 6 under the column label ref by switching on and off such corrections.

- An excess of fake photons is observed in data with respect to the Monte Carlo. This excess is measured to be $16 \%$ in the data by comparing the relative weight of the Monte Carlo distributions for genuine and fake photons to the data distributions. This excess is corrected in the extraction of the branching ratios as described in Section 8. The correction corresponds to an average excess of fake photons, but does not take into account the relative contribution of electromagnetic and hadronic fake photons expected to vary from channel to channel. Thus, a systematic uncertainty is assigned to each channel by comparing the distributions of the $P_{\gamma \text { Res }}$ estimator of the rejected photons for data and Monte Carlo. The relative number of rejected fake photons and the shape 
of the respective distributions provide an estimate of the loss of events in this channel due to unrecognized fake photons. This procedure yields systematic uncertainties which are about half the full correction applied. The results are quoted in the column labeled as fake in Table 6 .

Finally, the systematics associated with the $\pi^{0}$ procedure are discussed.

- In order to study the systematic effects affecting the $\pi^{0}$ reconstruction, different parametrizations are used for the resolutions and the effective mass needed in the computation of $P_{\pi^{0}}$ for $\pi^{0}$ 's with and without converted photons. The derived systematic uncertainty is shown under label $m_{\pi^{0}}$.

- The estimator for a pair of photons to originate from a $\pi^{0}, D_{i, j}^{\pi^{0}}$, seems to indicate an excess of data in the first bins (Figure 8). This indication can be interpreted as scale factor between data and Monte Carlo distributions, corresponding to a shift around the cut value which is found to be $0.00017 \pm 0.00009$ by a $\chi^{2}$ minimization. The derived systematic effect when applying the shift measured in the data is given in Table 6 under the column label $D^{\pi^{0}}$.

Finally, in the last column in Table 6 the total error $\sigma_{\pi^{0}}^{\text {total }}$ from the $\pi^{0}$ reconstruction is computed as the quadratic sum of all components.

\subsection{Tau selection and particle identification}

As explained in Section 3, the overall $\tau$ pair selection efficiency at the $Z^{0}$ peak is measured on $\tau$ data directly and is found to be $(78.84 \pm 0.13) \%$. The corresponding value for the Monte Carlo is $(78.99 \pm 0.09) \%$, which is in agreement with the previous value. Because of the known discrepancies between Monte Carlo and data concerning the simulation of fake showers in the electromagnetic and hadronic calorimeters, it is important to test that the $\tau$ pair selection does not contain a channel-dependent efficiency not reproduced by the Monte Carlo simulation. Such a hypothetical dependence would induce, in principle, a bias in the efficiency matrix. With a version of the $\tau$ selection designed to be more sensitive to such fake showers by removing the energy thresholds for neutral objects, the global branching ratio analysis is repeated so that a systematic uncertainty is quoted for every channel. Table 7 shows the derived value in the column $\tau$ - sel.

As $16 \%$ of the data sample in this analysis corresponds to off-peak energies, the Monte Carlo simulation is used to determine the drop of $\tau$ selection efficiencies, which is found to be $1.7 \%$ and $0.8 \%$ at + and $-2 \mathrm{GeV}$ of the peak energy, respectively. This is due to initial state radiation and no channel-dependent effect is observed. 


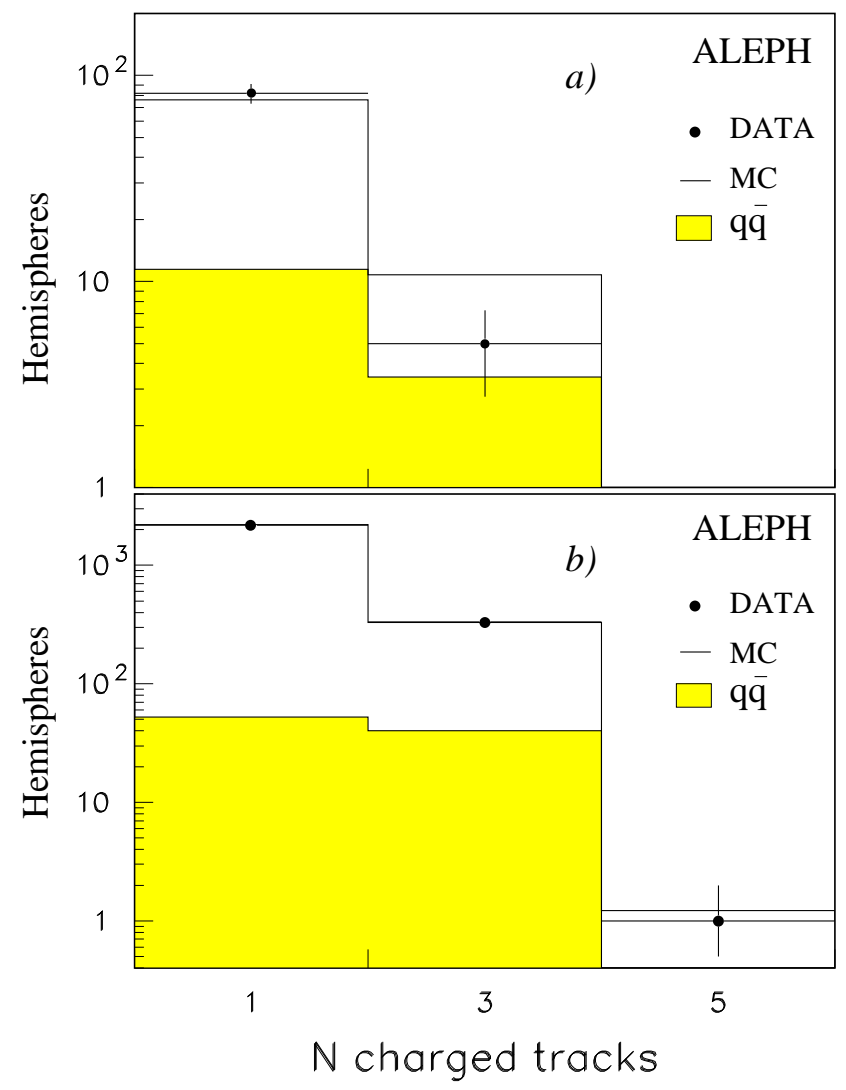

Figure 21: Distribution of charged tracks in the opposite hemispheres in which five charged hadrons are selected (figure a) and five hadrons at least (any combination of charged and neutral hadrons, figure b).

The uncertainties on the contribution of non- $\tau$ background are now considered. In spite of the non- $\tau$ background being less than one percent over the whole sample, its distribution is not uniform in the different reconstructed classes. In particular, the $q \bar{q}$ background should affect primarily the classes of high hadron multiplicity. In Figure 21 the distribution of charged tracks in the opposite hemispheres in which five hadrons are selected is shown with the Monte Carlo expectations and a good agreement is observed. A maximal variation of $\pm 50 \%$ for this background in each channel leads to a small contribution which is included in the final systematic error.

Of particular interest is the electron and muon contamination in the one-prong hadron sample, which is known from other studies not to be completely reproduced by the Monte Carlo. However, a procedure is developed using data to actually measure the relevant efficiencies in each data sample [4]. The quoted systematics for such effects are given in the column labeled as $p-i d$ of Table 7 . 


\subsection{Tracking and secondary interactions}

The tracking uncertainties contribute differently according to the topology. For the oneprong hemispheres, they do not enter significantly since no absolute efficiency is needed in the global analysis. In the three-prong decays, their effect is strongly reduced since 2, 3, and 4 charged tracks are accepted in the three-prong definition. Possible uncertainties in the track reconstruction coming either from the sharing of coordinate points in the multi-track environment or from the description of $K_{S}^{0} \rightarrow \pi^{-} \pi^{+}$decays are also investigated since the feed-across between one- and three-prongs could be affected. In a specific analysis dealing with the $K_{S}^{0}$ reconstruction, no significant discrepancy with respect to the simulation is observed. Analyzing $\tau$ pair events in which the two hemispheres have like-sign charges in Monte Carlo and in data allows to test the probability to lose the two like-sign particles in a three-prong hemisphere. According to the Monte Carlo about half of the sample of like-sign hemispheres originates from three-prong decays if a strong cut on the track association to the interaction point $\left(d_{0}\right)$ is applied; a total of $291 \pm 17$ events are observed in data in agreement with $269 \pm 12$ expected by the Monte Carlo simulation. The proportion of two charged tracks in the three-prong hemispheres is also observed to be higher in the data by $(7.6 \pm 4.5) \%$. These measurements are interpreted in terms of tracking inefficiency and are used to compute the quoted systematic errors given in the column labeled tracking.

Secondary interactions of hadrons in the detector can affect the reconstructed topology of final states for both charged particles and $\pi^{0}$ s. The effect on charged particles depends on their momenta and the radial distance at which the interactions takes place because of the $d_{0}$ cut used to define a good track. It is important to test the simulation of interactions in the Monte Carlo and assess the consequences on the feedthrough of events between different $\tau$ decays classes.

The basic ingredient used for this analysis is the $d_{0}$ distribution of charged tracks for each topology. Here $d_{0}$ is given a sign from the track angular momentum at the point of closest approach to the beam crossing point in the plane transverse to the beam direction. In this way, the sign of $d_{0}$ is equal to the charge of a secondary track collinear to the interacting hadron at the production point. Figure 22 shows such distributions for one and three-prong decays. Primary tracks from the beam-beam interaction point present a sharp $d_{0}$ peak with a fall-off which is underestimated by the simulation. This has no consequence for this analysis which uses a wide $d_{0}$ cut at $2 \mathrm{~cm}$. Beyond this peak, long tails are observed which are well explained by the simulation as originating from secondary interactions and tracking problems in a multi-track environment (even in one-prong hemispheres about $20 \%$ of the decays have at least one additional track from photon conversions). Note the expected excess of positive secondary tracks reproduced by the simulation. The agreement between data and Monte Carlo can be expressed as the ratio of events for $\left|d_{0}\right|$ larger than $0.5 \mathrm{~cm}$ which is $1.004 \pm 0.045$ and $0.983 \pm 0.031$ for one and three-prong decays, respectively. The average interaction probability for a track in the sample is $2.3 \%$ from the simulation, mostly at small $d_{0}$ values not affecting significantly the topology. Agreement is observed for the tails of the $d_{0}$ distributions 


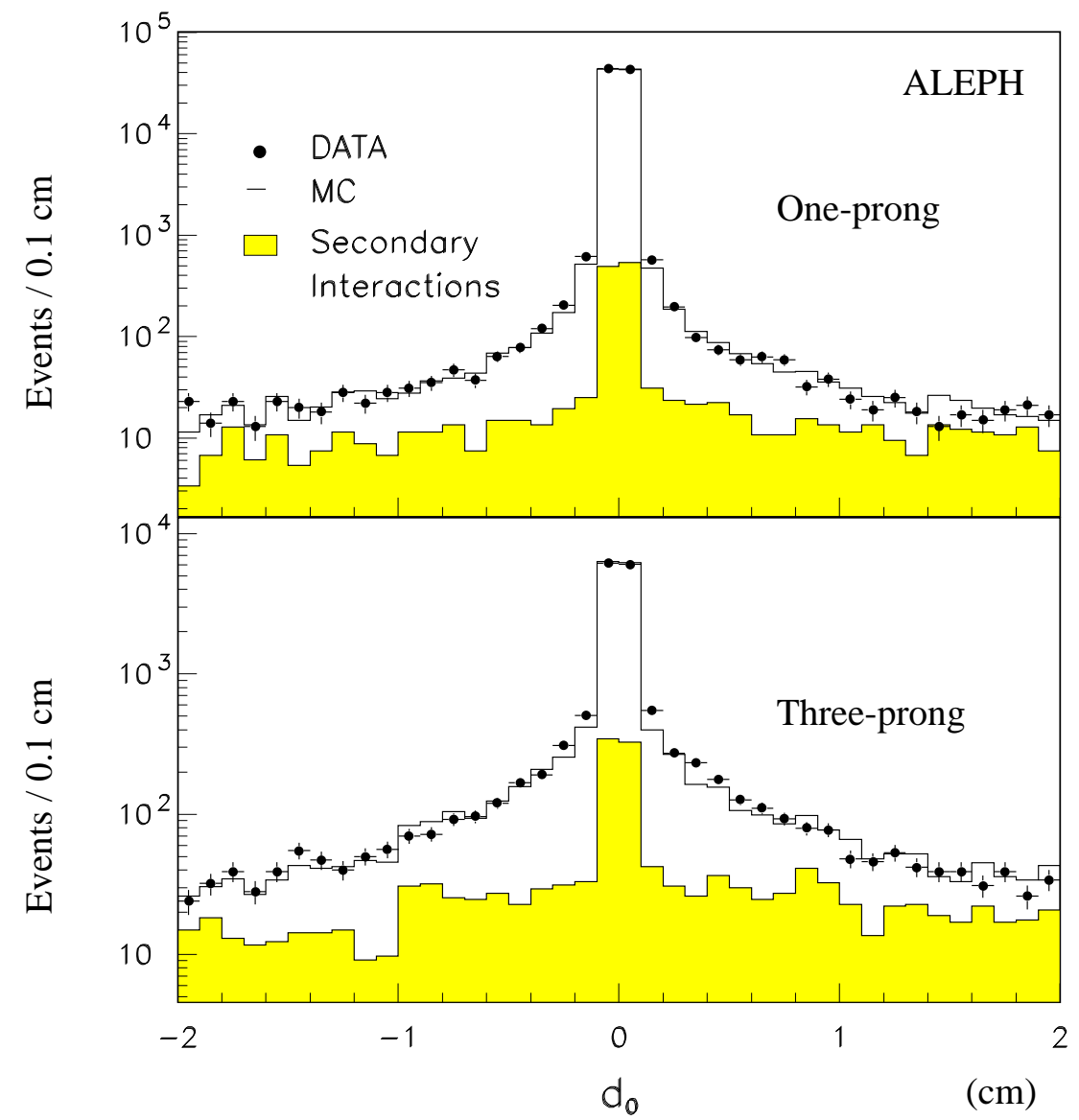

Figure 22: $d_{0}$ distribution for one and three-prong hemispheres. The points with error bars show the data and the solid histogram the Monte Carlo. The shaded histograms correspond to the secondary interactions from the simulation. For the three-prong decays only the largest $d_{0}$ value is plotted.

in data and Monte Carlo for all hadron topologies, checking the simulation of charged tracks and $\pi^{0}$ 's in secondary interactions. From this comparison, systematic uncertainties on the branching ratios are quoted in Table 7 under the heading inter.

\subsection{Tau decay dynamics}

Uncertainties in the dynamics of the hadronic $\tau$ decays can lead to systematics effects when computing the efficiency matrix. Studying the efficiencies as a function of hadronic mass checks for possible bias depending on the resonance structure in the hadronic final state. These checks are performed for all the $\tau$ decay categories. In the $h \pi^{0}$ final state a good description of the $\rho-\rho^{\prime}$ interference is found, and no dependence on the $h \pi^{0}$ invariant mass is observed for the efficiency. Alternative models for the description of the dynamics of the 
$a_{1}$ resonance have been proposed in reference [20]. The associated systematic effect due to the uncertainty on the ratio of $\mathrm{D}$ to $\mathrm{S}$ waves $[21,22]$ is computed through a reweighting of the Monte Carlo matrix elements. Also, $\tau$ decays in the $3 h 2 \pi^{0}$ final state were generated with a non-resonant amplitude. The new version of the generator [23] now includes the resonant channel $\pi \pi^{0} \omega$ which dominates this class, as reported by CLEO [12]. Dedicated Monte Carlo productions allow to correct these efficiencies for the involved channels. The estimated systematic uncertainties are quoted in the column labeled $\varepsilon_{D y n}$.

The efficiency matrices for right-handed and left-handed $\tau$ 's are computed separately from the simulation. Using the $\tau$ polarization measured by ALEPH [19] within the experimental uncertainty, the resulting efficiencies are evaluated. The effect on the branching ratios is found to be small, and it is included in the final systematic errors.

\subsection{Summary on systematic uncertainties}

\begin{tabular}{|c|c|c|c|c|c|c|c|c|}
\hline Mode & $\sigma_{\pi^{0}}^{\text {total }}$ & $\sigma_{\tau-\text { sel }}$ & $\mathrm{p}-\mathrm{id}$ & tracking & inter & $\varepsilon_{\text {Dyn }}$ & $M C_{\text {stat }}$ & $\sigma_{\text {sys }}^{\text {total }}$ \\
\hline \hline$h$ & 0.097 & 0.029 & 0.019 & - & 0.014 & - & 0.036 & 0.110 \\
\hline$h \pi^{0}$ & 0.088 & 0.014 & 0.017 & - & 0.022 & - & 0.051 & 0.106 \\
\hline$h 2 \pi^{0}$ & 0.082 & 0.017 & 0.003 & - & 0.010 & 0.040 & 0.045 & 0.104 \\
\hline$h 3 \pi^{0}$ & 0.104 & 0.019 & - & - & 0.015 & 0.008 & 0.033 & 0.112 \\
\hline$h \geq 4 \pi^{0}$ & 0.085 & 0.006 & - & - & 0.011 & - & 0.017 & 0.088 \\
\hline $3 h$ & 0.075 & 0.014 & - & 0.020 & 0.026 & 0.006 & 0.028 & 0.088 \\
\hline $3 h \pi^{0}$ & 0.051 & 0.034 & - & 0.009 & 0.021 & 0.007 & 0.031 & 0.073 \\
\hline $3 h 2 \pi^{0}$ & 0.067 & 0.011 & - & 0.001 & 0.009 & 0.012 & 0.025 & 0.074 \\
\hline $3 h \geq 3 \pi^{0}$ & 0.040 & 0.013 & - & - & 0.007 & 0.010 & 0.016 & 0.047 \\
\hline $5 h$ & 0.010 & 0.004 & - & 0.004 & 0.005 & - & 0.004 & 0.013 \\
\hline $5 h \pi^{0}$ & 0.008 & 0.003 & - & 0.001 & 0.002 & - & 0.003 & 0.012 \\
\hline
\end{tabular}

Table 7: Summary of systematics errors for the branching ratios obtained from the quasiexclusive classification. Dashes denote negligible contributions. All these values are absolute (branching ratios in \%). Symbols are defined in the text.

In the last column of Table 7 the total systematic uncertainty $\left(\sigma_{\text {sys }}^{\text {total }}\right)$ is given for every class, including the contribution due to Monte Carlo statistics which is shown in the same table with label $M C_{\text {stat }}$.

A consistency test regarding the classified hemispheres is done by looking at the correlation in the classification of the opposite hemispheres of a given $\tau$ pair event. A new matrix of $14 \times 14$ classes is created from the Monte Carlo simulation after weighting every cell by the $\tau$ branching ratios measured from this analysis and taking into account the non- $\tau$ background. 


\begin{tabular}{|c|c|c|c|c|c|c|c|c|c|c|c|c|c|c|}
\hline Mode & 0 & $e$ & $\mu$ & $h$ & $h \pi^{0}$ & $h 2 \pi^{0}$ & $h 3 \pi^{0}$ & $h \geq 4 \pi^{0}$ & $3 h$ & $3 h \pi^{0}$ & $3 h 2 \pi^{0}$ & $3 h 3 \pi^{0}$ & $5 h$ & $5 h \pi^{0}$ \\
\hline 0 & 1.2 & 2.3 & 1.6 & 0.6 & 1.2 & 0.9 & 0.1 & 2.6 & 0.4 & 0.1 & 1.3 & 1.5 & 0.4 & 0.6 \\
\hline$e$ & & 1.1 & 1.3 & 0.8 & 2.0 & 1.1 & 0.1 & 0.6 & 0.3 & 0.3 & 0.3 & 2.2 & 1.8 & 0.9 \\
\hline$\mu$ & & & 1.5 & 0.4 & 1.7 & 1.9 & 1.3 & 0.7 & 2.4 & 0.4 & 0.4 & 1.0 & 0.1 & 1.1 \\
\hline$h$ & & & & 1.4 & 1.1 & 1.4 & 0.3 & 0.2 & 1.4 & 1.0 & 0.7 & 1.1 & 0.1 & 0.1 \\
\hline$h \pi^{0}$ & & & & & 2.1 & 0.7 & 1.0 & 2.5 & 1.1 & 0.2 & 1.7 & 0.7 & 0.6 & 1.5 \\
\hline$h 2 \pi^{0}$ & & & & & & 1.0 & 1.7 & 0.9 & 0.9 & 1.1 & 0.5 & 0.4 & 0.8 & 1.8 \\
\hline$h 3 \pi^{0}$ & & & & & & & 1.1 & 0.1 & 0.4 & 0.1 & 0.1 & 2.0 & 0.6 & 1. \\
\hline$h \geq 4 \pi^{0}$ & & & & & & & & - & 0.2 & 0.6 & 0.5 & 1. & - & - \\
\hline $3 h$ & & & & & & & & & 0.8 & 0.2 & 0.6 & 1.7 & 1.0 & 1.8 \\
\hline $3 h \pi^{0}$ & & & & & & & & & & 0.1 & 1.0 & 0.6 & 1.1 & 0.6 \\
\hline $3 h 2 \pi^{0}$ & & & & & & & & & & & 2. & 0.4 & 0.5 & - \\
\hline $3 h \geq 3 \pi^{0}$ & & & & & & & & & & & & 0.6 & - & - \\
\hline
\end{tabular}

Table 8: Difference between the number of observed $\tau$ pair events in data and that expected from simulation, divided by the statistical error. Bold numbers imply a minus sign. The label " 0 " stands for the small fraction of $\tau$ decays which are not classified, essentially final states with one charged particle (not an electron) with $P<2 \mathrm{GeV} / \mathrm{c}$ and no reconstructed $\pi^{0}$.

The difference between the number of measured $\tau$ pair events (one hemisphere classified as class $i$ and the opposite as $j$ ) on data and that expected from simulation with the measured branching ratios divided by its statistical error is shown in Table 8 for every cell. The overall $\chi^{2}$ of 91.2 for 96 degrees of freedom is satisfactory.

Some of the $\tau$ hemispheres are not classified because they contain one charged particle with a momentum smaller than $2 \mathrm{GeV}$ and no reconstructed $\pi^{0}$ (class 0 in Table 8). The fraction of events with these hemispheres is consistently reproduced by the simulation with a $\chi^{2}$ of 14.8 for 14 degrees of freedom.

\section{Quasi-exclusive and exclusive results}

In this section the results of the global branching ratio analysis are given for the quasi-exclusive and the exclusive classifications. Table 9 shows the results of the quasi-exclusive branching ratio measurements with their statistical errors, and the total estimated systematic error as described in the previous section. These results only refer to given topologies, which are not yet corrected for at this stage for additional kaons. As these results are obtained with a constraint and because of significant mixing between classes with several $\pi^{0}$ 's, the values are 
correlated. In Table 10 the correlation coefficients of the covariance matrix are given for the measured branching ratios.

\begin{tabular}{|l|c|c|c|c|}
\hline $\begin{array}{c}\text { Mode } \\
\text { (before kaon } \\
\text { subtraction })\end{array}$ & Value $\%)$ & $\sigma_{\text {stat }}$ & $\sigma_{\text {sys }}$ & $\sigma_{\text {total }}$ \\
\hline \hline$B\left(\tau \rightarrow h \nu_{\tau}\right)$ & 12.44 & 0.11 & 0.11 & 0.16 \\
\hline$B\left(\tau \rightarrow h \pi^{0} \nu_{\tau}\right)$ & 25.98 & 0.15 & 0.11 & 0.19 \\
\hline$B\left(\tau \rightarrow h 2 \pi^{0} \nu_{\tau}\right)$ & 9.68 & 0.13 & 0.10 & 0.17 \\
\hline$B\left(\tau \rightarrow h 3 \pi^{0} \nu_{\tau}\right)$ & 1.34 & 0.09 & 0.11 & 0.14 \\
\hline$B\left(\tau \rightarrow h \geq 4 \pi^{0} \nu_{\tau}\right)$ & 0.17 & 0.04 & 0.09 & 0.10 \\
\hline$B\left(\tau \rightarrow 3 h \nu_{\tau}\right)$ & 10.02 & 0.10 & 0.09 & 0.13 \\
\hline$B\left(\tau \rightarrow 3 h \pi^{0} \nu_{\tau}\right)$ & 4.45 & 0.09 & 0.07 & 0.12 \\
\hline$B\left(\tau \rightarrow 3 h 2 \pi^{0} \nu_{\tau}\right)$ & 0.54 & 0.07 & 0.07 & 0.10 \\
\hline$B\left(\tau \rightarrow 3 h \geq 3 \pi^{0} \nu_{\tau}\right)$ & 0.11 & 0.04 & 0.05 & 0.06 \\
\hline$B\left(\tau \rightarrow 5 h \nu_{\tau}\right)$ & 0.080 & 0.011 & 0.013 & 0.017 \\
\hline$B\left(\tau \rightarrow 5 h \pi^{0} \nu_{\tau}\right)$ & 0.018 & 0.007 & 0.012 & 0.014 \\
\hline
\end{tabular}

Table 9: Quasi-exclusive hadronic branching ratios before any subtraction for the kaon modes. The first error is statistical, the second systematic, and the last one gives the total uncertainty.

\begin{tabular}{|c|c|c|c|c|c|c|c|c|c|c|c|c|}
\hline Mode & $\mu$ & $h$ & $h \pi^{0}$ & $h 2 \pi^{0}$ & $h 3 \pi^{0}$ & $h \geq 4 \pi^{0}$ & $3 h$ & $3 h \pi^{0}$ & $3 h 2 \pi^{0}$ & $3 h 3 \geq \pi^{0}$ & $5 h$ & $5 h \pi^{0}$ \\
\hline$e$ & -0.190 & -0.151 & -0.261 & -0.094 & -0.029 & -0.006 & -0.121 & -0.073 & -0.009 & -0.009 & -0.006 & -0.001 \\
\hline$\mu$ & 1.000 & -0.148 & -0.246 & -0.086 & -0.029 & -0.005 & -0.116 & -0.069 & -0.009 & -0.009 & -0.006 & -0.001 \\
\hline$h$ & & 1.000 & -0.315 & -0.048 & -0.024 & -0.004 & -0.102 & -0.051 & -0.007 & -0.008 & -0.003 & 0.000 \\
\hline$h \pi^{0}$ & & & 1.000 & -0.413 & 0.011 & -0.007 & -0.160 & -0.115 & -0.012 & -0.016 & -0.009 & -0.001 \\
\hline$h 2 \pi^{0}$ & & & & 1.000 & -0.374 & 0.043 & -0.054 & -0.045 & -0.016 & -0.011 & -0.001 & 0.001 \\
\hline$h 3 \pi^{0}$ & & & & & 1.000 & -0.373 & -0.018 & -0.014 & 0.004 & -0.015 & 0.003 & 0.010 \\
\hline$h \geq 4 \pi^{0}$ & & & & & & 1.000 & -0.002 & -0.007 & -0.012 & -0.022 & 0.008 & -0.003 \\
\hline $3 h$ & & & & & & & 1.000 & -0.235 & 0.001 & -0.004 & -0.032 & +0.001 \\
\hline $3 h \pi^{0}$ & & & & & & & & 1.000 & -0.207 & -0.065 & -0.006 & -0.012 \\
\hline $3 h 2 \pi^{0}$ & & & & & & & & & 1.000 & -0.281 & 0.008 & 0.008 \\
\hline $3 h \geq 3 \pi^{0}$ & & & & & & & & & & 1.000 & 0.006 & 0. \\
\hline $5 h$ & & & & & & & & & & & 1.000 & -0.111 \\
\hline
\end{tabular}

Table 10: Correlation coefficients for the quasi-exclusive analysis. 
An independent global check of the understanding of the $\pi^{0}$ reconstruction is performed by repeating the complete analysis with the exclusive classification. In this way the single photons are not counted as $\pi^{0}$ 's anymore. Instead of rejecting these events a new class is designed for the topologies $\geq 1 h+\geq 0 \pi^{0}+\geq 1 \gamma$, the so-called single photon class. This new class is expected to be populated essentially by "background" from standard decays where at least one photon has been lost. However, a small signal is expected in this last class from the following channels with non- $\pi^{0}$ photons in the final state:

- $B\left(\tau \rightarrow \pi \omega \nu_{\tau}\right) \sim 0.17 \%$ with $\omega \rightarrow \pi^{0} \gamma[17]$

- $B\left(\tau \rightarrow \pi \pi^{0} \eta \nu_{\tau}\right) \sim 0.08 \%$ with $\eta \rightarrow \gamma \gamma, \pi^{+} \pi^{-} \gamma[13]$

- $B\left(\tau \rightarrow \pi \pi^{0} \omega \nu_{\tau}\right) \sim 0.04 \%$ with $\omega \rightarrow \pi^{0} \gamma[12]$.

\begin{tabular}{|c|c|c|c|c|c|c|c|c|c|c|c|c|c|}
\hline Mode & $e$ & $\mu$ & $h$ & $h \pi^{0}$ & $h 2 \pi^{0}$ & $h 3 \pi^{0}$ & $h 4 \pi^{0}$ & $3 h$ & $3 h \pi^{0}$ & $3 h 2 \pi^{0}$ & $3 h 3 \pi^{0}$ & $5 h$ & $5 h \pi^{0}$ \\
\hline 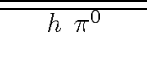 & $\begin{array}{c}0.16 \\
\pm 0.01\end{array}$ & $\begin{array}{c}0.07 \\
\pm 0.01\end{array}$ & $\begin{array}{c}1.24 \\
\pm 0.04\end{array}$ & $\begin{array}{l}\mathbf{5 4 . 5 0} \\
\pm 0.11\end{array}$ & $\begin{array}{c}9.67 \\
\pm 0.11\end{array}$ & $\begin{array}{c}1.87 \\
\pm 0.13\end{array}$ & $\begin{array}{c}0.81 \\
\pm 0.41\end{array}$ & $\begin{array}{c}0.30 \\
\pm 0.02\end{array}$ & $\begin{array}{c}0.80 \\
\pm 0.05\end{array}$ & $\begin{array}{c}0.18 \\
\pm 0.06\end{array}$ & $\begin{array}{c}0.10 \\
\pm 0.10\end{array}$ & $\overline{0 .}$. & $\overline{0 .}$. \\
\hline$h 2 \pi^{0}$ & 0. & 0 . & $\begin{array}{c}0.10 \\
\pm 0.01\end{array}$ & $\begin{array}{c}1.04 \\
\pm 0.02\end{array}$ & $\begin{array}{l}\mathbf{3 0 . 9 7} \\
\pm 0.17\end{array}$ & $\begin{array}{l}14.74 \\
\pm 0.33\end{array}$ & $\begin{array}{c}4.88 \\
\pm 1.09\end{array}$ & $\begin{array}{c}0.03 \\
\pm 0.01\end{array}$ & $\begin{array}{c}0.21 \\
\pm 0.02\end{array}$ & $\begin{array}{c}0.75 \\
\pm 0.12\end{array}$ & $\begin{array}{c}0.38 \\
\pm 0.19\end{array}$ & 0 . & $\begin{array}{c}0.24 \\
\pm 0.23\end{array}$ \\
\hline$h 3 \pi^{0}$ & 0 . & 0 . & $\begin{array}{c}0.01 \\
\pm 0.01\end{array}$ & $\begin{array}{c}0.06 \\
\pm 0.01\end{array}$ & $\begin{array}{c}1.00 \\
\pm 0.04\end{array}$ & $\begin{array}{l}\mathbf{1 7 . 2 0} \\
\pm 0.36\end{array}$ & $\begin{array}{l}32.43 \\
\pm 2.12\end{array}$ & 0 . & $\begin{array}{c}0.04 \\
\pm 0.01\end{array}$ & $\begin{array}{c}0.16 \\
\pm 0.06\end{array}$ & $\begin{array}{c}0.48 \\
\pm 0.21\end{array}$ & 0 . & 0. \\
\hline$h \geq 4 \pi^{0}$ & 0. & 0. & 0. & 0. & $\begin{array}{c}0.03 \\
\pm 0.01\end{array}$ & $\begin{array}{c}0.28 \\
\pm 0.05\end{array}$ & $\begin{array}{c}4.48 \\
\pm 0.92\end{array}$ & 0. & 0. & 0. & $\begin{array}{c}0.19 \\
\pm 0.14\end{array}$ & 0. & 0 . \\
\hline $3 h \pi^{0}$ & 0. & 0. & $\begin{array}{c}0.13 \\
\pm 0.01\end{array}$ & $\begin{array}{c}0.29 \\
\pm 0.01\end{array}$ & $\begin{array}{c}0.17 \\
\pm 0.01\end{array}$ & $\begin{array}{c}0.06 \\
\pm 0.02\end{array}$ & 0. & $\begin{array}{c}2.75 \\
\pm 0.06\end{array}$ & $\begin{array}{r}\mathbf{4 3 . 4 4} \\
\pm 0.26\end{array}$ & $\begin{array}{c}9.81 \\
\pm 0.46\end{array}$ & $\begin{array}{c}3.25 \\
\pm 0.55\end{array}$ & $\begin{array}{c}3.44 \\
\pm 0.76\end{array}$ & $\begin{array}{c}9.92 \\
\pm 1.44\end{array}$ \\
\hline $3 h 2 \pi^{0}$ & 0. & 0 . & $\begin{array}{c}0.04 \\
\pm 0.01\end{array}$ & $\begin{array}{c}0.08 \\
\pm 0.01\end{array}$ & $\begin{array}{c}0.29 \\
\pm 0.02\end{array}$ & $\begin{array}{c}0.28 \\
\pm 0.05\end{array}$ & $\begin{array}{c}0.38 \\
\pm 0.28\end{array}$ & $\begin{array}{c}0.31 \\
\pm 0.02\end{array}$ & $\begin{array}{c}2.78 \\
\pm 0.09\end{array}$ & $\begin{array}{l}\mathbf{2 4 . 5 3} \\
\pm 0.59\end{array}$ & $\begin{array}{c}18.62 \\
\pm 1.22\end{array}$ & $\begin{array}{c}0.19 \\
\pm 0.19\end{array}$ & $\begin{array}{c}3.22 \\
\pm 0.85\end{array}$ \\
\hline $3 h \geq 3 \pi^{0}$ & 0. & 0 . & $\begin{array}{c}0.02 \\
\pm 0.01\end{array}$ & $\begin{array}{c}0.04 \\
\pm 0.01\end{array}$ & $\begin{array}{c}0.11 \\
\pm 0.01\end{array}$ & $\begin{array}{c}0.39 \\
\pm 0.06\end{array}$ & $\begin{array}{c}1.00 \\
\pm 0.45\end{array}$ & $\begin{array}{c}0.06 \\
\pm 0.01\end{array}$ & $\begin{array}{c}0.25 \\
\pm 0.03\end{array}$ & $\begin{array}{c}2.54 \\
\pm 0.21\end{array}$ & $\begin{array}{r}\mathbf{1 5 . 2 0} \\
\pm 1.11\end{array}$ & 0. & $\begin{array}{c}0.47 \\
\pm 0.33\end{array}$ \\
\hline $5 h \pi^{0}$ & 0. & 0 . & 0. & 0 . & 0 . & 0. & 0. & $\begin{array}{c}0.01 \\
\pm 0.01\end{array}$ & $\begin{array}{c}0.01 \\
\pm 0.01\end{array}$ & 0 . & 0. & $\begin{array}{c}0.95 \\
\pm 0.43\end{array}$ & $\begin{array}{l}\mathbf{2 4 . 7 4} \\
\pm 2.14\end{array}$ \\
\hline
\end{tabular}

Table 11: Efficiency matrix for the exclusive classification of hadronic decays. The efficiencies are expressed in percent with their statistical error only. The generated classes are given in the first row, and the reconstructed classes in the first column.

In order to illustrate the exclusive classification, the efficiency matrix is shown in Table 11 for the classes containing at least one $\pi^{0}$ (otherwise there is no change with respect to the previous quasi-exclusive classification). As the exclusive classification is more rigorous with the $\pi^{0}$ identification, less mixing is obtained in the classes with several $\pi^{0}$ 's. The invariant mass distributions for the $h 3 \pi^{0}, h \geq 4 \pi^{0}, 3 h 2 \pi^{0}$ and $3 h \geq 3 \pi^{0}$ are plotted in Figure 23 . An enhancement of the signal over the background is observed with respect to the quasi-exclusive classification.

Table 12 shows the results of the exclusive analysis. For the classes with no $\pi^{0}$ the results are essentially unchanged from the quasi-exclusive analysis. On the other hand, for 

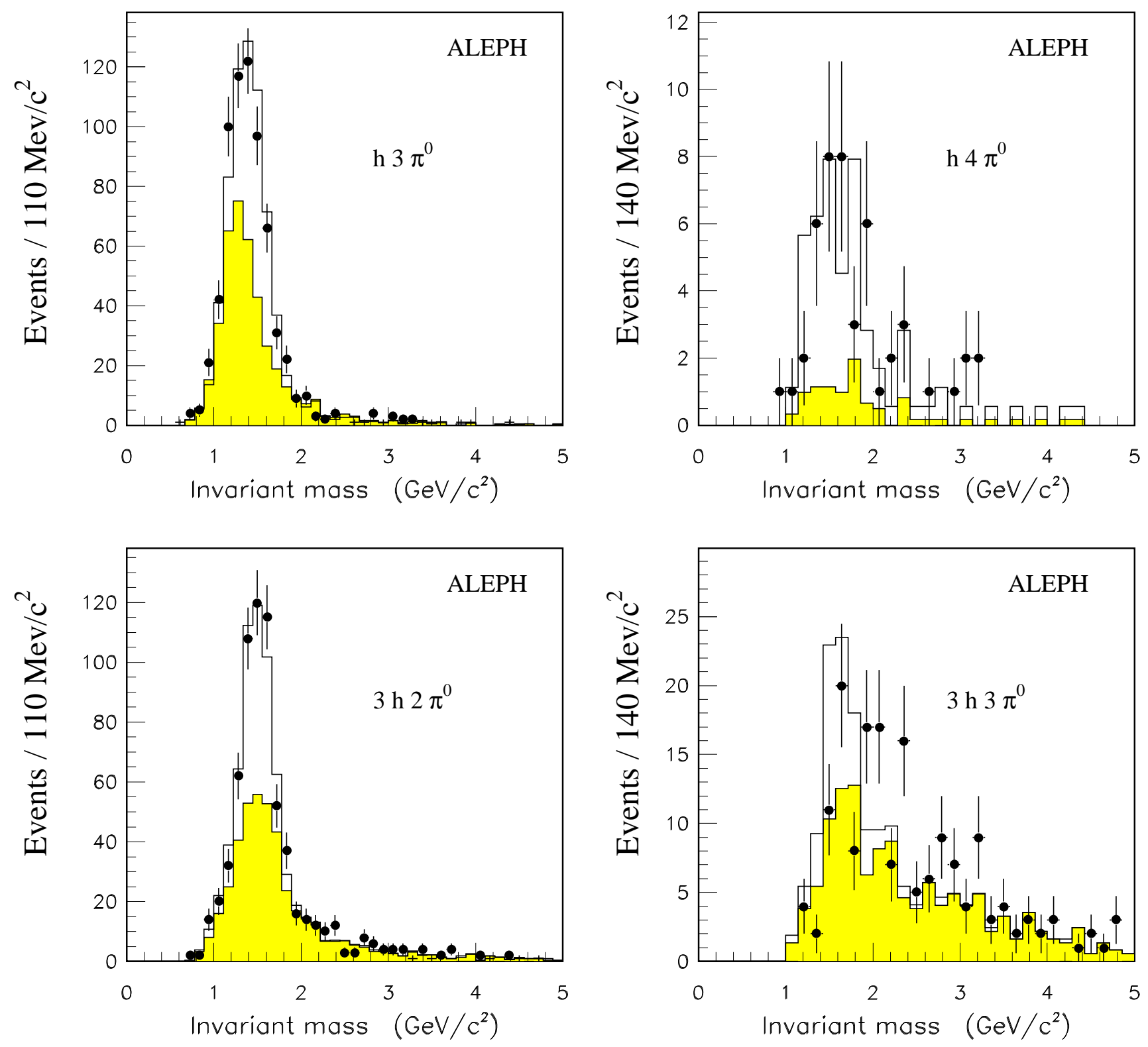

Figure 23: Invariant mass distributions for the $h 3 \pi^{0}, h \geq 4 \pi^{0}, 3 h 2 \pi^{0}$ and $3 h 3 \pi^{0}$ exclusive selected samples. The points with error bars show the observed distributions, the solid histograms represent the simulated distributions and the shaded histograms account for the expected background from other $\tau$ decays with the measured branching ratios. 


\begin{tabular}{|l|c|c|c||c|c|c|}
\hline Mode & Value $(\%)$ & $\sigma_{\text {stat }}$ & $\sigma_{\text {sys }}$ & $\Delta B_{\text {expected }}$ & $\Delta B$ & $\sigma_{\Delta B}$ \\
\hline \hline$B\left(\tau \rightarrow h \nu_{\tau}\right)$ & 12.44 & 0.11 & 0.10 & - & - & - \\
\hline$B\left(\tau \rightarrow h \pi^{0} \nu_{\tau}\right)$ & 25.76 & 0.17 & 0.16 & - & 0.22 & 0.16 \\
\hline$B\left(\tau \rightarrow h 2 \pi^{0} \nu_{\tau}\right)$ & 9.52 & 0.20 & 0.25 & 0.17 & 0.16 & 0.25 \\
\hline$B\left(\tau \rightarrow h 3 \pi^{0} \nu_{\tau}\right)$ & 0.85 & 0.22 & 0.21 & 0.10 & 0.50 & 0.20 \\
\hline$B\left(\tau \rightarrow h \geq 4 \pi^{0} \nu_{\tau}\right)$ & 0.37 & 0.10 & 0.12 & - & -0.21 & 0.11 \\
\hline \hline$B\left(\tau \rightarrow 3 h \nu_{\tau}\right)$ & 10.02 & 0.10 & 0.07 & - & - & - \\
\hline$B\left(\tau \rightarrow 3 h \pi^{0} \nu_{\tau}\right)$ & 4.41 & 0.09 & 0.08 & - & 0.04 & 0.08 \\
\hline$B\left(\tau \rightarrow 3 h 2 \pi^{0} \nu_{\tau}\right)$ & 0.49 & 0.08 & 0.10 & 0.01 & 0.05 & 0.10 \\
\hline$B\left(\tau \rightarrow 3 h 3 \pi^{0} \nu_{\tau}\right)$ & 0.20 & 0.06 & 0.06 & - & -0.10 & 0.06 \\
\hline \hline$B\left(\tau \rightarrow 5 h \nu_{\tau}\right)$ & 0.080 & 0.011 & 0.009 & - & - & - \\
\hline$B\left(\tau \rightarrow 5 h \pi^{0} \nu_{\tau}\right)$ & 0.008 & 0.006 & 0.009 & - & 0.010 & 0.007 \\
\hline \hline$B\left(\tau \rightarrow \geq 1 \mathrm{~h} \geq 0 \pi^{0} \geq 1 \gamma \nu_{\tau}\right)$ & 0.67 & 0.22 & 0.47 & 0.28 & - & - \\
\hline
\end{tabular}

Table 12: Exclusive hadronic branching ratios and differences with respect to the quasiexclusive measurements.

the modes with $\pi^{0}$ s, the differences $\Delta B$ (quasi-exclusive branching ratio minus exclusive branching ratio) are given in Table 12 . The statistical error $\left(\sigma_{\text {stat }}\right)$ and the systematic error $\left(\sigma_{\text {sys }}\right)$ of the exclusive branching ratios are also specified. In order to evaluate the systematic error in the measurement of the exclusive branching ratios the procedure described in Section 9 is repeated with the exclusive classification. The largest differences appear in the systematic uncertainties affecting the $\pi^{0}$ reconstruction as one would expect, since any uncertainty which causes the loss of a photon implies, consequently, the loss of its associated $\pi^{0}$. This is not the case in the quasi-exclusive classification. The systematic uncertainties associated with the exclusive branching ratios, shown in Table 12, are therefore substantially larger than those given in Table 9.

The errors $\sigma_{\Delta B}$ on $\Delta B$ given in Table 12 include the error due to the statistical fluctuation of events not in common and to uncorrelated systematics. The changes in all classes are consistent within the errors and with the expected "hadronic" signal (mainly, in the $h 2 \pi^{0}$ and $h 3 \pi^{0}$ channels ) from decays with photons not coming from $\pi^{0}$ s. However, the overall agreement is only fair with a $\chi^{2}$ of 14.4 for 8 degrees of freedom. Although no clear pattern emerges in Table 12 to explain this fact, it should be reminded that the exclusive branching ratio analysis is more sensitive to systematic uncertainties in the $\pi^{0}$ reconstruction. 
For the $\geq 1 h+\geq 0 \pi^{0}+\geq 1 \gamma$ class (dominated by background from other classes, which is properly subtracted out by the method) a signal of $(0.67 \pm 0.22 \pm 0.47) \%$ is observed, where the first error is statistical and the second systematic. After subtraction of the expected signal, a limit is derived for a new non- $\pi^{0}$ photonic channel (excluding radiative photons which have been eliminated earlier as discussed in Section 6.3), which is found to be:

$$
B_{\text {new }}\left(\tau \rightarrow \geq 1 h+\text { non- } \pi^{0} \gamma^{\prime} s \nu_{\tau}\right)<1.6 \% \text { at } 95 \% \text { CL. }
$$

In deriving (6), the systematic error has been conservatively scaled by a factor of 1.34 to take into account the $\chi^{2}$ of the channel to channel comparison given in Table 12 and quoted above.

In conclusion, from the comparison of the branching ratios obtained with the quasiexclusive and exclusive classifications, no inconsistency is found, and the description of the hadronic modes is complete at the level of precision quoted. Therefore, since the quasiexclusive results are less affected by the systematic effects and are statistically more precise these values are taken as the final results and they are discussed in the next sections.

\section{Update on $\tau$ decays to charged and neutral kaons}

The study of charged and neutral kaon production in $\tau$ decays given in reference [5, 6$]$ was mainly limited by the statistics of the data sample. The same approach is followed when analyzing the 1993 data sample; the reader is referred to the previous publications [5, 6] for more details. A summary of the selected numbers of events, efficiencies and contaminations in the different $\tau$ decays into kaons is given in Table 13. The charged kaon fraction in a given sample is derived by fitting the observed ionization loss distribution to a linear combination of the expected pion and kaon distributions. The difference between the $d E / d x$ measurement and the expectation value for a pion divided by the experimental resolution $\left(x_{\pi}\right)$ is shown in Figure 24 for one-prong hadronic $\tau$ decays for the whole data sample. The fitted distribution is plotted, with the $\pi, e$ and $\mu$ individual contributions and the derived $K$ component. The ratio of the $d E / d x$ probability density for kaons to the sum of the probability densities for kaons and pions defines a kaon estimator $P_{K}$. The distributions of $P_{K}$ are plotted in Figure 25 for the $h K_{L}^{0}$ and $h \pi^{0} K_{L}^{0}$ samples, where the charged kaon signal is evident and in agreement with the simulation.

For completeness, the distributions of the relative excess of energy in the hadronic calorimeter $\left(\delta_{E}\right)$ with respect to the charged particle momentum and the angular displacement of the hadronic energy barycentre $\left(\delta_{\phi}\right)$ with respect to the impact of the charged track are shown in Figure 26 for the $h K_{L}^{0}$ and $h \pi^{0} K_{L}^{0}$ samples. Consistency is observed with the Monte Carlo for both samples. The invariant mass distribution for the $K^{*}$ resonance is plotted in Figure 27 for the $\pi K_{L}^{0}$ sample. Finally, the updated branching ratios for these kaons modes are reported in Table 14. 


\begin{tabular}{|c|c|c|c|}
\hline Mode & Decays & Efficiency (\%) & Background (\%) \\
\hline \hline$\tau \rightarrow K^{-} \geq 0 \pi^{0} K^{0} \nu_{\tau}$ & $1610 \pm 55$ & $63.8 \pm 0.7$ & - \\
\hline$\tau \rightarrow K^{-} \nu_{\tau}$ & $728 \pm 37$ & $55.6 \pm 1.0$ & $14 \pm 4$ \\
\hline$\tau \rightarrow K^{-} \pi^{0} \nu_{\tau}$ & $395 \pm 27$ & $44.0 \pm 1.4$ & $10 \pm 3$ \\
\hline$\tau \rightarrow K^{-} 2 \pi^{0} \nu_{\tau}$ & $59 \pm 11$ & $30.3 \pm 1.8$ & $37 \pm 13$ \\
\hline$\tau \rightarrow \pi^{-} \overline{K^{0}} \nu_{\tau}$ & $98 \pm 10$ & $6.3 \pm 0.2$ & $19 \pm 5$ \\
\hline$\tau \rightarrow K^{-} K^{0} \nu_{\tau}$ & $13 \pm 4$ & $3.0 \pm 0.2$ & $8 \pm 8$ \\
\hline$\tau \rightarrow \pi^{-} \pi^{0} \overline{K^{0}} \nu_{\tau}$ & $23 \pm 5$ & $2.9 \pm 0.4$ & $35 \pm 9$ \\
\hline$\tau \rightarrow K^{-} \pi^{0} K^{0} \nu_{\tau}$ & $5 \pm 2$ & $2.9 \pm 0.8$ & $20 \pm 20$ \\
\hline
\end{tabular}

Table 13: Summary of statistics, efficiencies and backgrounds for the $\tau$ decay modes containing kaons.

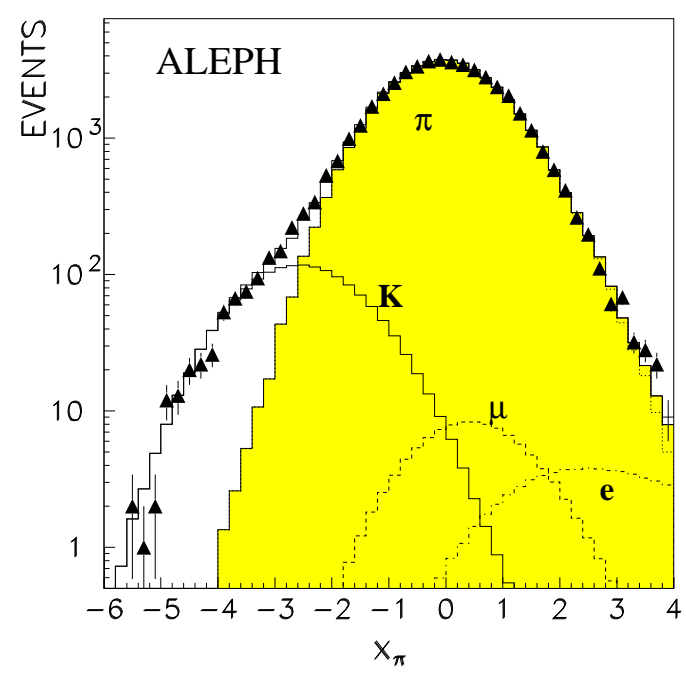

Figure 24: Fitted $x_{\pi}$ distribution in one-prong hadronic $\tau$ decays. The points with error bars correspond to data, the solid lines show the $\pi, e$ and $\mu$ contributions and the fitted $K$ component. 

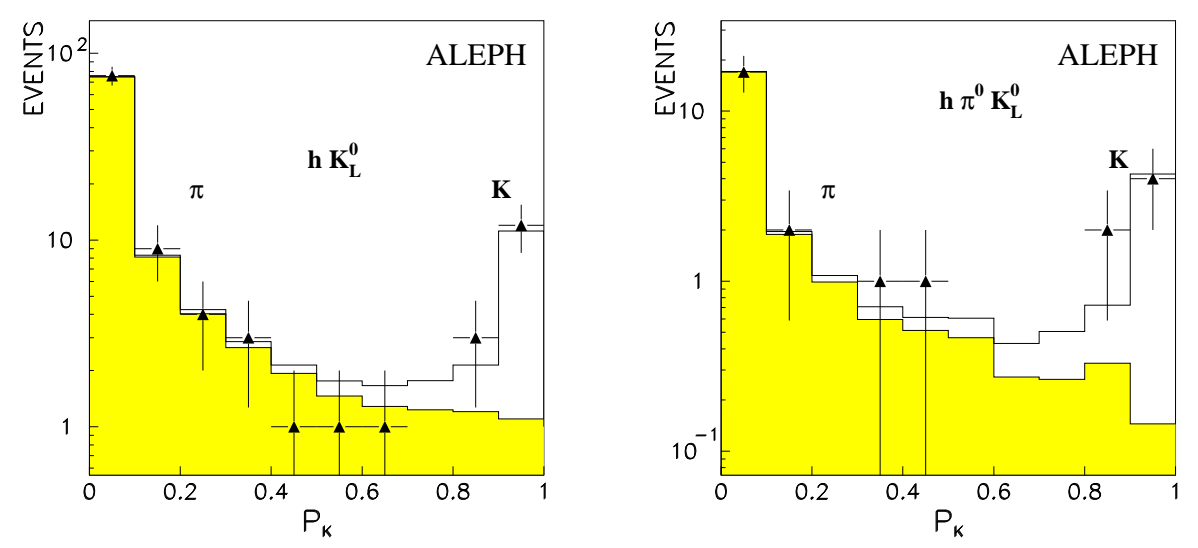

Figure 25: The distributions of the charged kaon $d E / d x$ estimator for the $h K_{L}^{0}$ and $h \pi^{0} K_{L}^{0}$ selected samples. The points with error bars show the observed distribution, and the solid histogram the simulation. The shaded histograms show the expected $\pi$ contribution.

\begin{tabular}{|l|c|c|c|c|}
\hline Mode & Value $(\%)$ & $\sigma_{\text {stat }}$ & $\sigma_{\text {sys }}$ & $\sigma_{\text {total }}$ \\
\hline \hline$B\left(\tau \rightarrow K^{-} \geq 0 \pi^{0} K^{0} \nu_{\tau}\right)$ & 1.70 & 0.05 & 0.06 & 0.08 \\
\hline$B\left(\tau \rightarrow K^{-} \nu_{\tau}\right)$ & 0.72 & 0.04 & 0.04 & 0.06 \\
\hline$B\left(\tau \rightarrow K^{-} \pi^{0} \nu_{\tau}\right)$ & 0.52 & 0.04 & 0.05 & 0.06 \\
\hline$B\left(\tau \rightarrow K^{-} 2 \pi^{0} \nu_{\tau}\right)$ & 0.08 & 0.02 & 0.02 & 0.03 \\
\hline$B\left(\tau \rightarrow \pi^{-} \overline{K^{0}} \nu_{\tau}\right)$ & 0.79 & 0.10 & 0.09 & 0.13 \\
\hline$B\left(\tau \rightarrow K^{-} K^{0} \nu_{\tau}\right)$ & 0.26 & 0.09 & 0.02 & 0.09 \\
\hline$B\left(\tau \rightarrow \pi^{-} \pi^{0} \overline{K^{0}} \nu_{\tau}\right)$ & 0.32 & 0.11 & 0.05 & 0.12 \\
\hline$B\left(\tau \rightarrow K^{-} \pi^{0} K^{0} \nu_{\tau}\right)$ & 0.10 & 0.05 & 0.03 & 0.06 \\
\hline$B\left(\tau \rightarrow K^{*} \nu_{\tau}\right)$ & 1.39 & 0.09 & 0.10 & 0.13 \\
\hline
\end{tabular}

Table 14: Branching ratios for modes with kaons. The statistical and systematic errors are also shown. 

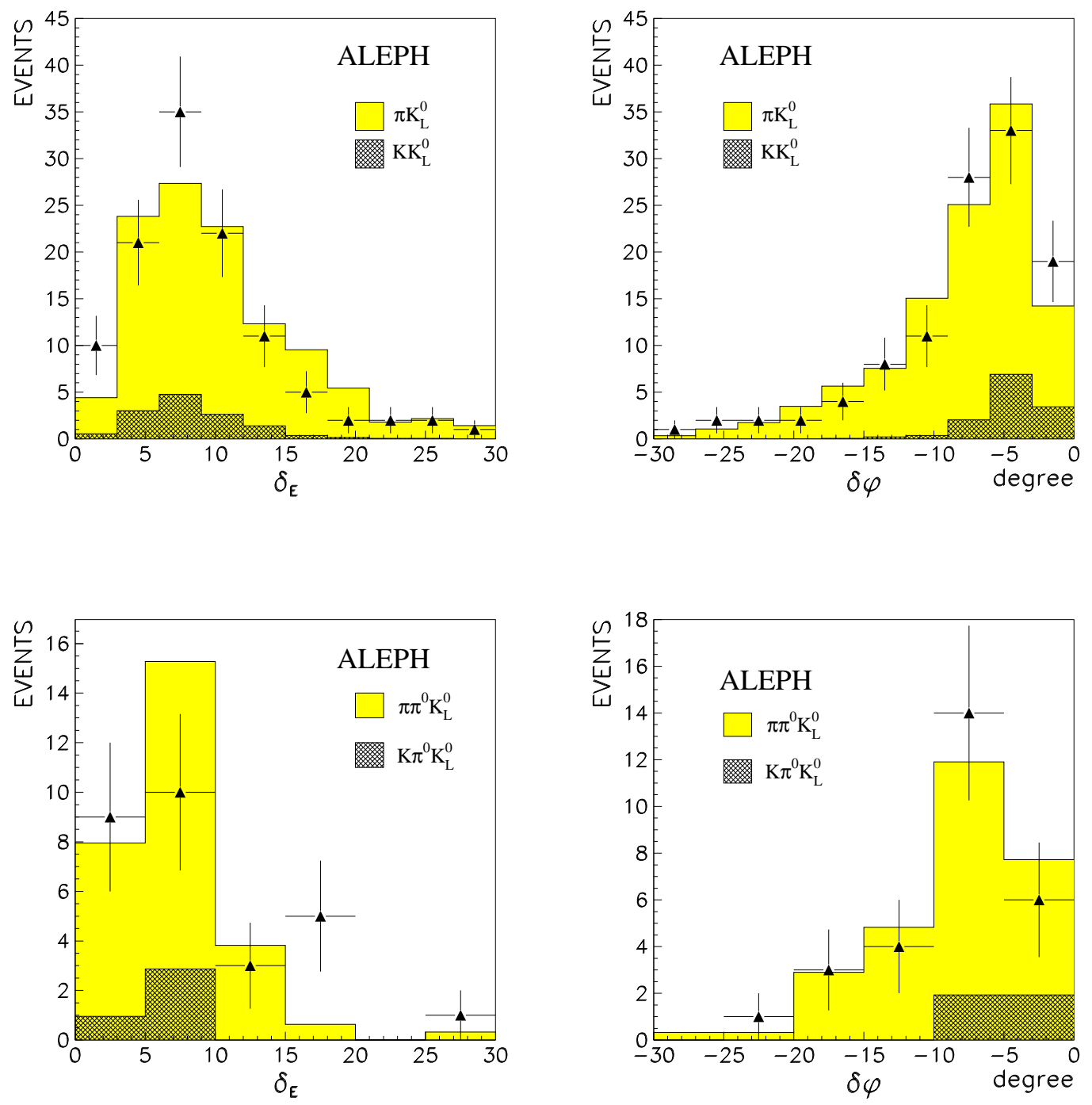

Figure 26: In the upper plots the distributions of $\delta_{E}$ and $\delta_{\phi}$ are shown for the $\pi K_{L}^{0}$ and $K K_{L}^{0}$ samples. The points with error bars show the data for the whole $h K_{L}^{0}$ sample, and the shaded and hatched histograms correspond to the Monte Carlo simulation for the $\pi K_{L}^{0}$ and $K K_{L}^{0}$ samples. The lower plots follow the same specifications as above, but they correspond to the $\pi \pi^{0} K_{L}^{0}$ and $K \pi^{0} K_{L}^{0}$ samples. 

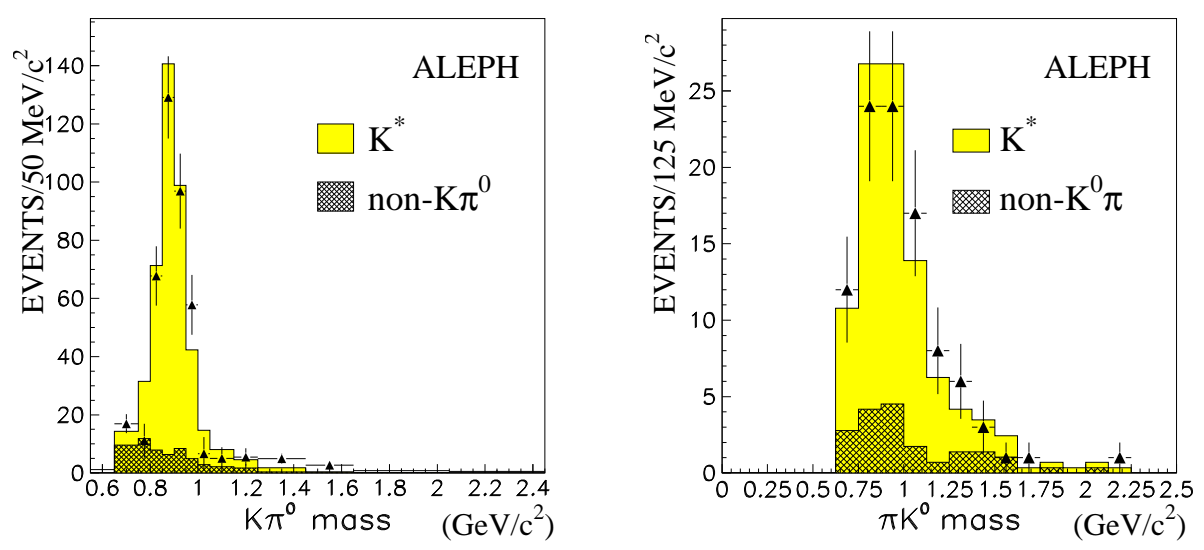

Figure 27: Invariant mass distributions for the $K \pi^{0}$ (left) and $\pi K_{L}^{0}$ (right) systems. The points with error bars show the measured invariant mass distribution, and the shaded histograms stand for the simulation. The hatched histograms correspond to the background in the $K \pi^{0}$ and $\pi K_{L}^{0}$ decays.

\section{Corrected branching ratio results}

The results of the quasi-exclusive classification after subtraction of kaon modes and $\omega, \eta$ decays into non- $\pi^{0}$ photons are given in Table 15 . When subtracting such modes, their efficiencies are taken into account and the final error includes the statistical and systematic components of this correction. These results are consistent with the published ALEPH values with 1989 and 1990 data $[3]$.

The values used to perform such subtractions are described in the following:

- For the $\tau$ decay modes into $K^{-}, K^{-} K^{0}, K^{*^{-}}, K^{-} 2 \pi^{0}, \pi^{-} \pi^{0} \overline{K^{0}}$ and $K^{-} \pi^{0} \overline{K^{0}}$, the measured values given in Table 14 are taken.

- For the $\tau$ decay mode into $\pi \omega$, the omega fraction, $f_{\omega}=(38.3 \pm 2.9) \%$ [17], is also used together with the $3 h \pi^{0}$ decay rate measured in the global analysis.

- For the $\tau$ decay mode into $\pi \pi^{0} \omega, 80 \%$ of the branching ratio for $3 h 2 \pi^{0}$ is used since this channel is observed to be dominated by the $\omega$ resonance [12].

- For the $\tau$ branching ratio into $\pi K^{0} \overline{K^{0}}$ the value $0.3 \%$ is taken with $\pm 50 \%$ uncertainty and the assumption that the final states $\pi K_{L}^{0} K_{L}^{0}, \pi K_{S}^{0} K_{S}^{0}$ and $\pi K_{S}^{0} K_{L}^{0}$ are in the ratios $1: 1: 2$. 
- No attempt is made to subtract the decay modes $K^{-} \pi^{+} \pi^{-}$and $K^{-} K^{+} \pi^{-}$, in the $3 h$ class at this time.

\begin{tabular}{|l|c|c|c|c|}
\hline Mode & Value $(\%)$ & $\sigma_{\text {stat }}$ & $\sigma_{\text {sys }}$ & $\sigma_{\text {total }}$ \\
\hline \hline$B\left(\tau \rightarrow h \nu_{\tau}\right)$ & 11.78 & 0.11 & 0.13 & 0.17 \\
$B\left(\tau \rightarrow \pi \nu_{\tau}\right)$ & 11.06 & 0.11 & 0.14 & 0.18 \\
\hline$B\left(\tau \rightarrow h \pi^{0} \nu_{\tau}\right)$ & 25.76 & 0.15 & 0.13 & 0.20 \\
$B\left(\tau \rightarrow \pi \pi^{0} \nu_{\tau}\right)$ & 25.30 & 0.15 & 0.13 & 0.20 \\
\hline$B\left(\tau \rightarrow h 2 \pi^{0} \nu_{\tau}\right)$ & 9.29 & 0.13 & 0.11 & 0.17 \\
$B\left(\tau \rightarrow \pi 2 \pi^{0} \nu_{\tau}\right)$ & 9.21 & 0.13 & 0.11 & 0.17 \\
\hline$B\left(\tau \rightarrow h 3 \pi^{0} \nu_{\tau}\right)$ & 1.17 & 0.09 & 0.11 & 0.14 \\
\hline$B\left(\tau \rightarrow h \geq 4 \pi^{0} \nu_{\tau}\right)$ & 0.16 & 0.04 & 0.09 & 0.10 \\
\hline \hline$B\left(\tau \rightarrow 3 h \nu_{\tau}\right)$ & 9.50 & 0.10 & 0.11 & 0.15 \\
\hline$B\left(\tau \rightarrow 3 h \pi^{0} \nu_{\tau}\right)$ & 4.30 & 0.09 & 0.09 & 0.12 \\
\hline$B\left(\tau \rightarrow 3 h 2 \pi^{0} \nu_{\tau}\right)$ & 0.50 & 0.07 & 0.07 & 0.10 \\
\hline$B\left(\tau \rightarrow 3 h \geq 3 \pi^{0} \nu_{\tau}\right)$ & 0.11 & 0.04 & 0.05 & 0.06 \\
\hline \hline$B\left(\tau \rightarrow 5 h \nu_{\tau}\right)$ & 0.080 & 0.011 & 0.013 & 0.017 \\
\hline$B\left(\tau \rightarrow 5 h \pi^{0} \nu_{\tau}\right)$ & 0.018 & 0.007 & 0.012 & 0.014 \\
\hline
\end{tabular}

Table 15: Quasi-exclusive hadronic branching ratios after subtraction of the contributions from charged and neutral kaons, and non- $\pi^{0}$ photonic $\omega, \eta$ decays.

Although the leptonic branching ratios are also obtained in the global analysis, they are not discussed in this paper. They are in good agreement with the results of the dedicated analysis described in reference [4]: from the quasi-exclusive classification the corresponding branching fractions for the $\tau \rightarrow e \overline{\nu_{e}} \nu_{\tau}$ and $\tau \rightarrow \mu \overline{\nu_{\mu}} \nu_{\tau}$ decays are $(17.81 \pm 0.11) \%$ and $(17.34 \pm 0.11) \%$, where only the statistical error is given. The corresponding values are $(17.79 \pm 0.12) \%$ and $(17.31 \pm 0.11) \%$ for the dedicated leptonic analysis. The differences are compatible within an expected uncertainty of $0.05 \%$ from statistics and systematics not in common.

\section{Discussion of the results}

In this section the results presented in this paper are analyzed, discussed and compared to theoretical estimates. 


\section{$\tau \rightarrow h \nu_{\tau}$}

The $\tau \rightarrow h \nu_{\tau}$ branching ratio is shown in Figure 28 before any kaon subtraction and compared to the existing measurements. After $K^{0}$ removal, excellent agreement is found between the decay rate of $\tau \rightarrow h \nu_{\tau}(11.78 \pm 0.17) \%$ and the Standard Model predictions equal to $(11.75 \pm 0.06) \%$ and $(11.80 \pm 0.13) \%$ according to references $[24,25]$, respectively, where the ALEPH $\tau$ lifetime measurement of $(293.7 \pm 2.7 \pm 1.6) \mathrm{fs}[26]$ is used. It is possible to express this comparison for the $\tau \rightarrow \pi \nu_{\tau}$ decay as a test of $\mu-\tau$ universality in the $\mathrm{V}-\mathrm{A}$ charged weak current. Using the measured $\tau$ and $\pi$ lifetimes and the branching ratio of the decay $\pi \rightarrow \mu \nu$, together with the radiative corrections relevant to both processes [24, 26, 27], the obtained value is

$$
\frac{g_{\tau}}{g_{\mu}}=1.0015 \pm 0.0098
$$

A similar test is performed with $\tau \rightarrow K \nu_{\tau}$ and $K \rightarrow \mu \nu$ decays, yielding

$$
\frac{g_{\tau}}{g_{\mu}}=0.997 \pm 0.042
$$

Combining both results $(1.0013 \pm 0.0095)$, the $\mu-\tau$ universality hypothesis holds at the $1 \%$ level in these channels.

Moreover, the ratio of the decay rates $\tau \rightarrow K \nu_{\tau}$ and $\tau \rightarrow \pi \nu_{\tau}$

$$
\frac{B\left(\tau \rightarrow K \nu_{\tau}\right)}{B\left(\tau \rightarrow \pi \nu_{\tau}\right)}=0.0651 \pm 0.0055,
$$

measures the ratio of the $f_{\pi}$ and $f_{K}$ decay constants according to the following equation:

$$
\frac{f_{\pi}}{f_{K}}=\tan \theta_{c} \sqrt{\frac{B\left(\tau \rightarrow \pi \nu_{\tau}\right)}{B\left(\tau \rightarrow K \nu_{\tau}\right)}}\left(\frac{m_{\tau}^{2}-m_{K}^{2}}{m_{\tau}^{2}-m_{\pi}^{2}}\right),
$$

once the kinematic factors and the Cabibbo angle $\theta_{c}$ are considered. The ratio of the $\pi$ and $K$ decay constants is found to be:

$$
\frac{f_{\pi}}{f_{K}}=0.825 \pm 0.035
$$

$$
\tau \rightarrow h \pi^{0} \nu_{\tau}
$$

The most precise measurements for the $\tau \rightarrow h \pi^{0} \nu_{\tau}$ branching ratio are drawn in Figure 28 including the kaon contributions as stated in Table 3. Although the earlier measurements favour a lower $h \pi^{0}$ branching ratio, the newer measurements [28, 29, 30], and in particular the present determination prefer a higher value. The hypothesis of the conserved vector current (CVC) allows to derive a value for the ratio of $\tau \rightarrow \pi \pi^{0} \nu_{\tau}$ and $\tau \rightarrow e \overline{\nu_{e}} \nu_{\tau}$ 
using $e^{+} e^{-}$data through an isospin rotation. Isospin invariance of the quark currents coupled to gauge bosons is imbedded in the Standard Model which naturally incorporates the CVC property. From the measured electron branching ratio of $(17.79 \pm 0.12) \%$ [4], the predicted $\tau \rightarrow \pi \pi^{0} \nu_{\tau}$ branching ratio is $(24.6 \pm 0.7) \%$ [31], which is in good agreement with the value of $(25.30 \pm 0.20) \%$ obtained from this analysis within the larger uncertainties arising from the $e^{+} e^{-}$data.

Assuming vector meson dominance, the $K^{*}$ Cabibbo-suppressed decay can be compared to the $\pi \pi^{0}$ decay to extract the ratio of the $\rho$ and $K^{*}$ decay constants according to

$$
\frac{f_{\rho}}{f_{K^{*}}}=\tan \theta_{c} \sqrt{\frac{B\left(\tau \rightarrow \rho \nu_{\tau}\right)}{B\left(\tau \rightarrow K^{*} \nu_{\tau}\right)}}\left(\frac{m_{\tau}^{2}-m_{K^{*}}^{2}}{m_{\tau}^{2}-m_{\rho}^{2}}\right) \sqrt{\frac{m_{\tau}^{2}+2 m_{K^{*}}^{2}}{m_{\tau}^{2}+2 m_{\rho}^{2}}}
$$

which yields

$$
\frac{f_{\rho}}{f_{K^{*}}}=0.931 \pm 0.044
$$

The normalization of the decay constants in the vector current is chosen to have the same units as in the pseudoscalar case. This ratio shows a similar magnitude with the above $f_{\pi} / f_{K}$, and it is in fair agreement with the value of one which can be inferred from the naive application of the Das-Mathur-Okubo (DMO) sum rule in the single-resonance and narrow width assumptions $[32,33]$. In other words, the measured ratio

$$
\frac{B\left(\tau \rightarrow K^{*} \nu_{\tau}\right)}{B\left(\tau \rightarrow \rho \nu_{\tau}\right)}=0.0549 \pm 0.0052
$$

is consistent with the DMO prediction of 0.0476 and is similar in magnitude to the ratio of the $\tau \rightarrow K \nu_{\tau}$ and $\tau \rightarrow \pi \nu_{\tau}$ decay rates.

\section{$\tau \rightarrow h 2 \pi^{0} \nu_{\tau}$ and $\tau \rightarrow 3 h \nu_{\tau}$}

The available measurements for the $\tau \rightarrow h 2 \pi^{0} \nu_{\tau}$ branching ratio are plotted in Figure 28, after subtraction of the $K_{S}^{0}$ component. The ALEPH measurement $(9.29 \pm 0.17) \%$ is consistent with the PDG $94(9.0 \pm 0.4) \%[27]$ value.

Figure 29 shows the branching fractions for $\tau \rightarrow 3 h \nu_{\tau}$, with the $K_{S}^{0}$ component subtracted. Previously published measurements lead to a world average of $(8.0 \pm 0.4) \%$ [27] with measurements spreading over a considerable range. New measurements of this decay rate obtained from $\tau^{\prime}$ 's produced in $Z^{0}$ decays, presented here and in reference [34], are inconsistent with this average. Possible sources of systematic discrepancies have already been discussed [35].

The $3 h$ and $h 2 \pi^{0}$ channels are observed to be dominated by the $a_{1} \rightarrow \rho \pi$ resonance. Therefore, isospin invariance predicts equal decay rates for $3 \pi$ and $\pi 2 \pi^{0}$, ignoring small phase-space corrections. Subtracting the contributions of the decays $\tau \rightarrow K^{-} \pi^{+} \pi^{-} \nu_{\tau}$ and 
$\tau \rightarrow K^{-} K^{+} \pi^{-} \nu_{\tau}[14,15]$ from the measured $3 h$ branching fraction, the value $(8.94 \pm 0.20) \%$ is derived for the $\tau^{-} \rightarrow \pi^{-} \pi^{+} \pi^{-} \nu_{\tau}$ decay. This value is in agreement with the $\tau \rightarrow \pi 2 \pi^{0} \nu_{\tau}$ decay rate of $(9.21 \pm 0.17) \%$. No direct theoretical estimate can be made for the $\tau \rightarrow 3 \pi \nu_{\tau}$ decay rate. However, a consistency argument can be developed through the $\mathrm{V}-\mathrm{A}$ structure of the hadronic current: the axial and vector hadronic widths should be approximately equal. Small non-perturbative QCD corrections are applied [36, 37] and the $\pi$ and $5 \pi$ decay widths (given in this section) are taken into account to derive an estimate of $(18.06 \pm 0.86) \%$ for the total $3 \pi$ width $\left(\pi^{-} \pi^{+} \pi^{-}\right.$and $\left.\pi^{-} 2 \pi^{0}\right)$. This value compares well to the above result of $(18.15 \pm 0.26) \%$.

$$
\tau \rightarrow h 3 \pi^{0} \nu_{\tau}, \tau \rightarrow 3 h \pi^{0} \nu_{\tau}, \tau \rightarrow 5 h \pi^{0} \nu_{\tau} \text { and } \tau \rightarrow 3 h 3 \pi^{0} \nu_{\tau}
$$

For the $h 3 \pi^{0}$ decay mode, the ALEPH measurement shows good agreement with the published CLEO result [38], as drawn in Figure 28. In addition, the CVC prediction for the $\tau \rightarrow \pi 3 \pi^{0} \nu_{\tau}$ decay, $(1.07 \pm 0.05) \%$ [31], is consistent with the ALEPH result of $(1.17 \pm 0.14) \%$.

Significant progress is achieved in the three-prong $\tau$ decays where exclusive decays containing up to three $\pi^{0}$ s are reported in this paper. There are two CVC predictions for the $\tau \rightarrow 3 \pi \pi^{0} \nu_{\tau}$ decay, $(4.2 \pm 0.3) \%[31]$ and $(4.8 \pm 0.7) \%$ [36], which are in agreement with the measured value $(4.30 \pm 0.12) \%$.

The $5 h \pi^{0}$ branching fraction is in agreement with the recently published CLEO results [39], as plotted in Figure 29. From the CVC prediction for $\tau \rightarrow 6 \pi \nu_{\tau}$ and isospin symmetry, a lower limit for the $\tau \rightarrow 5 \pi \pi^{0} \nu_{\tau}$ decay equal to $(0.027 \pm 0.005) \%[31]$ is inferred, which is close to the ALEPH measurement $(0.018 \pm 0.014) \%$. For the decay $\tau \rightarrow 3 h 3 \pi^{0}$ a branching ratio value of $(0.11 \pm 0.06) \%$ is obtained. This value is larger than the estimates from CVC or $5 h \pi^{0}$ using isospin symmetry, which are in the range $(0.02-0.08) \%$, but it is consistent within the errors. In fact, the sum of the $5 h \pi^{0}$ and the $3 h 3 \pi^{0}$ decays adds up to $(0.128 \pm 0.062) \%$ in agreement with the CVC prediction of $(0.13 \pm 0.02) \%$ for the six pion final state.

$$
\tau \rightarrow h 4 \pi^{0} \nu_{\tau}, \tau \rightarrow 3 h 2 \pi^{0} \nu_{\tau} \text { and } \tau \rightarrow 5 h \nu_{\tau}
$$

The $\tau \rightarrow h 4 \pi^{0} \nu_{\tau}, \tau \rightarrow 3 h 2 \pi^{0} \nu_{\tau}$ and $\tau \rightarrow 5 h \nu_{\tau}$ branching ratios show a agreement with the CLEO measurements $[12,38,39]$ as plotted in Figures 28 and 29. In fact, a higher decay rate for $\tau \rightarrow 3 h 2 \pi^{0} \nu_{\tau}$, when compared to its isospin partner $\tau \rightarrow 5 h \nu_{\tau}$, is found. From the measurements given in Table 15, the branching ratio for the five pion final state is found to be $(0.65 \pm 0.14) \%$, once the $\tau \rightarrow \eta \pi \pi^{0} \nu_{\tau}$ contribution is subtracted out. This result is in rough agreement with the $\sim 1 \%$ prediction using the partially conserved axial current hypothesis [40].

Finally, Figure 30 shows exclusive $\tau$ decay branching ratios involving kaons in the final state. The ALEPH measurements are in agreement with the CLEO and L3 results [41, 42]. Avoiding the difficulty to select the isovector part from the isoscalar component in $e^{+} e^{-}$data, 
several crude theoretical estimates exist for the $\tau \rightarrow K K^{0} \nu_{\tau}$ decay neglecting possible SU(3) breaking effects. These estimates give $(0.13 \pm 0.03) \%$ [31] and $(0.16 \pm 0.02) \%$ [36], slightly lower than the present ALEPH result of $(0.26 \pm 0.09) \%$.

\section{$R_{\tau, V}$ and $R_{\tau, A}$}

From the complete analysis of the $\tau$ hadronic branching ratios presented in this paper, it is possible to determine the vector and axial vector contributions to the total $\tau$ hadronic width, usually expressed in terms of their ratios to the leptonic width, called $R_{\tau, V}$ and $R_{\tau, A}$ respectively. The Cabibbo-suppressed contribution is excluded here. As discussed in reference [43] the $R_{\tau, V}$ and $R_{\tau, A}$ observables are of great interest since in their difference the non-perturbative contributions are enhanced, allowing a confrontation with the existing phenomenological models.

Modes involving a $K \bar{K}$ pair in the final state may contribute to both vector and axial vector channels, although one can expect a dominant vector contribution [43]. In the following, they are equally divided between the vector and axial vector parts with an uncertainty of $100 \%$. For the $\tau \rightarrow \eta \pi \pi^{0} \nu_{\tau}$ decay the CLEO result is used [13]. For the $\tau \rightarrow \pi K^{0} \overline{K^{0}} \nu_{\tau}$ decay rate the value $(0.3 \pm 0.15) \%$ is assumed. For $\tau \rightarrow K \bar{K} \pi \nu_{\tau}$ and $\tau \rightarrow K \pi \pi \nu_{\tau}$ fractions the values from references $[14,15]$ are used.

More explicitly, the different branching ratios contributing to the vector and axial $\tau$ decay widths are given in Table 16. Attention is paid to G-parity violating decays of $\eta$ 's and $\omega$ 's ( $\omega \rightarrow \pi^{0} \gamma$ and $\omega \rightarrow \pi^{-} \pi^{+}$with branching ratios taken from reference [27]).

Using the ALEPH $\tau \rightarrow e \overline{\nu_{e}} \nu_{\tau}$ and $\tau \rightarrow \mu \overline{\nu_{\mu}} \nu_{\tau}$ branching ratios [4], assuming $\mu-$ $e$ universality in the weak charged current and subtracting the Cabibbo-suppressed modes (Table 16), one obtains

$$
R_{\tau, V}+R_{\tau, A}=3.485 \pm 0.029
$$

From the present analysis, the following values are obtained using the results of Tables 15 and 14, the ALEPH leptonic branching ratio as above, and the complete covariance matrix

$$
\begin{aligned}
R_{\tau, V} & =1.788 \pm 0.019 \pm 0.016 \\
R_{\tau, A} & =1.694 \pm 0.022 \pm 0.016 \\
R_{\tau, V}-R_{\tau, A} & =0.094 \pm 0.031 \pm 0.032
\end{aligned}
$$

where the first error contains the statistical and systematic contributions and the second accounts for the uncertainty in the vector axial components of the decay rates containing a

$K \bar{K}$ pair. The sum of the values for $R_{\tau, V}$ and $R_{\tau, A}$ does not exactly add up to (9) because of the slight difference in leptonic branching ratios discussed in Section 10.

These results are in agreement with the phenomenological estimate of reference [43]. They provide additional support for the validity of a perturbative QCD description of hadronic $\tau$ 


\begin{tabular}{|l|c|c|}
\hline \multicolumn{1}{|c|}{ Mode } & Value $(\%)$ & Decay type \\
\hline \hline$B\left(\tau \rightarrow \pi^{-} \nu_{\tau}\right)$ & $11.06 \pm 0.18$ & $\mathrm{~A}$ \\
\hline$B\left(\tau \rightarrow \pi^{-} \pi^{0} \nu_{\tau}\right)$ & $25.30 \pm 0.20$ & $\mathrm{~V}$ \\
\hline$B\left(\tau \rightarrow \pi^{-} 2 \pi^{0} \nu_{\tau}\right)$ & $9.21 \pm 0.17$ & $\mathrm{~A}$ \\
\hline$B\left(\tau \rightarrow h^{-} 3 \pi^{0} \nu_{\tau}\right)$ & $0.11 \pm 0.10$ & $\mathrm{~V}$ \\
\hline$B\left(\tau \rightarrow h^{-} 4 \pi^{0} \nu_{\tau}\right)$ & $8.90 \pm 0.20$ & $\mathrm{~A}$ \\
\hline$B\left(\tau \rightarrow \pi^{-} \pi^{+} \pi^{-} \nu_{\tau}\right)$ & $2.66 \pm 0.07$ & $\mathrm{~V}$ \\
\hline$B\left(\tau \rightarrow 2 h^{-} h^{+} \pi^{0} \nu_{\tau}\right)$ & $1.84 \pm 0.05 \pm 0.14$ & $\mathrm{~V}$ \\
\hline$B\left(\tau \rightarrow \pi^{-} \omega \nu_{\tau}\right)$ & $0.09 \pm 0.02$ & $\mathrm{~A}$ \\
\hline$B\left(\tau \rightarrow 2 h^{-} h^{+} 2 \pi^{0} \nu_{\tau}\right)$ & $0.41 \pm 0.08 \pm 0.06$ & $\mathrm{~A}$ \\
\hline$B\left(\tau \rightarrow \pi^{-} \pi^{0} \omega \nu_{\tau}\right)$ & $0.11 \pm 0.06$ & $\mathrm{~V}$ \\
\hline$B\left(\tau \rightarrow 2 h^{-} h^{+} 3 \pi^{0} \nu_{\tau}\right)$ & $0.080 \pm 0.017$ & $\mathrm{~A}$ \\
\hline$B\left(\tau \rightarrow 3 h^{-} 2 h^{+} \nu_{\tau}\right)$ & $0.018 \pm 0.014$ & $\mathrm{~V}$ \\
\hline$B\left(\tau \rightarrow 3 h^{-} 2 h^{+} \pi^{0} \nu_{\tau}\right)$ & $0.72 \pm 0.06$ & $\mathrm{~S}$ \\
\hline$B\left(\tau \rightarrow K^{-} \nu_{\tau}\right)$ & $1.39 \pm 0.13$ & $\mathrm{~S}$ \\
\hline$B\left(\tau \rightarrow K^{*} \nu_{\tau}\right)$ & $0.08 \pm 0.03$ & $\mathrm{~S}$ \\
\hline$B\left(\tau \rightarrow K^{-} 2 \pi^{0} \nu_{\tau}\right)$ & $0.26 \pm 0.09$ & $\mathrm{~V}$ \\
\hline$B\left(\tau \rightarrow K^{-} K^{0} \nu_{\tau}\right)$ & $0.32 \pm 0.12$ & $\mathrm{~S}$ \\
\hline$B\left(\tau \rightarrow \pi^{-} \pi^{0} \overline{K^{0}} \nu_{\tau}\right)$ & $0.10 \pm 0.06$ & $50 \% \mathrm{~V}, \mathrm{~A}$ \\
\hline$B\left(\tau \rightarrow K^{-} \pi^{0} K^{0} \nu_{\tau}\right)$ & $0.39 \pm 0.12$ & $\mathrm{~S}$ \\
\hline \hline$B\left(\tau \rightarrow K^{-} \pi^{+} \pi^{-} \nu_{\tau}\right)[14,15]$ & $0.17 \pm 0.07$ & $50 \% \mathrm{~V}, \mathrm{~A}$ \\
\hline$B\left(\tau \rightarrow K^{-} K^{+} \pi^{-} \nu_{\tau}\right)[14,15]$ & $0.30 \pm 0.15$ & $50 \% \mathrm{~V}, \mathrm{~A}$ \\
\hline$B\left(\tau \rightarrow \pi^{-} K^{0} \overline{K^{0}} \nu_{\tau}\right)[16]$ & $0.17 \pm 0.03$ & $\mathrm{~V}$ \\
\hline$B\left(\tau \rightarrow \pi^{-} \pi^{0} \eta \nu_{\tau}\right)[13]$ & & \\
\hline
\end{tabular}

Table 16: The measured branching ratios and values used, separated into vector (V), axial (A) and strange (S) components. The fraction of $\pi^{-} \pi^{0} \omega$ in the $2 h^{-} h^{+} 2 \pi^{0}$ final state is assumed to be $(80 \pm 12) \%[12]$. For the $\tau$ decays fractions into $\pi \omega$ and $\pi \pi^{0} \omega$, the second error is associated to the $\omega$ fraction, which however does not contribute to the final uncertainties in the $V$ and $A$ components. The sum of the hadronic branching ratios in this table and the leptonic fractions from the global analysis is equal to $100 \%$. 
decays which yields $R_{\tau, V}=R_{\tau, A}$. More precisely, the measurements (9) and (10) imply in relative terms

$$
\frac{R_{\tau, V}-R_{\tau, A}}{R_{\tau, V}+R_{\tau, A}}=(2.7 \pm 1.3) \%
$$

in good agreement with an estimate of $(3.1 \pm 1.3) \%$ [37], based on non-perturbative corrections extracted from $e^{+} e^{-}$data. 

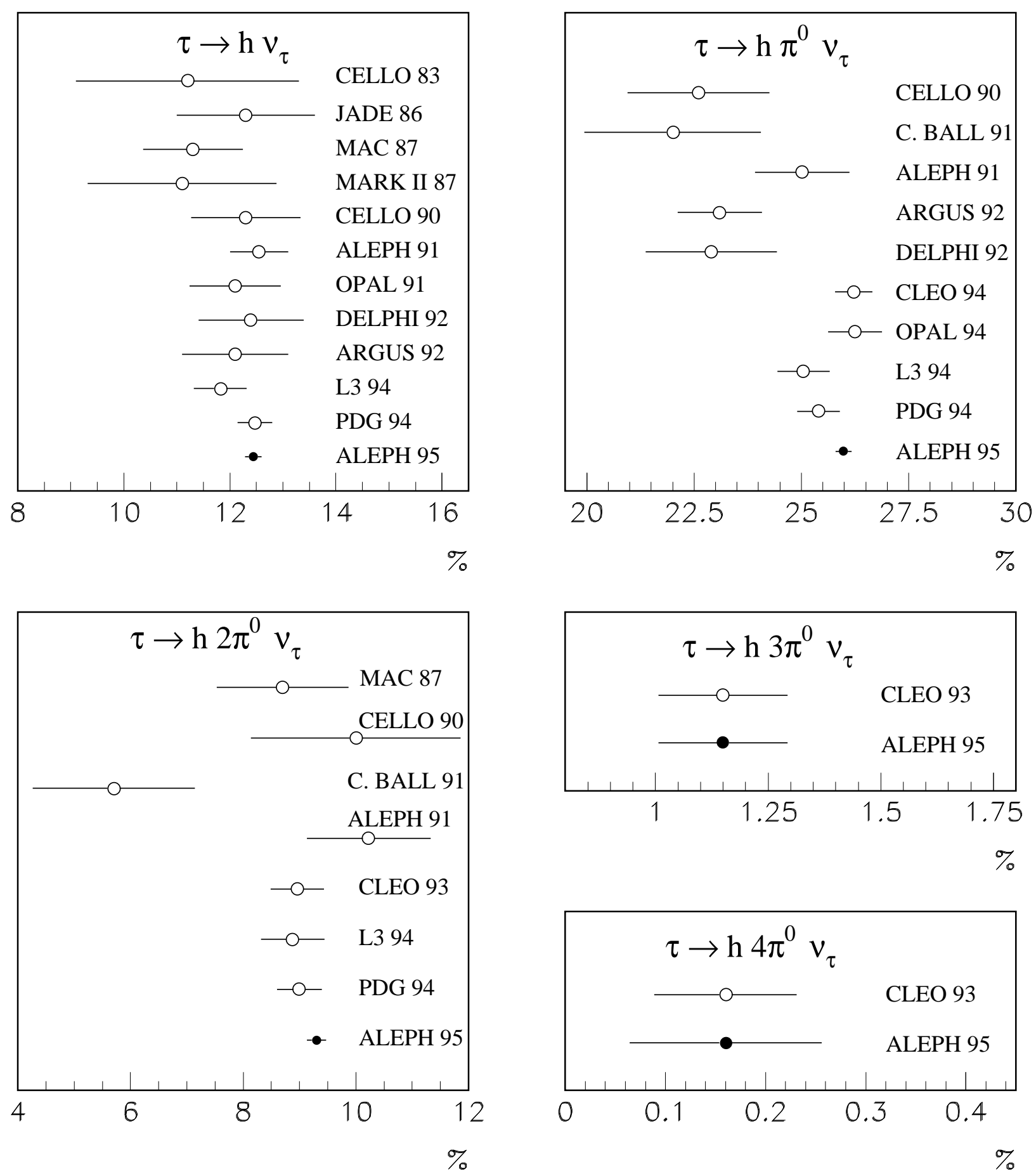

Figure 28: The published branching ratios of $\tau \rightarrow h \nu_{\tau}, \tau \rightarrow h \pi^{0} \nu_{\tau}, \tau \rightarrow h 2 \pi^{0} \nu_{\tau}$, $\tau \rightarrow h 3 \pi^{0} \nu_{\tau}$ and $\tau \rightarrow h 4 \pi^{0} \nu_{\tau}$ are shown [27]. The $\tau \rightarrow h \nu_{\tau}$ and $\tau \rightarrow h \pi^{0} \nu_{\tau}$ measurements contain the $K$ and $K_{L}^{0}$ contributions although probably not in a consistent way in different experiments. The $K_{S}^{0}$ contributions are subtracted in the $\tau \rightarrow h 2 \pi^{0} \nu_{\tau}, \tau \rightarrow h 3 \pi^{0} \nu_{\tau}$ and $\tau \rightarrow h 4 \pi^{0} \nu_{\tau}$ decay rates. 

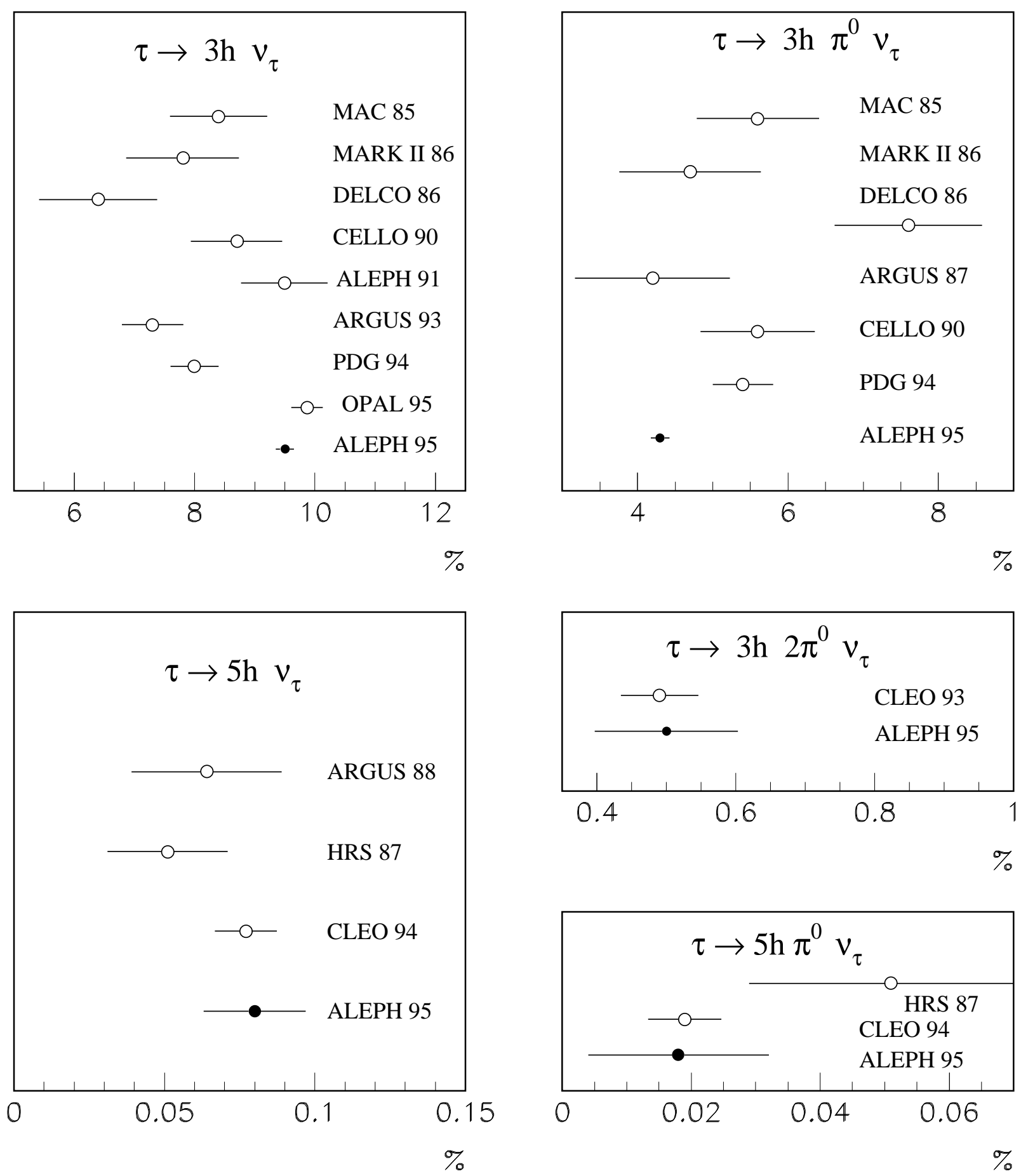

Figure 29: The published branching ratios of $\tau \rightarrow 3 h \nu_{\tau}, \tau \rightarrow 3 h \pi^{0} \nu_{\tau}, \tau \rightarrow 3 h 2 \pi^{0} \nu_{\tau}$, $\tau \rightarrow 5 h \nu_{\tau}$ and $\tau \rightarrow 5 h \pi^{0} \nu_{\tau}$ are plotted [27]. In all the channels the $K_{S}^{0}$ contributions are, in principle, subtracted out. 

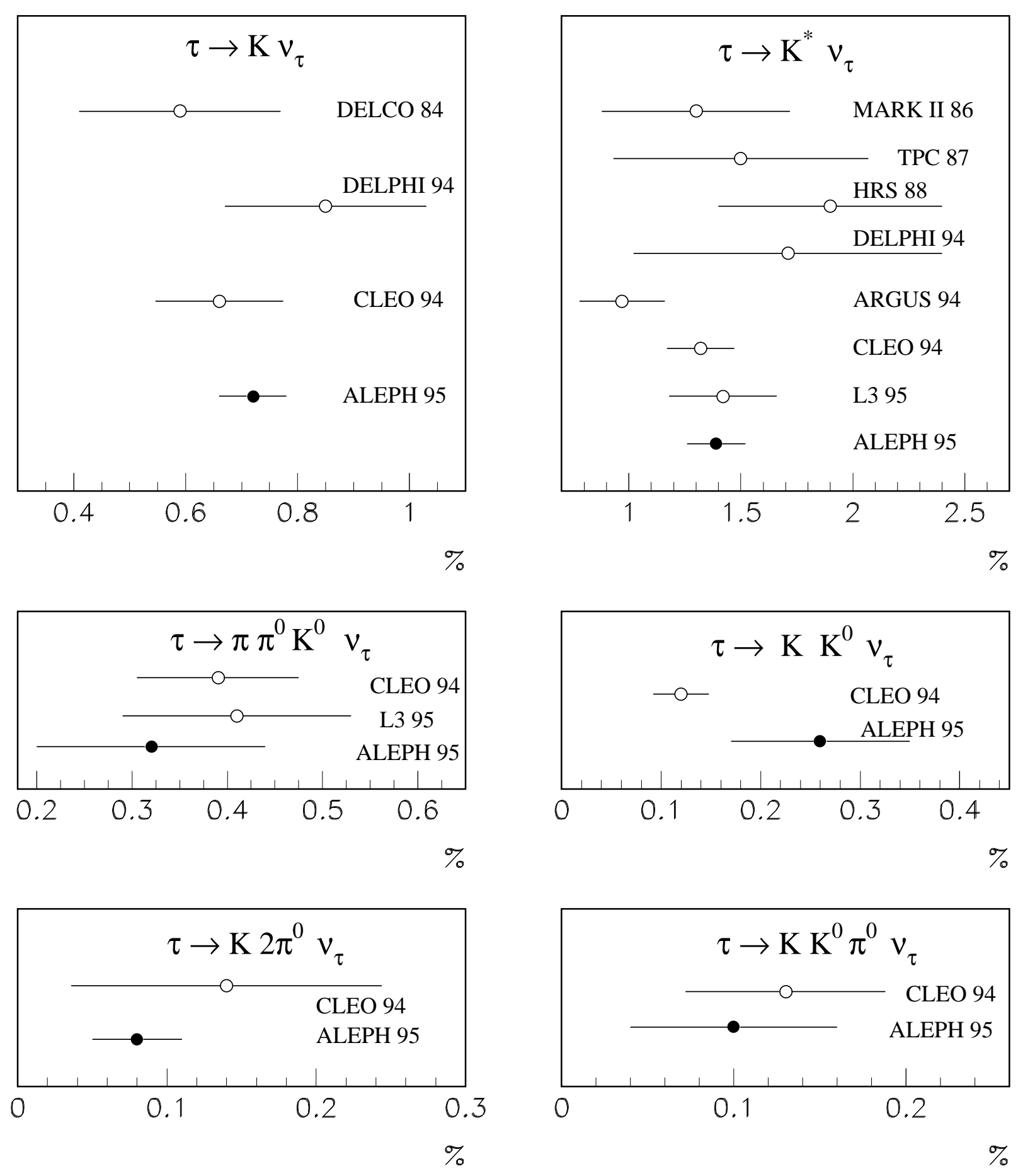

Figure 30: The published kaon branching ratios of $\tau \rightarrow K \nu_{\tau}, \tau \rightarrow K^{*} \nu_{\tau}, \tau \rightarrow \pi \pi^{0} K^{0} \nu_{\tau}$, $\tau \rightarrow K K^{0} \nu_{\tau}, \tau \rightarrow K 2 \pi^{0} \nu_{\tau}$ and $\tau \rightarrow K \pi^{0} K^{0} \nu_{\tau}$ are shown [27, 44, 45, 46, 47]. 


\section{Conclusions}

With the data collected by the ALEPH detector for 1991, 92 and 93 runs at $Z^{0}$ peak energies, $\tau$ pair events are selected with an efficiency of $78.8 \%$ and a background of $0.8 \%$. An overall $\pi^{0}$ efficiency of $83.7 \%$ is achieved over the whole energy range in the multi-hadronic environment of $\tau$ decays. A considerable effort is given to the problem of identifying fake photons in order to obtain a reliable $\tau$ decay classification. The measurement of the $\tau$ hadronic branching ratios is derived from a global analysis of $\tau$ decays. Quasi-exclusive and exclusive $\tau$ decays classifications are used to derive a complete and coherent set of $\tau$ hadronic branching ratios. The two classifications yield consistent results. The $\tau$ branching ratios into $h, h \pi^{0}, h 2 \pi^{0}$, $3 h$ and $3 h \pi^{0}$ are significantly more precise than the previous world averages.

\begin{tabular}{|l|c|c|}
\hline Mode & ALEPH & Standard Model \\
\hline \hline$B\left(\tau \rightarrow \pi^{-} \nu_{\tau}\right)$ & $11.06 \pm 0.18$ & $11.02 \pm 0.06$ \\
\hline$B\left(\tau \rightarrow K^{-} \nu_{\tau}\right)$ & $0.72 \pm 0.06$ & $0.73 \pm 0.01$ \\
\hline$B\left(\tau \rightarrow \pi^{-} \pi^{0} \nu_{\tau}\right)$ & $25.30 \pm 0.20$ & $24.6 \pm 0.7$ \\
\hline$B\left(\tau \rightarrow\left(a_{1}^{-} \rightarrow \pi^{-} 2 \pi^{0}\right) \nu_{\tau}\right)$ & $9.21 \pm 0.17$ & $9.03 \pm 0.43$ \\
\hline$B\left(\tau \rightarrow\left(a_{1}^{-} \rightarrow \pi^{-} \pi^{+} \pi^{-}\right) \nu_{\tau}\right)$ & $8.90 \pm 0.20$ & $9.03 \pm 0.43$ \\
\hline$B\left(\tau \rightarrow h^{-} 3 \pi^{0} \nu_{\tau}\right)$ & $1.17 \pm 0.14$ & $1.07 \pm 0.05$ \\
\hline$B\left(\tau \rightarrow 2 h^{-} h^{+} \pi^{0} \nu_{\tau}\right)$ & $4.30 \pm 0.12$ & $4.20 \pm 0.30$ \\
\hline$B\left(\tau \rightarrow(6 \pi)^{-} \nu_{\tau}\right)$ & $0.13 \pm 0.06$ & $0.13 \pm 0.02$ \\
\hline
\end{tabular}

Table 17: The measured branching ratios of $\tau \rightarrow \pi^{-} \nu_{\tau}, \tau \rightarrow K^{-} \nu_{\tau}, \tau \rightarrow \pi^{-} \pi^{0} \nu_{\tau}$, $\tau \rightarrow \pi^{-} 2 \pi^{0} \nu_{\tau}, \tau \rightarrow \pi^{-} \pi^{+} \pi^{-} \nu_{\tau}, \tau \rightarrow h^{-} 3 \pi^{0} \nu_{\tau}, \tau \rightarrow 2 h^{-} h^{+} \pi^{0} \nu_{\tau}$, and $\tau \rightarrow(6 \pi)^{-} \nu_{\tau}$ are shown with the expected values from the Standard Model as discussed in Section 13.

The branching ratios presented here are consistent with the Standard Model as given in Table 17.

Using the measurements on $\tau \rightarrow \pi \nu_{\tau}$ and $\tau \rightarrow K \nu_{\tau}$, the $\mu-\tau$ universality of the weak coupling in the charged current is tested with the result

$$
\frac{g_{\tau}}{g_{\mu}}=1.0013 \pm 0.0095
$$

Finally, a separation of the vector and axial hadronic contributions is performed for the 
first time. The ratio

$$
\frac{R_{\tau, V}-R_{\tau, A}}{R_{\tau, V}+R_{\tau, A}}=(2.7 \pm 1.3) \%
$$

is consistent with estimates of non-perturbative corrections in the $\tau$ hadronic decay width. Therefore, these results support the perturbative QCD description of hadronic $\tau$ decays.

\section{Acknowledgements}

We wish to thank our colleagues from the accelerator divisions for the successful operation of LEP. We are indebted to the engineers and technicians in all our institutions for their contribution to the excellent performance of ALEPH. Those of us from non-member countries thank CERN for its hospitality. 


\section{References}

[1] Proceedings of the third Workshop on Tau Lepton Physics, Montreux 1994, L. Rolandi ed., Nucl. Phys. (Proc. Suppl.) B40 (1995) .

[2] CELlo Collaboration, H.J. Behrend et al., Zeit. für Physik C46 (1990) 537.

[3] ALEPH Collaboration, D. Decamp et al., Zeit. für Physik C54 (1992) 211.

[4] ALEPH Collaboration, 'Tau leptonic branching ratios', D. Buskulic et al., CERN-PPE/95-127.

[5] ALEPH Collaboration, D. Buskulic et al., Phys. Lett. B332 (1994) 209.

[6] ALEPH Collaboration, D. Buskulic et al., Phys. Lett. B332 (1994) 219.

[7] ALEPH Collaboration, D. Decamp et al., Nucl. Instr. Methods A294 (1990) 127.

[8] ALEPH Collaboration, D. Buskulic et al., Nucl. Instr. Methods A360 (1995) 481.

[9] S. Jadach, B.F.L. Ward, and Z. Was, Comp. Phys. Comm. 79 (1994) 503.

[10] S. Jadach et al., Comp. Phys. Comm. 76 (1993) 361.

[11] ALEPH Collaboration, D. Decamp et al., Zeit. für Physik C62 (1994) 539.

[12] CLEO Collaboration, D. Bortoletto et al., Phys. Rev. Lett. 71 (1993) 1791.

[13] CLEO Collaboration, M. Artuso et al., Phys. Rev. Lett. 69 (1992) 3278.

[14] DELCO Collaboration, G. Mills et al., Phys. Rev. Lett. 54 (1985) 624.

[15] TPC/Two-Gamma Collaboration, D. Bauer et al., Phys. Rev. D50 (1994) R13.

[16] R. Decker et al., Zeit. für Physik C58 (1993) 445.

[17] P. Bourdon, (ALEPH Collaboration), Proceedings of the third Workshop on Tau Lepton Physics, Montreux 1994, L. Rolandi ed., Nucl. Phys. (Proc. Suppl.) B40 (1995) .

[18] S. Snow, Proceedings of the 2nd International Workshop on $\tau$ Lepton Physics, Columbus 1992, K.K. Gan ed., World Scientific (1993).

[19] ALEPH Collaboration, D. Buskulic et al., Zeit. für Physik C59 (1993) 369.

[20] N. Isgur et al., Phys. Rev. D39 (1989) 1357.

[21] ARGUS Collaboration, H. Albrecht et al., Zeit. für Physik C58 (1993) 61.

[22] OPAL Collaboration, R. Akers et al., CERN-PPE/95-22. 
[23] Z. Was, private communication.

[24] R. Decker and M. Finkemeier, Phys. Rev. D48 (1993) 4203.

[25] W.J. Marciano and A. Sirlin, Phys. Rev. Lett. 61 (1988) 1815.

[26] ALEPH Collaboration, D. Buskulic et al., CERN-PPE/95-128.

[27] Particle Data Group, L. Montanet et al., Phys. Rev. D50 (1994) 1173.

[28] CLEO Collaboration, M. Artuso et al., Phys. Rev. Lett. 72 (1994) 3762.

[29] OPAL Collaboration, R. Akers et al., CERN-PPE/94-42.

[30] L3 Collaboration, M. Acciarri et al., CERN-PPE/94-178.

[31] S. Eidelman et al, Proceedings of the third Workshop on Tau Lepton Physics, Montreux 1994, L. Rolandi ed., Nucl. Phys. (Proc. Suppl.) B40 (1995) .

[32] T. Das, V. Mathur and S. Okubo, Phys. Rev. Lett. 18 (1967) 761.

[33] E. Floratos, S. Narison and E. De Rafael, Nucl. Phys. B155 (1979) .

[34] OPAL Collaboration, R. Akers et al., CERN-PPE/95-70.

[35] M.Davier, Proceedings of the 2nd International Workshop on $\tau$ Lepton Physics, Columbus 1992, K.K. Gan ed., World Scientific (1993).

[36] S. Narison and A. Pich, Phys. Lett. B304 (1993) 359.

[37] E. Braaten, S. Narison and A. Pich, Nucl. Phys. B373 (1992) 581.

[38] CLEO Collaboration, M. Procario et al., Phys. Rev. Lett. 70 (1993) 1207.

[39] CLEO Collaboration, D. Gibaut et al., Phys. Rev. Lett. 73 (1994) 934.

[40] T.N. Pham, C. Roiesnel, and T.N. Truong, Phys. Lett. B78 (1978) 623.

[41] CLEO Collaboration, M. Battle et al., Phys. Rev. Lett. 73 (1994) 1079.

[42] L3 Collaboration, M. Acciarri et al., CERN-PPE/95-42.

[43] T. Pich, F. Le Diberder, Proceedings of the QCD Workshop, Montpellier 1994, S. Narison ed., Nucl. Phys. (Proc. Suppl.) B39 (1995).

[44] DELPHI Collaboration, P. Abreu et al., CERN-PPE/94-88.

[45] CLEO Collaboration, M. Athanas et al., CLEO CONF 94-23, Submitted to Int. Conf. on High Energy Physics, Glasgow, Scotland, July, 1994. 
[46] CLEO Collaboration, J. Gronberg et al., CLEO CONF 94-24, Submitted to Int. Conf. on High Energy Physics, Glasgow, Scotland, July, 1994.

[47] CLEO Collaboration, R. Balest et al., CLEO CONF 94-25, Submitted to Int. Conf. on High Energy Physics, Glasgow, Scotland, July, 1994. 